The influence of brain-skull interactions in the development of the amphibian skull

$$
\text { By }
$$

\title{
Erin MacKenzie
}

A thesis submitted to the Faculty of Graduate and Postdoctoral Affairs in partial fulfillment of the requirements for the degree of

\author{
Master of Science
}

in

Earth Sciences

Carleton University

Ottawa, Ontario

(C) 2021, Erin MacKenzie 


\section{$\underline{\text { Abstract }}$}

The tetrapod skull has evolved in response to various pressures over its long evolutionary history. One such pressure is the expansion of the cerebral hemispheres of the brain, which has been shown to drive physical changes in the craniofacial skeleton in certain lineages. The molecular basis of these brain-skull interactions remains incompletely understood, though studies indicate that key to this relationship is the range of morphogenetic signals originating from signaling sources within the forebrain. Knockdown experiments in mouse and chick have been conducted to eliminate these signals and study the resulting morphology; however, interrupting gene expression may have off-target effects. An alternative method of eliminating local signals is tissue removal/ablation. To better understand the role of the forebrain in shaping cranial development, forebrain removal experiments were conducted in Xenopus laevis, a representative of the poorly studied amphibians, to study the resulting morphology of the skull. To facilitate these comparisons, a detailed internal staging guide for $X$. laevis was developed, expanding on the widely used Nieuwkoop and Faber series. Next, it was found that removal of forebrain tissue in early tailbud embryos resulted in craniofacial deformities, as well as a disruption of olfactory and optic sensory development. Potential biochemical causes of these results are discussed within the context of knowledge from amniote systems. These findings indicate that the role of the forebrain in craniofacial development seen in amniotes is likely conserved in X. laevis, providing vital information for our understanding of tetrapod skull evolution. 


\section{$\underline{\text { Acknowledgements }}$}

I would like to thank several people who have helped me with support and assistance throughout this thesis. Firstly, I would like to thank my supervisor, Dr. Hillary Maddin, for her encouragement and feedback in a field I had previously unexplored. Challenging me to understand how to apply my embryological knowledge to the broader, evolutionary aspect challenged my way of thinking about the bigger picture experiments. Secondly, I would like to thank Dr. Iain McKinnell for always taking time to brainstorm and bat some ideas around with me over a coffee/tea. His encouragement and support in my technical skills has helped greatly solidify my confidence as a scientist.

I would also like to thank the members of the Maddin Lab for their collaboration and aid. Specifically, I would like to thank Jade Atkins for her invaluable help. Her help as a colleague and as a friend was an integral part of the completion of my thesis. I would also like to thank Dana Korneisel for many things, including her Adobe Illustrator expertise and Alanna Cantelon for her dedication to the animal welfare and care.

I would like to thank my partner, Tryston Charlton, for his incredible emotional support throughout this time. As I tend to get carried away and overwork myself to the point of burnout, he always made sure to check in on me, encourage breaks, bring me a snack/coffee, and prioritize my own mental health over academic strain. I would like to thank my family and friends for putting up with me while the only thing I talked about was my thesis.

Thanks to Happy Goat Coffee for fueling my writing sessions. Finally, I would like to not thank COVID-19 and OC-Transpo, both of which provided major interruptions in my busy schedule. 


\section{$\underline{\text { Table of Contents }}$}

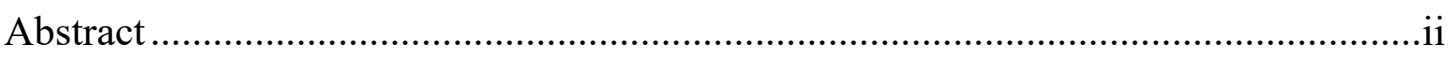

Acknowledgements........................................................................................... ii

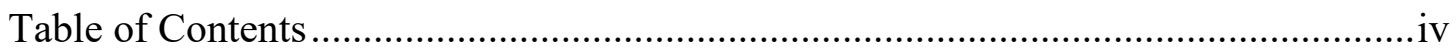

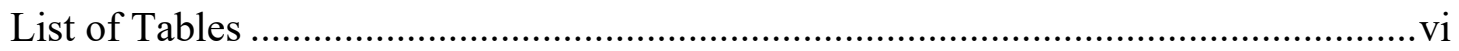

List of Figures ................................................................................................

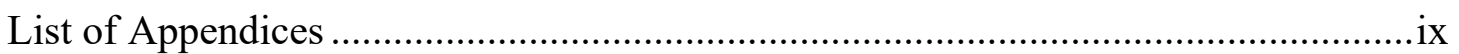

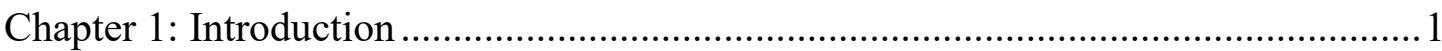

1.1: Modifications to the vertebrate skull over time …………………………………….... 1

1.2: Developmental basis for craniofacial deformities .....................................................

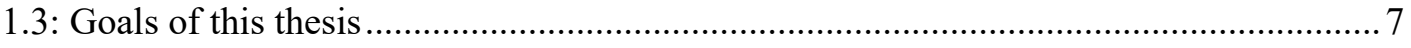

Chapter 2: Normal skull development in Xenopus laevis - a complement to the

Nieuwkoop and Faber staging series ……………………................................ 12

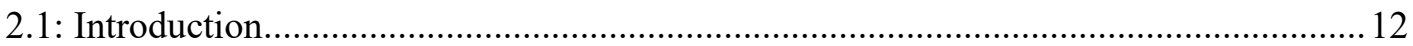

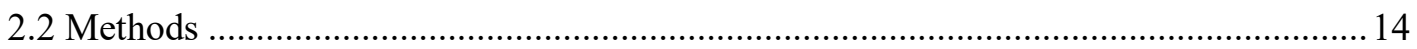

2.2.1: Animal Care and Breeding............................................................................. 14

2.2.2: Staging and Fixation .......................................................................................... 15

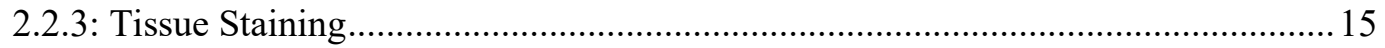

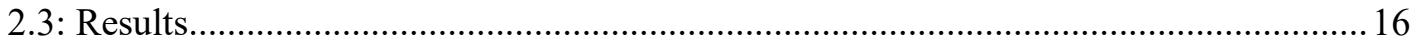

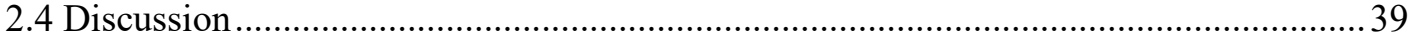

Chapter 3: Surgical manipulation of Xenopus laevis embryonic forebrain to study its role in the structural development of the amphibian cranium ......................................45

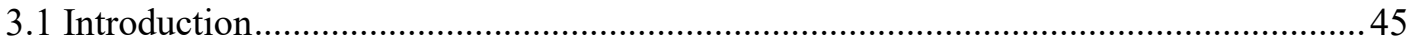

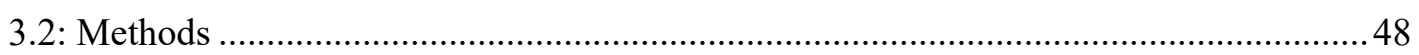

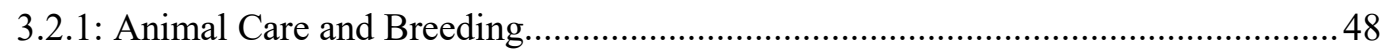

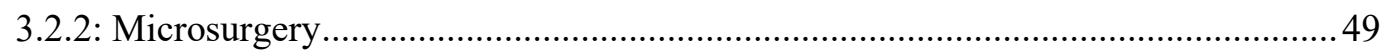

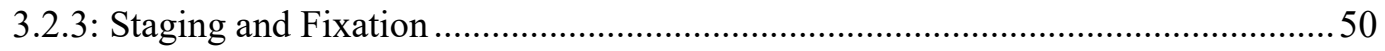

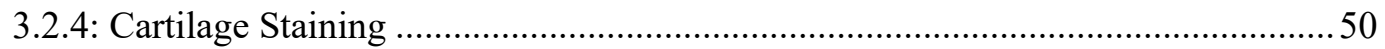




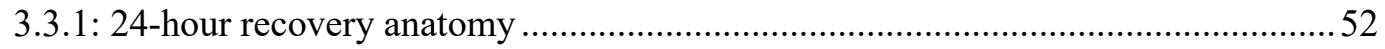

3.3.2: External features post-surgery …………....................................................... 53

3.3.3: Effects of forebrain removal on chondrocranial development...............................56

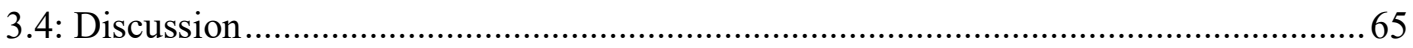

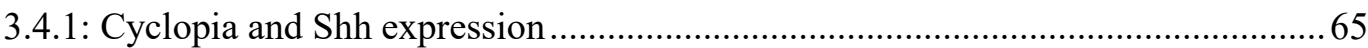

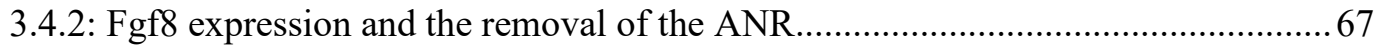

3.4.3: Optic development interrupted with the removal of the forebrain......................... 70

3.4.4: Olfactory development interrupted with the removal of the forebrain ...................72

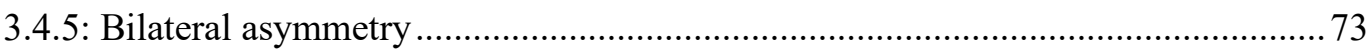

3.4.6: Forebrain removal does not disrupt the posterior elements of the skull ................ 76

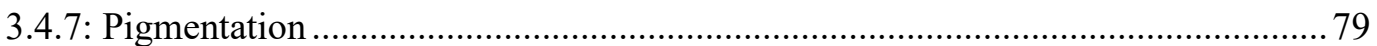

3.4.8: Removal of forebrain reduced viability of experimental specimens ..................... 79

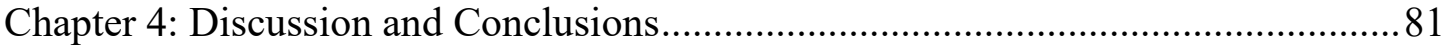

4.1: Changes to the chondrocranium via forebrain removal can interfere with chondro-

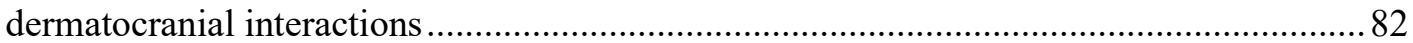

4.2: The forebrain is required for normal optic and olfactory sensory development........... 84

4.3: Development of the chondrocranium is anteroposteriorly regionalized by instructions

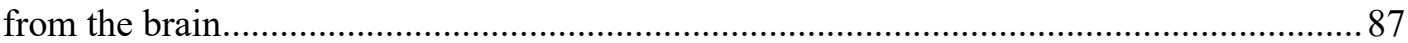

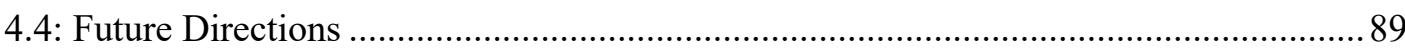

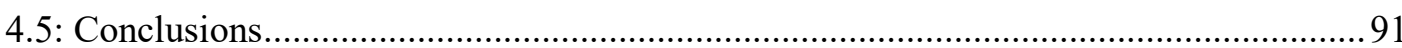

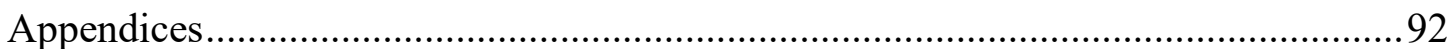

Appendix A: Xenopus laevis staging series specimen numbers and sequence of ossification .92

Appendix B: Xenopus laevis $\mathrm{FB}^{-}$specimen numbers, stage, and methods schematic.........94

References 95 


\section{$\underline{\text { List of Tables }}$}

Table A1: Number of Xenopus laevis specimens included in the staging series, cartilage and bone stain. 92

Table A2: Cranial ossification sequence in Xenopus laevis .93

Table B1: Xenopus laevis $\mathrm{FB}^{-}$specimen numbers and stage of surgery 94 


\section{List of Figures}

Figure 1.1: Skull endocasts of archaic primates and early hominids show the evolution of the forebrain and the relative cranium capacity. 2

Figure 1.2: Cranial endocasts to illustrate the relationship between soft tissue and skull roof evolution in A. mississippiensis and G. gallus .5

Figure 1.3: Double knockdown of chordin and noggin results in holoprosencephaly .. 8

Figure 1.4: Expression profile of $S h h$ and $F g f 8$ in the anterior forebrain of an $\mathrm{HH}$ stage 20 chick embryo 9

Figure 2.1: NF stages 41 to 48, Xenopus laevis cartilaginous skull .17

Figure 2.2: NF stages 49 to 53, Xenopus laevis cartilaginous skull .24

Figure 2.3: NF stages 54 to 59, Xenopus laevis osseous skull. .26

Figure 2.4: NF stages 60 to 63, Xenopus laevis osseous skull. .31

Figure 2.5: NF stages 64 to 66, Xenopus laevis osseous skull. .36

Figure 3.1: Examples of Xenopus laevis embryo features post-forebrain surgery 53

Figure 3.2: External/internal features and gross morphology of Xenopus laevis postsurgery $\mathrm{FB}^{-}$specimens .55

Figure 3.3: Palatal and rostral development of Xenopus laevis post-surgery $\mathrm{FB}^{-}$ specimens .58

Figure 3.4: Mandibular development of Xenopus laevis post-surgery $\mathrm{FB}^{-}$specimens 60

Figure 3.5: Development of the hyoid arch in Xenopus laevis post-surgery $\mathrm{FB}^{-}$ specimens 
Figure 3.6: Development of the subocular structures in Xenopus laevis post-surgery $\mathrm{FB}^{-}$specimens... .64

Figure 3.7: Development of the ventrolateral and ascending processes of the palatoquadrate in Xenopus laevis post-surgery $\mathrm{FB}^{-}$specimens..... .65

Figure B1: Methodological schematic of forebrain removal surgery on Xenopus laevis tailbud embryos.. .94 


\section{List of Appendices}

Appendix A: Xenopus laevis staging series specimen numbers and sequence of

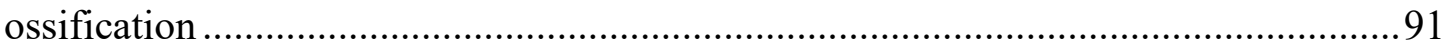

Appendix B: Xenopus laevis $\mathrm{FB}^{-}$specimen numbers stage and methods schematic ..93 


\section{Chapter 1: Introduction}

The tetrapod skull is a vastly diverse structure that is the manifestation of complex interacting factors including phylogeny, function, and development. As the head is an intricate collection of many tissues and cell types, these parts must develop together with strict cohesion to ensure proper development and tissue integration. It is known that in amniotes (specifically mammals and birds), expansion of the brain has influenced skull shape evolution wherein frontal and parietal bone expansion is correlated with cerebral hemisphere expansion (Morriss-Kay, 2001; Maddin et al., 2016). Most studies that investigate the correlations between brain and skull evolution focus on the available fossil record for comparative analyses (Schoch, 2014; Blackburn et al., 2019), whereas others have discussed the mechanical pressures of encephalization (Jiang et al., 2002; Lesciotto and Richtsmeier, 2019). Few, on the other hand, examine the biochemical processes that underlie the relationship between the brain and skull, and so the molecular basis for this causation has been discussed but as of yet, remains incompletely understood.

\section{1: Modifications to the vertebrate skull over time}

Evolutionarily, the tetrapod skull has undergone many significant alterations such as cranial base flexion, variation in temporal fenestration, addition and loss of cranial elements, and sutural variation (Markey and Marshall, 2007; Lesciotto and Richtsmeier, 2019; Werneberg, 2019). Determining the origin of these anatomical modifications can help us gain insights into critical events that explain the story of tetrapod skull evolution. 


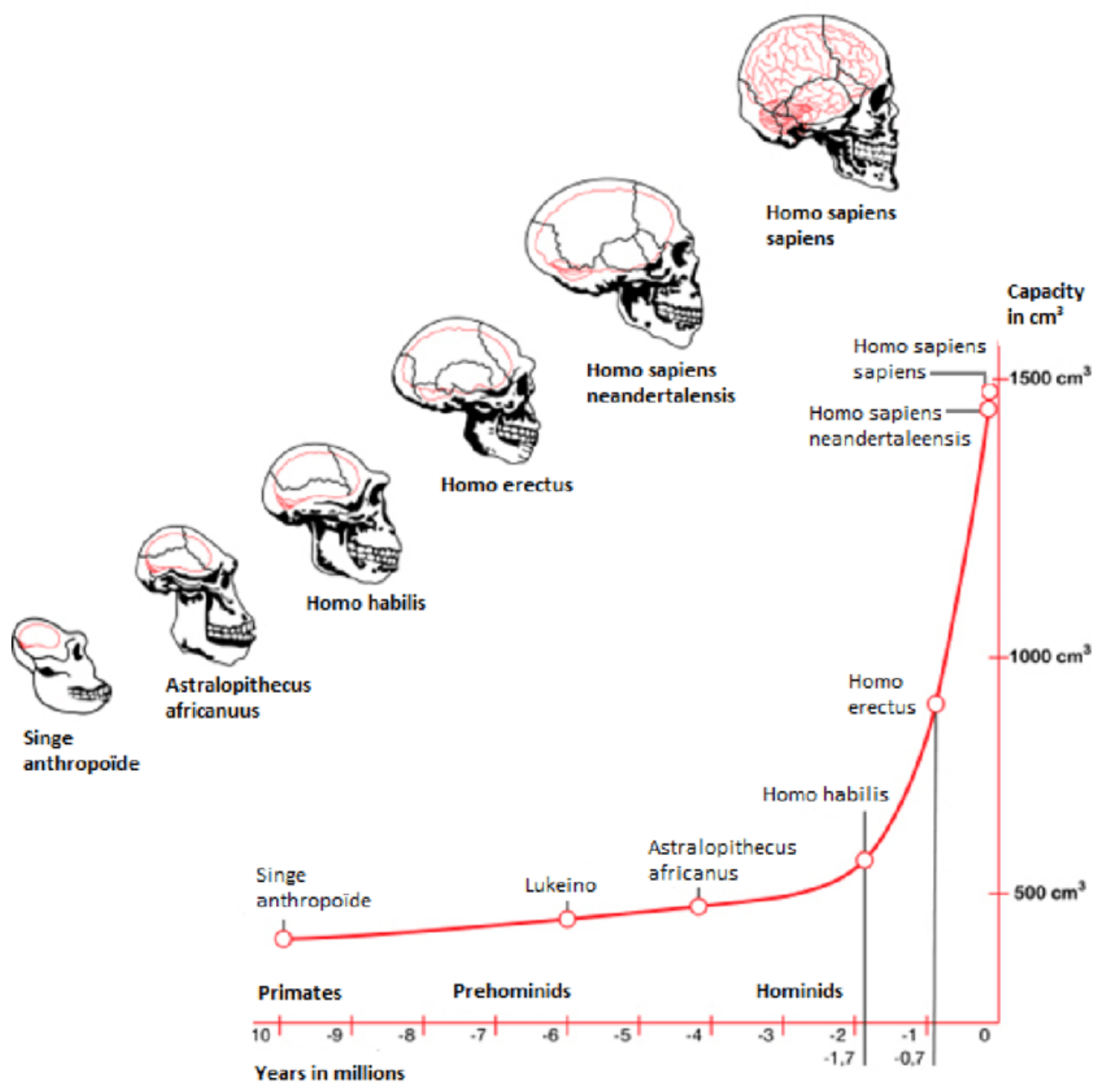

Figure 1.1. Skull endocasts of archaic primates and early hominids show the evolution of the forebrain and the relative cranium capacity. From van Ginneken et al., 2017).

Early insights into a potential evolutionary correlation between the brain and skull came from paleontological studies of the cranial endocasts of fossil taxa. There, fossil skulls were filled with latex to create $3 \mathrm{D}$, physical endocasts of the brain cavity (Falk, 1987). The endocasts of hominids were thoroughly examined in the 1960s, where it was demonstrated that expansion of the brain correlated with expansion of the overlying skull bones (Fig 1.1). Few contemporaneous studies, however, 
investigated non-hominid species, except for the trail-blazing study of Romer and Edinger (1942). Romer and Edinger (1942) studied the endocasts of both extinct and extant amphibian species to better understand the structure of the ancestral amphibian nervous system. They found that the skull bones overlying the telencephalon were much thinner due to expansion during development, as well as having a very detailed internal surface, indicating significant contouring of the skull to the brain surface. Since this early study, more advanced technologies have been developed that allow endocasts to be created digitally using computed tomography (CT) (e.g., Balanoff et al., 2016). This non-destructive method has made available an abundance of fossil specimens, which has yielded numerous new studies that support a more widespread pattern of correlation between the brain and skull in amniote tetrapods (Morriss-Kay, 2001; Jiang et al., 2002; Noden and Trainor, 2005; McBratney-Owen et al., 2008; Maddin et al., 2016; Fabbri et al., 2017 [Fig. 1.2]). Amphibians remain relatively poorly studied in this regard, however.

Encephalization is the process in which the size of the brain increases relative to the size of the body (Lesciotto and Richtsmeier, 2019). As the brain evolved and increased in size in certain lineages, the bones surrounding the brain needed to adjust to continue protecting it - the most conspicuous of which comprise the craniofacial skeleton (Kuratani et al., 2016). The tissues that give rise to these structural elements of the face and skull roof are of cranial neural crest $(\mathrm{CNC})$ origin, which separates from the neural tube upon closure, and migrates and differentiates into chondrocytes and osteoblasts (Anderson, 1997). A key factor in chondrocyte differentiation is FGF8, a morphogen that plays a significant role in neural patterning (Abzhanov and Tabin, 2004; Creuzet et al., 2004). This morphogen is highly concentrated in two areas of the developing brain - the anterior neural ridge (ANR) and the 
midbrain/hindbrain boundary (Vieira et al., 2010). The ANR is located at the anteriormost point of the embryonic brain in the forebrain region. FGF8 signals from the ANR have been shown to be necessary to promote CNC cell survival and differentiation, which is a requirement for subsequent development of craniofacial structures (Abzhanov and Tabin, 2004).

This thesis focuses on the influence of the forebrain on cranial development. This region of the brain is emphasized specifically because it has undergone the most evolutionary modifications in terms of shape and size compared to the midbrain and hindbrain (Hofman, 2014). As the cerebral hemispheres expanded, so too did the frontal and parietal bones, as they were required to respond to encephalization (Jiang et al., 2002; Lesciotto and Richtsmeier, 2019). Thus, if the growth of the brain is driving structural change on a larger, evolutionary timescale, then examining the interactions between the brain and bone on a smaller, developmental timescale has the potential to reveal key insights about this relationship. This was studied here in amphibians, specifically Xenopus laevis, for several reasons. Firstly, we currently lack data concerning a potential correlation between brain and skull development in amphibians. Secondly, if found to be the case, those data would reveal a deeper conservation of brain-skull interactions exists in tetrapods. Lastly, the shared patterns of these interactions will allow us to potentially apply data from amphibians to latediverging vertebrates, such as reptiles, aves, and mammals, which can be more challenging to work with in the lab. Anurans also have a unique cranial feature, the frontoparietal, which is considered to be a fusion of an ancestrally distinct frontal and parietal. I will investigate outstanding questions surrounding the anuran frontoparietal in terms of how the brain may interact with and influence this distinctive structure 

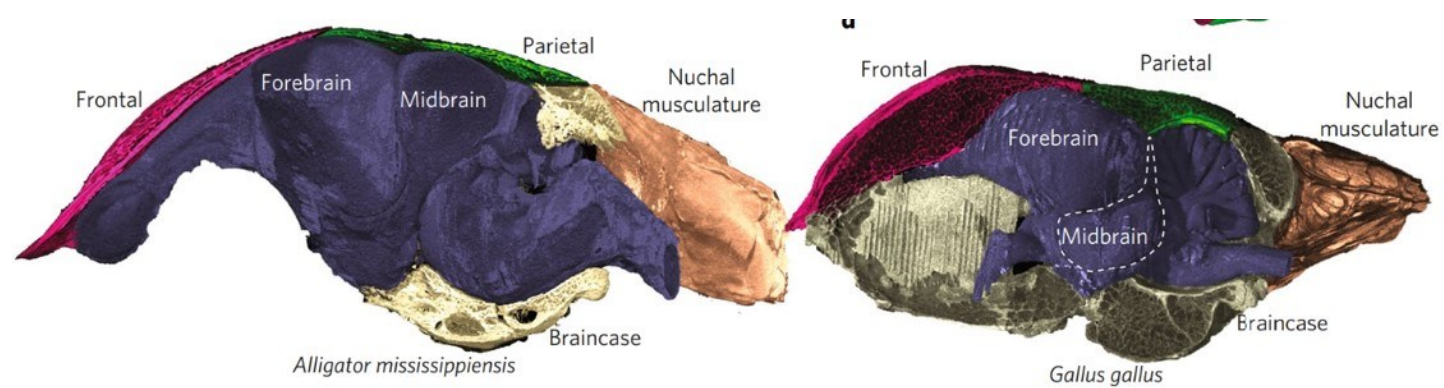

Figure 1.2. Digital models of portions of the skull vault and cranial endocasts (in purple) to illustrate the relationship between the brain and skull roof evolution in Alligator mississippiensis and Gallus gallus. Skull is hemisectioned in the parasagittal plane, with anterior to the left. The forebrain can be seen to reside adjacent to the frontal bone (pink) of the skull, whereas the midbrain and hindbrain reside adjacent to the parietal bone (green). From Fabbri et al., 2017.

(Roček, 1988). Some research has suggested that the development of the fore- and midbrain relates to the frontal and parietal bones in Reptilia and Aves, respectively (Fabbri et al., 2017). In studying this relationship in $X$. laevis, we can trace back this proposed relationship to see how early it can be observed among tetrapods.

\section{2: Developmental basis for craniofacial deformities}

Much of what we have learned about the developmental correlation between the brain and skull has been derived from clinical research. Over many decades, documented developmental disorders in human have revealed how deformations of the brain lead to a variety of craniofacial malformations, impacting skull morphology. The defined elements of the craniofacial skeleton vary across species but generally consist of palatal, nasal, maxillary, and mandibular structures, as well as the frontal bone (Richtemeier and Flaherty, 2013; Dubey and Saint-Jeannet, 2017). Neurocristopathies are deformations of the craniofacial skeleton that specifically arise from abnormal development of cranial neural crest $(\mathrm{CNC})$ cells. The resulting malformations can arise in CNC cells that are either fated to become neurons or 
chondrocytes/osteoblasts, meaning that the origin of this anomaly can be in both presumptive brain and bone tissues (Müller and O'Rahilly, 1991; Bolande, 1997; Roessler et al., 1997; Cohen, 2006; Raam et al., 2010; Shim et al., 2016; Vega-Lopez et al., 2018). CNC cells play a variety of important roles in the head as they give rise to connective tissues, bones, neurons, and smooth muscle (Dubey and Saint-Jeannet, 2017). With this knowledge, it becomes clear why the vast majority of craniofacial deformities are linked to abnormal $\mathrm{CNC}$ development. It has also been demonstrated that most craniofacial developmental patterns seem to be well conserved across vertebrate species, mainly in accordance with CNC patterns, which further establishes the role of $\mathrm{CNC}$ as a primary driving force in cranial evolution (Hanken and Gross, 2005; Cordero et al., 2011).

Although CNC cells give rise to certain cranial structures, they are not the only factor in coordinating skull development (Grenier et al., 2009). Much of normal craniofacial development is owed to the well-coordinated migration and displacement of various tissues in the head, such as closure or the neural tube leading to some epithelial cells adopting a mesenchymal fate as CNC cells (Helms et al., 2005). This leads us to believe that if these tissue relocation events were physically interrupted, such as with microsurgical manipulation or genetic interference, one could impact the resulting skull morphology and induce phenotypes similar to those derived from neurocristopathies. Additionally, multiple signaling centres within the developing brain are known for their role in coordinating cranial development via communication with the CNC cells that produce the skull structures (Kobayashi et al., 2002; Vieira et al., 2010; Schmidt et al., 2018). This suggests that the connection between the forebrain and skull patterning likely occurs via key signaling pathways and controlled gene expression. One such signaling pathway is Sonic hedgehog (Shh). When Shh is 
inhibited, the frontonasal prominence is not able to provide necessary signals (expression of Shh, Ptc, and Glil) to the surrounding tissue leading to a severe interruption of facial development (Marcucio et al., 2005). In chick and mouse, disrupting $S h h$ expression in the forebrain interferes with the signaling centre that controls outgrowth of the facial primordia - the Frontonasal Ectodermal Zone (FEZ). This results in malformed palatine and maxillary bones. While the existence of the FEZ is uncertain in Xenopus, its development in chick and mouse involves expression of Fgf8 and Shh, two genes that are critical for facial development in Xenopus (Chen et al., 2017). Further investigation of the role of the forebrain in Xenopus facial skeleton primordia patterning will provide valuable information for the connection between facial development in poorly-known early-diverging tetrapods. Furthermore, double knockout of Chordin and Noggin, two genes required for forebrain development, results in hindered forebrain development and holoprosencephaly (i.e., olfactory/optic cyclopia, agnathia) in mouse, which potentially interferes with signals emanating from the forebrain that coordinate CNC cell migration (Bachiller et al., 2000 [Fig. 1.3]; Abzhanov and Tabin, 2004 [Fig. 1.4]).

\section{3: Goals of this thesis}

The goal of this thesis is to examine the role that the forebrain plays in the development and morphology of the skull in an amphibian species, Xenopus laevis. The fossil record has revealed coordinated changes in the brain and skull in certain groups of tetrapods (i.e., hominids and birds); however, this research does not explain the mechanistic basis of such changes, nor have such studies been applied more broadly to non-amniote groups of tetrapods, such as amphibians. More recently, 

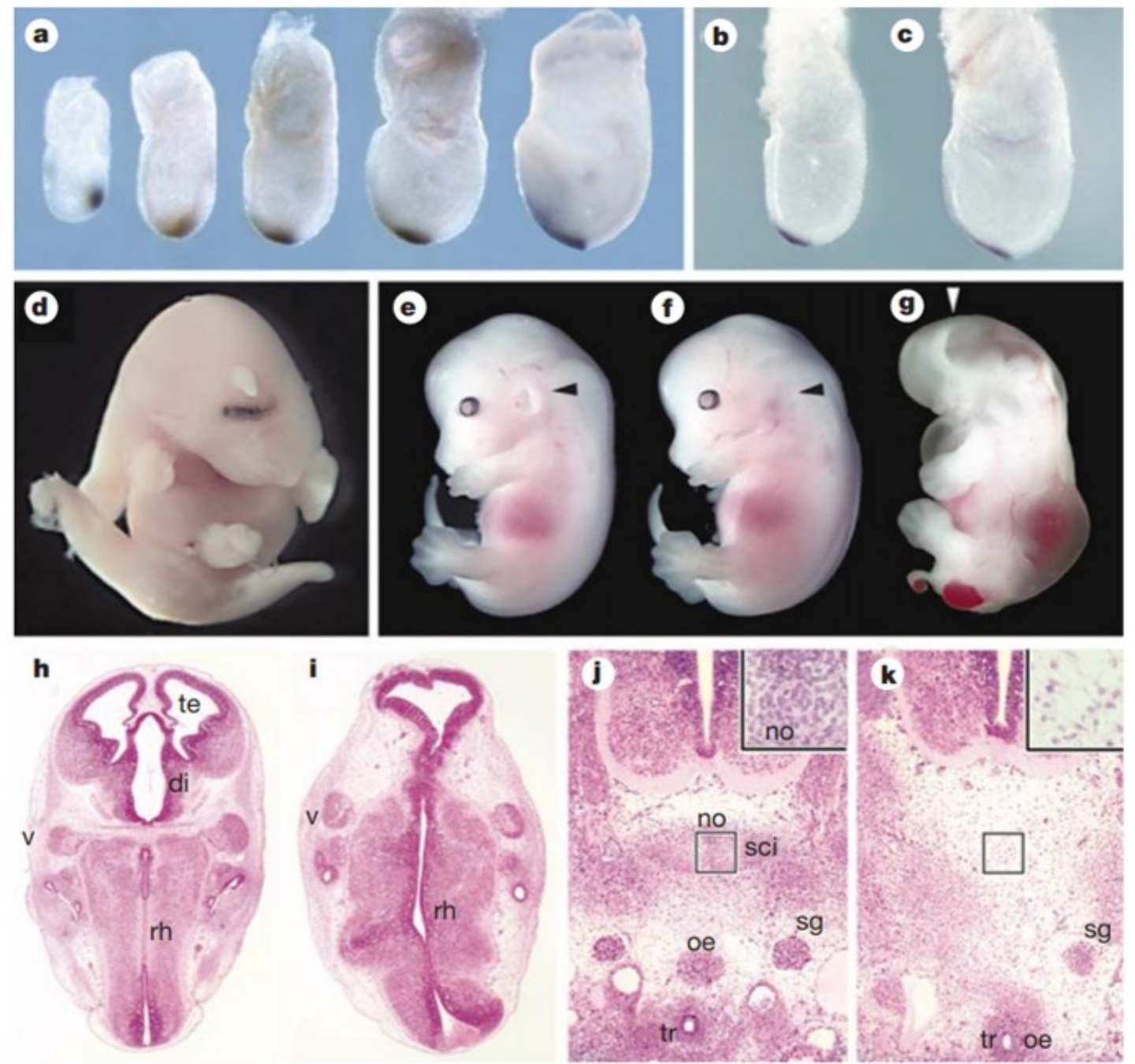

Figure 1.3. Double knockdown of chordin and noggin results in holoprosencephaly. A, Expression of chordin in a mouse embryo at gastrulation. B and $\mathrm{C}$, Expression of noggin in mouse embryo at gastrulation. D-G, Chd $\mathrm{d}^{-/ / \mathrm{Nog}^{-/}}$ embryos show optic cyclopia, single nasal pit, and agnathia. $\mathrm{H}$ and I, Coronal section of a wild-type embryo and $\mathrm{Chd}^{-/ /} / \mathrm{Nog}^{-/-}$embryo. The wild-type displays paired telencephalic vesicles and normal diencephalon, whereas double-knockout produces a single forebrain vesicle with telencephalon and diencephalon. From Bachiller et al., 2000. 


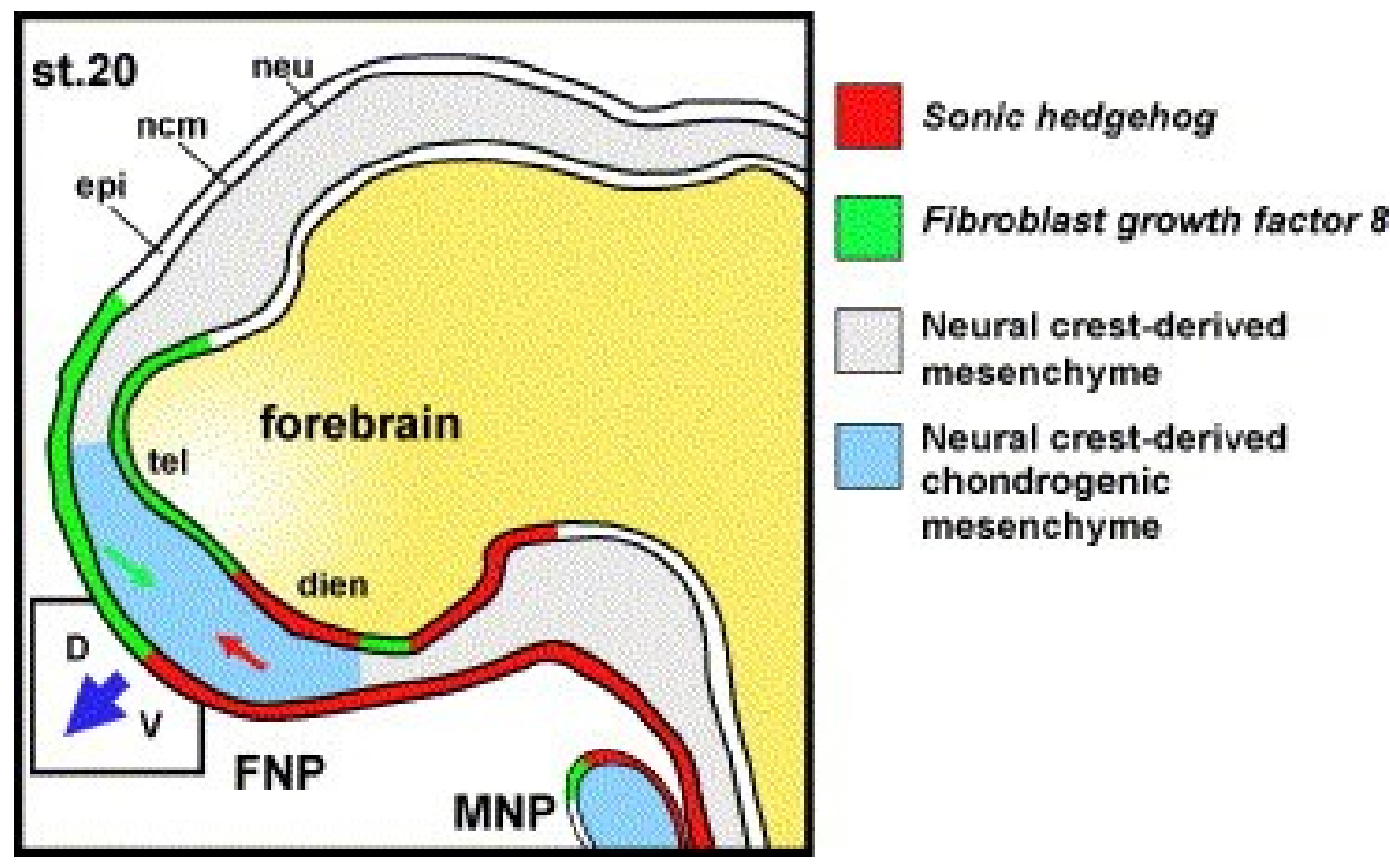

Figure 1.4. Expression profile of $S h h$ and $F g f 8$ in the anterior forebrain of an HH stage 20 chick embryo. $F g f 8$ expression is localized to the developing telencephalon, in the region of the anterior neural ridge. Shh expression is more ventral than $F g f 8$ expression, as it is produced in the ventral neural tube and notochord. From Abzhanov and Tabin, 2004. Abbreviations: dien, diencephalon; epi, ectodermal epithelium of the face; FNP, fronto-nasal primordium; MNP, mandibular primordium; neu, neuroepithelium of the brain; $\mathrm{ncm}$, neural crest-derived mesenchyme; tel, telencephalon. 
aspects of the molecular basis of brain-skull interactions have been clarified in amniote model organisms chick (Marcucio et al., 2005) and mouse (Bachiller at al., 2000). However, the existence of such interactions is less well understood in nonamniote tetrapods. An increase in our understanding of developmental patterns in the amphibian lineage can then clarify the presence or absence of patterns common to all tetrapods and thus shed light on whether or not there is deep conservation of brainskull interactions across Tetrapoda.

The main hypothesis of this thesis posits that removal of the forebrain will disrupt the development of craniofacial elements. This may pertain to certain key structures such as palatal, rostral, maxillary, and mandibular development, as well as interfere with development of the olfactory and optic sensory systems.

In order to address this hypothesis, in Chapter 2, I establish the normal development of the skull of Xenopus laevis by augmenting the widely used Nieuwkoop and Faber (NF) (1994) staging series that is based primarily on external anatomy. I document the development of the skull in figures of each NF stage, and further describe skull development in terms of variation observed, differing from previous studies of skull development in X. laevis (e.g., Trueb and Hanken, 1992). These data create a baseline of normal development at each stage, documenting the appearance of cartilaginous and ossified structures and facilitating comparisons with morphologies obtained when normal development is perturbed, as is the case in Chapter 3.

In Chapter 3, I conduct forebrain ablation experiments in embryonic $X$. laevis. This was done by removing the forebrain tissue, as well as the overlying dorsal skin, with a tungsten needle. The aim was to investigate the physical changes to the skull 
that occur as a result of forebrain disruption. Previous brain resection experiments performed on $X$. laevis focused on the role of the forebrain in axial patterning (Herrera-Rincon, 2017). Other research on genetic aspects of forebrain development in chick revealed disruption to telencephalic development may result in craniofacial deformities (Abzhanov and Tabin, 2004; Marcucio et al., 2005). Thus it is predicted that removing the tissue emanating these morphogenetic signals may cause downstream effects on the development of the skull. By comparing the post-surgery tadpoles to the normal developmental series from Chapter 2, I identify several resulting changes in the skull, and several changes in the morphology and development of the nasal capsules and eyes. Following this, inferences into which signaling pathways may have been impacted are made by comparing the current results with that of previous research.

In Chapter 4, I summarize my findings and relate them to our current understanding of $X$. laevis skull development. I propose potential research avenues as extensions of this current project, as this thesis raises several important questions of cranial and neural evolutionary patterns. I conclude by noting this MSc thesis helps to further our understanding of the influence of the forebrain on cranial development, and reveals the potential that integrating an evolutionary and molecular approach has on elucidating fundamental aspects of skull evolution. The approach of investigating development in an extant amphibian species to better understand that of extinct species can help us gain new insights into "how" the brain has influenced the evolution of the vertebrate skull - information that is not entirely attainable based on fossils alone. 


\section{Chapter 2: Normal skull development in Xenopus laevis - a complement to the Nieuwkoop and Faber staging series}

Note: This chapter discusses data that is part of a manuscript which was a collaborative project between Jade Atkins and myself. Much of the work was done together as it pertains to both of our theses, therefore there are similarities in written text between.

\section{1: Introduction}

Amphibians are members of the earliest-diverging branch of living, terrestrial vertebrates, and as such have been used extensively in studies of evolution and development. Notably, several recent publications have utilised amphibian models, such as the frog Xenopus laevis, to address questions related to the development of cranial anatomy (Elliot et al., 2013; Maddin et al., 2016; Herrera-Rincon et al., 2017; Lukas and Olsson, 2018). Xenopus laevis has a long history as a model organism in the fields of genetics and development (Heasman et al, 2006; Harland and Grainger, 2011; Yang et al, 2015; Session et al, 2016; Lukas and Olsson, 2018). Its relative ease of care and maintenance, with easily accessible oocytes makes Xenopus an ideal candidate for early developmental studies (Dumont, 1972).

Staging tables are fundamental tools in many developmental studies. They provide a baseline of normal development and establish a means to make comparisons of developmental events both within and across studies. The current, most widely used developmental staging series for Xenopus is that of Nieuwkoop and Faber (1994). Whilst this staging table provides extensive detail on developing external anatomy, allowing us to stage embryos through tadpole stages until the end of metamorphosis, it provides few details on internal anatomy, leaving room for error in staging specimens when key external features are missing or damaged. Other researchers have attempted to augment the table of Nieuwkoop and Faber (1994) with 
additional features (e.g., cartilages, muscles), or have developed novel staging tables based on different aspects of the animal (e.g., ossification sequence of the skull) (Trueb and Hanken, 1992; Ziermann and Olsson, 2007; Zahn et al., 2017; Lukas and Olsson, 2018). While these efforts provide valuable information about Xenopus development, they either do not include photographs and illustrations of each stage of development, which is critical for staging purposes, or the developmental data provided cannot be easily integrated (i.e. using the same described stages) within the already well-established Nieuwkoop and Faber (1994) series.

Given the growing use of $X$. laevis in studies of vertebrate cranial evolution and development, the need for a complete and detailed staging series of the skull is increasingly apparent (Elliot et al., 2018; Herrera-Rincon et al., 2017; Piekarski et al., 2014). Any study that manipulates development of the skull requires a detailed baseline of normal development in order to compare experimental animals and interpret results. Additionally, both Trueb and Hanken (1992) and Lukas and Olsson (2018) previously recognized skull development in Xenopus is variable; however, this variation was not described in detail, making it difficult to properly stage specimens with conflicting stage features. Therefore, to remedy these problems, it is necessary to re-examine and document the chondrification and ossification patterns of the skull in X. laevis, noting variation, for each Nieuwkoop and Faber stage (1994).

In this chapter, my aim is to provide a thorough staging table that describes the normal development of the skull of Xenopus laevis, augmenting the widely used stages of Nieuwkoop and Faber (1994). The stages described here encompass the first stage when the final chondrocranial structure appears - stage 41 - through to the completion of external metamorphosis - stage 66. As it is the most widely used staging table, describing skull development within the Nieuwkoop and Faber (1994) 
stages will ensure our data will be maximally integratable with the greatest amount of past and future research on Xenopus development. I further describe temporal and morphological variation observed at each stage. Together these data will allow researchers to assign specimens to the Nieuwkoop and Faber (1994) stages when only skull anatomy is present, or when aspects of skull development appear to be in conflict with any one stage. I also describe the ossification sequence in my dataset and highlight areas that contrast with sequences obtained in previous studies. Together this dataset provides the needed baseline of normal development for future studies that incorporate experimental development.

\section{$\underline{2.2}$ Methods}

\subsection{1: Animal Care and Breeding}

Breeding colony of adult Xenopus laevis is maintained in the Maddin Lab at Carleton University, Ottawa, Canada (original source: Marine Biology Laboratories, Massachusetts). All Xenopus procedures used in this study are in accordance with the Canadian Council on Animal Care and Carleton University's Animal Care Committee approved animal use protocols (AUP \#102952, 103203). Spawning was induced by initially injecting a female with 50 IU of human chorionic gonadotropin (HCG; Sigma C1063). Forty-eight hours later, the female received a second HCG injection of 500 IU. Concurrently, a male was injected with 50 IU of HCG. Upon completion of injections, both frogs were placed into one nuptial tank with tubing and artificial foliage. Embryos were collected the next morning and dejellied using a $2 \%$ buffered solution of L-Cysteine free base (Fisher Scientific BP376100). Embryos were then left to develop in agar-lined Petri dishes filled with $10 \%$ Holtfreter's solution at room temperature until stage 46 . At this stage, they were transferred to tanks with $20 \%$ 
Holtfreter's solution. Tadpoles were fed Tropical Pro Defence Hi-Protein food once daily when they were able to feed.

\subsection{2: Staging and Fixation}

Xenopus tadpoles were observed daily and staged according to Nieuwkoop and Faber (1994). When larvae reached the desired stage, they were anesthetized by adding a few drops of a neutral-buffered 4\% MS-222 solution (Sigma-Aldrich E10521, St. Louis, MO) to their containers. Once vital signs ceased, specimens were transferred to $10 \%$ neutral-buffered formalin (NBF, Thermo Fisher Scientific SF1004, Waltham, MA) and left to fix overnight at room temperature. Following fixation, specimens were rinsed in tap water for $2 \mathrm{hrs}$ and dehydrated through a series of ethanol in water dilutions ( 1 hour in each: $30 \%, 50 \%$ ) to a final storage solution of $70 \%$ ethanol.

\subsection{3: Tissue Staining}

For visualization of cartilage, specimens were placed in a solution of Alcian blue for up to six hours, time depending on specimen size (8GX, Acros Organics A3157) (20 mL acetic acid, $75 \mathrm{~mL}$ of $95 \%$ ethanol, $0.1 \mathrm{~g}$ Alcian blue). Specimens were then rehydrated through an ethanol series ( 1 hour in each: $70 \%, 50 \%, 30 \%$, and distilled water). Following rehydration specimens were placed in a solution of trypsin and saturated borate $\left(30 \%\right.$ saturated borate, $1 \%$ trypsin) in a $37^{\circ} \mathrm{C}$ incubator until the specimen's tissue was soft and about $50 \%$ cleared. To finish clearing and prepare the specimen for long-term storage, specimens were passed through a $1 \%$ potassium hydroxide $(\mathrm{KOH})$ in glycerol series (approximately one hour in each: 3:1, 1:1, and 1:3 $1 \% \mathrm{KOH}$ to glycerol) and then into $100 \%$ glycerol for final storage. For Xenopus, 
internal and external anatomy do not always correlate (Lukas and Olsson, 2018) - the internal anatomy was studied using descriptions from Trueb and Hanken (1992) while noting the variation from the determined Nieuwkoop and Faber stages (1994).

For visualization of bone, specimens were placed in a $1 \%$ Alizarin red $\mathrm{S}$ (Sigma-Aldrich A5533) in 1\% potassium hydroxide $(\mathrm{KOH})$ solution for one hour. Specimens were then placed into a solution of trypsin and saturated borate $(30 \%$ saturated borate, $1 \%$ trypsin) in a $37^{\circ} \mathrm{C}$ incubator until the specimen's tissue was soft and about $50 \%$ cleared. To finish clearing and prepare the specimen for long-term storage, specimens were passed through a $1 \% \mathrm{KOH}$ in glycerol series (approximately one hour in each: $3: 1,1: 1$, and $1: 31 \% \mathrm{KOH}$ to glycerol) and then into $100 \%$ glycerol for final storage. Imaging for all cleared and stained specimens was conducted using Zeiss SteREO Discovery.V8 stereo microscope. Illustrations were completed using Inkscape.

\section{3: Results}

Description of specimens begins at NF stage 41. This is the first stage when all the cartilaginous components of the cranium are present (i.e., first appearance of the final structure to chondrify - the occipital arch) and represents the span of stages that will be investigated in Chapter 3 (NF stage 41 to 51). From NF stage 54 onward, ossification has begun. From this stage forward, each stage is described based on the state of the ossifications only. 


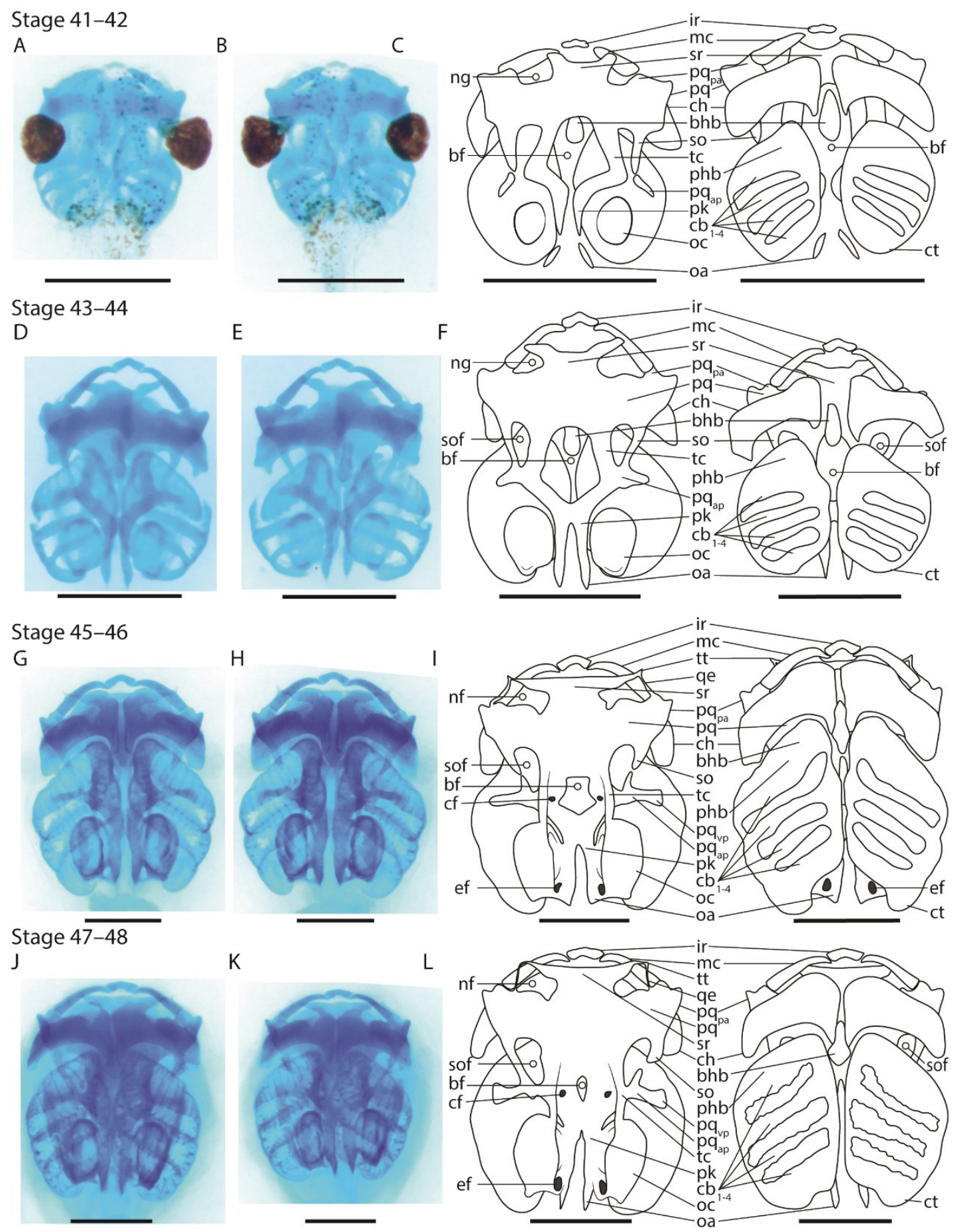

Figure 2.1. NF stages 41 to 48, cartilaginous skull development. From left to right, each row consists of a photograph in dorsal (left) and ventral (right) view, with an illustration in dorsal (left) and ventral (right) view. A-C, NF stage 41-42; D-F, NF stage 43-44; G-I, NF stage 45-46; J-L, NF stage 47-48. Scale bars equal $1 \mathrm{~mm}$. Abbreviations: bf, basicranial fenestra; bhb, basihyobranchial; cb, ceratobranchials; cf, carotid foramen; ch, ceratohyal; ct, commissura terminalis; ef, exoccipital foramen; ir, infrarostral; mc, Meckel's cartilage; nf, nasal foramen; ng, nasal groove; oa, occipital arches; oc, otic capsule; phb, planum hyobranchiale; pk, parachordal cartilage; $\mathrm{pq}_{\mathrm{ap}}$, ascending process of the palatoquadrate; $\mathrm{pq}$ a , pars articularis of the palatoquadrate; $\mathrm{pq}_{\mathrm{vp}}$, ventrolateral process of the palatoquadrate; qe, quadratoethmoidal; so, subocular cartilage; sof, suboccular fenestra; sr, suprarostral plate; tc, trabecular cartilage; tt, tenticular cartilage. 


\section{NF Stages 41 and $42(n=4)$}

Stage 41 and 42 specimens are very similar in degree of development of the chondrocranium and so are described here together. At these stages, the suprarostral plate is the dominant anterodorsal feature of the chondrocranium (Fig. 2.1A-C). In pipid species, the suprarostral plate is one large, unpaired structure that consists of the trabecular cartilages, the ethmoid plate (also called the anterior process of the ethmoid plate by some authors; e.g., De Sá and Swart, 1999) and the suprarostral cartilage (e.g., Trueb and Hanken, 1992; De Sá and Swart, 1999; Lukas and Olsson, 2018). Therefore, while other cartilages like the palatoquadrate, the subocular, and the parachordal cartilages initially form as discrete structures and later merge with the suprarostral plate, the trabecular, ethmoid, and suprarostral cartilages do not start their development as separate elements in X. laevis (Lukas and Olsson, 2018).

The anterior most margin of the suprarostral plate is incised deeply by what will later become the nasal foramina. Along the lateral margins of the anterior portion of the suprarostral plate is the larval pars articularis of the palatoquadrate, which articulates with Meckel's cartilage (Fig. 2.1A-C). Two pairs of processes extend posteriorly from the suprarostral plate (Fig. 2.1A-C). The lateral pair of processes are the subocular cartilages, and slightly posterior and lateral to the subocular cartilages is a separate pair of cartilaginous elements (Fig. 2.1A-C). These elements are the ascending processes of the palatoquadrate. The medial pair of posterior extensions are the trabecular cartilages, which are continuous posteriorly with the parachordal cartilages (Fig. 2.1A-C) and between them is a large fenestra that is open posteriorly. This is the basicranial fenestra. The trabecular plus parachordal cartilage processes extend to a level that is either anterior to or between the otic capsules (Fig. 2.1A-C). The otic capsule is not attached to other cartilaginous elements at this early stage of 
development (Fig. 2.1A-C). The otic capsule is more chondrified laterally than medially. The posteriormost element of the chondrocranium at these stages is the occipital arch. Here the occipital arch appears as a pair of ovoid bodies of cartilage posterior to the posterior tip of the parachordals (Fig. 2.1A-C).

The ventral view of the skull is dominated by the large hyobranchial apparatus; however, just anterior to the hyobranchial apparatus are the three elements of the lower jaw (Fig. 2.1A-C). The infrarostral cartilage has started chondrification and is faintly present at the midline (Fig. 2.1A-C). It is the anteriormost element. Meckel's cartilages occur lateral to the infrarostral cartilage. The Meckel's cartilages curve laterally and posteriorly, where they meet the palatoquadrates (Fig. 2.1A-C). With respect to the hyobranchial apparatus, the anteriormost elements are the paired ceratohyals (Fig. 2.1A-C). The ceratohyals are broad plates that curve posterolaterally. The medial portion of the ceratohyal also curves posteriorly and towards the ventral surface (Fig. 2.1A-C). Between the ceratohyals is the basihyobranchial. This teardrop shaped element sits posterior to the ceratohyals (Fig. 2.1A-C). The branchial basket occurs posterior to the ceratohyals and basihyobranchial and consists of the planum hypobranchiale and the four ceratobranchials. From anterior to posterior, within the branchial basket, is the planum hypobranchiale, which is a broad plate, and the first through fourth ceratobranchials (Fig. 2.1A-C). Along the distal and posterior edge of the branchial basket is the commissura terminalis, which connects all four ceratobranchial elements distally. Between each ceratobranchial element are the open pharyngeal clefts. 


\section{NF Stages 43 and $44(n=7)$}

Specimens at NF stages 43 and 44 are also described together here, as the degree of development and shape of the chondrocranium is largely indistinguishable. In the anterior and lateral portion of the suprarostral plates, the incisions for the nasal foramina have become partially closed anteriorly by extensions of the suprarostral plate, giving them the appearance of deepening (Fig. 2.1D-F). On the posterior margin of the suprarostral plate, the subocular cartilages have now merged with the ascending process of the palatoquadrate (Fig. 2.1D-F). This process of the palatoquadrate is also now longer and more robust. This is the first stage where the subocular fenestra may appear complete but is variable among the specimens examined here (e.g., the subocular fenestra is complete on both sides of the chondrocranium in two specimens, is complete on one side only in three specimens, and has not yet closed on either side in two specimens). When present, the subocular fenestra occurs between the subocular cartilage and the trabecular cartilage (Fig. 2.1D-F).

Posteriorly, the parachordals have met along the midline in two specimens, thereby completing the basicranial fenestra (Fig. 2.1D-F). Posterior to the basicranial fenestra, the parachordal cartilages remain separated from one another, on either side of the notochord (Fig. 2.1D-F). The parachordal cartilages have now merged with the occipital arch in three of the seven specimens examined, creating a long process that extends to just posterior to the otic capsules (Fig. 2.1D-F). The otic capsules are more robust at NF stages 43 and 44 than the previous stages, and now have grown closer to the lateral edges of the parachordal cartilages (Fig. 2.1D-F).

In the lower jaw, the infrarostral cartilage has made contact with Meckel's cartilage on each side. Meckel's cartilage is also now more elongate and curved 
posterolaterally (Fig. 2.1D-F). On the ventral surface, the ceratohyals have developed a more prominent posteriorly directed point at their lateral limits, but otherwise the hyobranchial apparatus appears relatively similar to that of the previous stage (Fig. 2.1D-F).

\section{NF Stages 45 and $46(n=10)$}

By NF stages 45 and 46, all of the various cartilaginous elements on the dorsal surface are now connected to one another in every specimen, including the otic capsules, which were previously separated from the other cartilaginous elements (Fig. 2.1). In the anterior region of the dorsal surface, the nasal foramina are complete in all specimens by NF stage 45 . The foramina are bordered anteriorly, medially and posteriorly by the suprarostral plate and laterally by the quadratoethmoidal, an extension of the palatoquadrate pars articularis (Fig. 2.1G-I). The quadratoethmoidal and the anterior projection of the suprarostral plate meet anteriorly to form the base of the tentacular cartilage. In some specimens at these stages, the tentacular cartilage already has an anterior projection (e.g., Fig. 2.1I). The ascending process of the palatoquadrate has once again changed shape, it has grown longer along its proximal to distal axis. In two specimens, the ventrolateral process of the palatoquadrate is present (Fig. 2.1I). This process is attached to the distal end of the ascending process of the palatoquadrate.

Along the midline of the skull in five out of ten specimens, the frontoparietal fontanelle is now defined. This fontanelle represents the space that will accommodate the future frontoparietal on the skull roof (Fig. 2.1I, midline). It is bordered anteriorly by the suprarostral plate, and on either side by the trabecular cartilages. The margins of the basicranial fenestra are now closed in all specimens examined, but the size of 
the fenestra varies among specimens. In three specimens there are paired carotid foramina on either side of the basicranial fenestra that accommodate the carotid arteries (Fig. 2.1G-I; Lukas and Olsson, 2017). Posterior to the basicranial fenestra, the parachordal cartilages remain separated by the notochord. In every specimen, the parachordal and occipital arch have met and in one specimen the metotic fissure - the gap between the occipital arch and otic capsule - has closed to form the metotic foramen (Fig. 2.1I; Clack, 2002). This is the foramen that transmits cranial nerve $\mathrm{X}$ (Naumann and Olsson, 2017; Quinzio and Fabrezi, 2018). The otic capsules are now attached to the rest of the chondrocranium via the trabecular cartilage dorsally and the parachordal cartilage ventrally (Fig $1 \mathrm{G}-\mathrm{I})$.

The elements of the lower jaw are more robust, but not significantly different in shape from the previous stages. The ceratohyals have become more robust and more closely approach one another at the midline. The basihyobranchial now sits in a groove between the two ceratohyals. In five of the ten specimens examined, the branchial basket has elongated along the anterior to posterior axis, and now is more oval in shape instead of being more circular (Fig. 2.1G-I).

\section{NF Stages 47 and $48(n=10)$}

At NF stage 47 and 48, the anterior portion of the suprarostral plate is overall similar in morphology to that in the previous stages (Fig. 2.1J-L). By now, the ventrolateral processes of the palatoquadrates are consistently present in all specimens (Fig. 2.1J-L). The basicranial fenestra continues to be variable in size, but in most specimens, it has shrunk in size to around $250 \mu \mathrm{m}$ long and $100 \mu \mathrm{m}$ wide, situated between the trabecular cartilages and visible dorsally through the frontoparietal fontanelle (Fig. 2.1J-L). The metotic foramen is consistently present in all specimens 
now (Fig. 2.1J-L). Additionally, there are small cartilaginous processes coming off the posterolateral corners of otic capsules. These are the muscular processes of the otic capsules.

The structures of the lower jaw and hyobranchial apparatus are similar in degree of development to those of the previous stages (Fig. 2.1J-L).

\section{NF Stage $49(n=8)$}

At NF stage 49, the tentacular cartilage is now consistently present as an anterolaterally projecting point at the apex of the quadratoethmoidal cartilage and the anterior projection of the suprarostral plate (Fig. 2.2A-C). In the posterior region of the chondrocranium, the occipital arch does not appear to protrude beyond the posterior margin of the otic capsule due to growth of the latter (Fig. 2.2A-C).

The only other morphological change that differentiates stage 49 from other stages is that in some specimens the basicranial fenestra has been completely infilled by cartilage. It is no longer present in three of the eight specimens examined (data not shown).

\section{NF Stages 50 and $51(n=7)$}

In the anterior region of the dorsal chondrocranium, the tentacular cartilage has grown into the tentacle structures, and they are now consistently present but remain short (1 $\mathrm{mm}$ or shorter in length; Fig. 2.2D-F). In the middle of the chondrocranium, the basicranial fenestra has been completely infilled by cartilage in all specimens examined (Fig. 2.2D-F). The ventrolateral process of the palatoquadrate is much larger and more prominent than in previous stages, projecting both anteriorly and posteriorly at its distal end (Fig. 2.2D-F). In the otic capsules, the epiotic eminences are visible for the first time, as well as the larval crista parotica. 

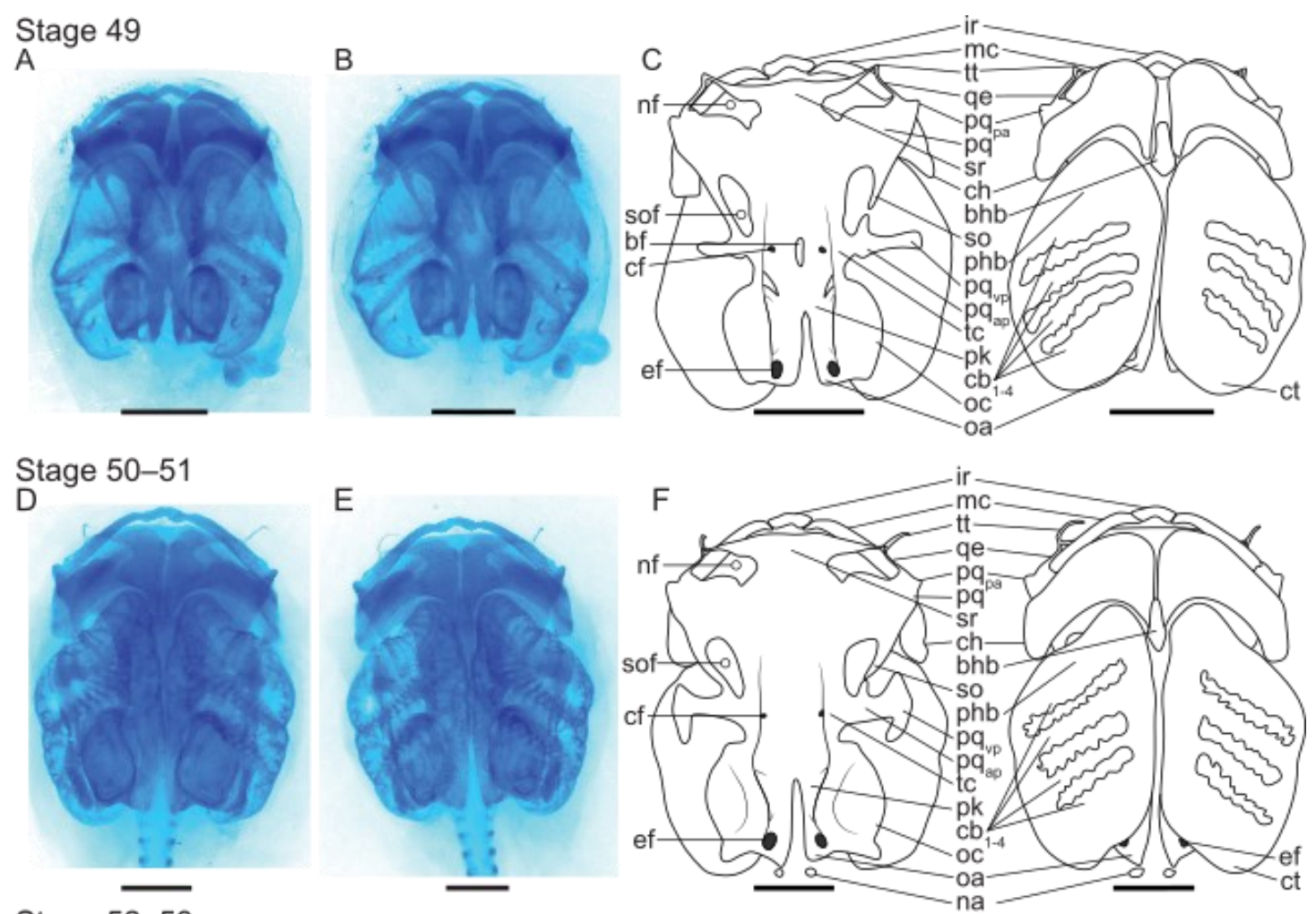

Stage 52-53

G

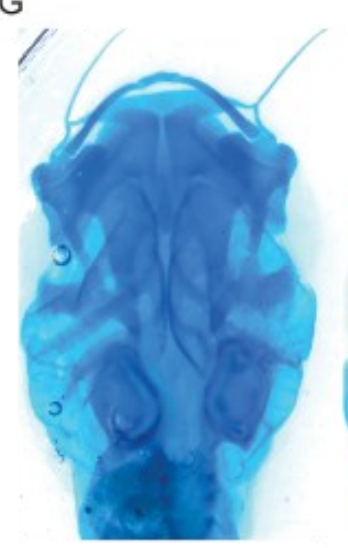

$\mathrm{H}$

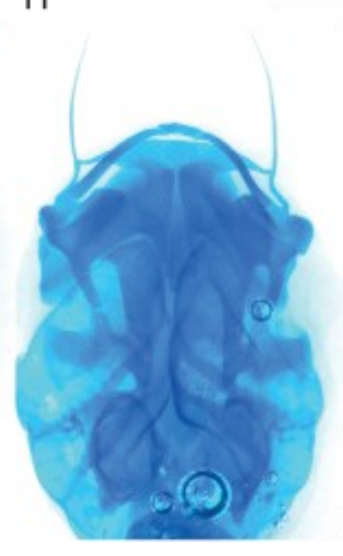

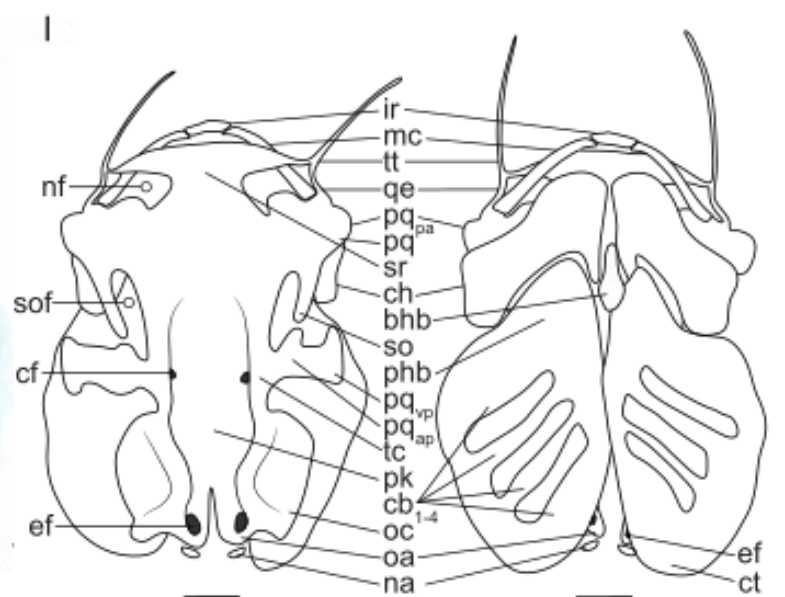

Figure $\overline{2.2}$. NF stages 49 to $\overline{53}$, cartilaginous skull development. From $\overline{l e f t}$ to right, each row consists of a photograph in dorsal (left) and ventral (right) view, with an illustration in dorsal (left) and ventral (right) view. A-C, NF stage 49; D-F, NF stage 50-51; G-NF stage 52-53. Scale bars equal $1 \mathrm{~mm}$. Abbreviations: na, neural arch; bf, basicranial fenestra; bhb, basihyobranchial; cb, ceratobranchials; cf, carotid foramen; ch, ceratohyal; ct, commisura terminalis; ef, exoccipital foramen; ir, infrarostral; mc, Meckel's cartilage; nf, nasal foramen; oa, occipital arches; oc, otic capsule; phb, planum hyobranchiale; $\mathrm{pk}$, parachordal cartilage; $\mathrm{pq}_{\mathrm{pa}}$, pars articularis of the palatoquadrate; $\mathrm{pq}_{\mathrm{ap}}$, ascending process of the palatoquadrate; $\mathrm{pq}_{\mathrm{v}}$, ventrolateral process of the palatoquadrate; qe, quadratoethmoidal; so, subocular cartilage; sof, suboccular fenestra; sr, suprarostral plate; tc, trabecular cartilage; tt, tenticular cartilage. 
The larval crista parotica is faint and does not appear in images as it is obscured by the otic capsules; however, when manually manipulating specimens, it can be seen located lateral and ventral to the otic capsules.

\section{NF Stages 52 and $53(n=9)$}

NF stages 52 and 53 represent the condition of the mature larval cartilage, as these stages are the final stages where only the chondrocranium is present. In general, specimens at NF stages 52 and 53 are more elongated anteroposteriorly than those of previous stages, which had consistently more rounder heads (Fig. 2.2G-I). The tentacular cartilage is now a long structure, consistently longer than $1 \mathrm{~mm}$, which was the maximum length observed in NF stages 50 and 51 (Fig. 2.2G-I). The frontoparietal fontenelle, which was present in previous stages, now resembles the shape and size of the future frontoparietal bone. The ascending process of the palatoquadrate has thickened along the anteroposterior axis (Fig. 2.2G-I). The protruding lower jaw is more strongly arched than that of previous stages, and the hyobranchial apparatus has expanded posteriorly, contributing to the more elongate shape of the head overall (Fig. 2.2G-I).

\section{NF Stages 54 and $55(n=19)$}

At NF stages 54 and 55, the first ossifications appear in twelve of the nineteen specimens examined. For the rest of the stages, only ossified elements will be described as they provide a better basis for staging than the more subtle changes taking place in the cartilages. In five specimens, only the parasphenoid is present, which makes it the first element to ossify in the skull (specimen not shown). In seven specimens, both the parasphenoid and the frontoparietal elements are present (Fig. 2.3A-C). The frontoparietal has started to ossify on the dorsal surface, with one 
A

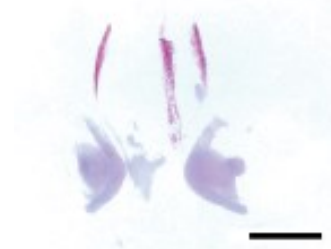

B

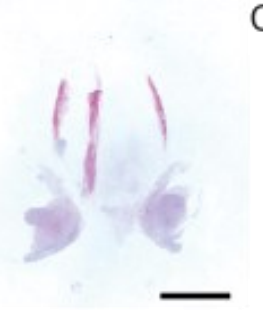

C

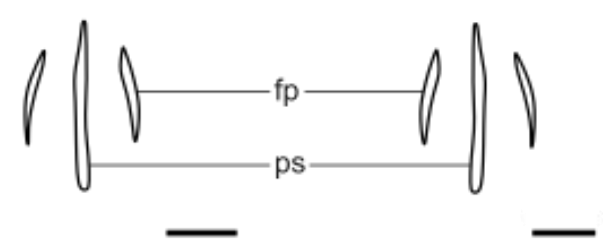

Stage 56

D

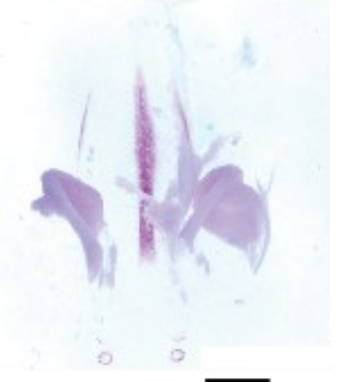

E

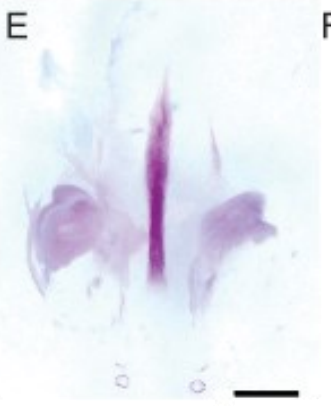

$\mathrm{F}$

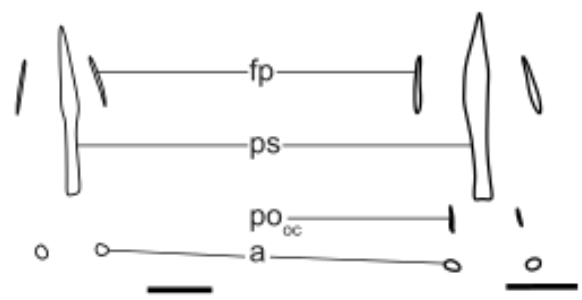

Stage 57
G

Stage 57
G

Stage 58-59
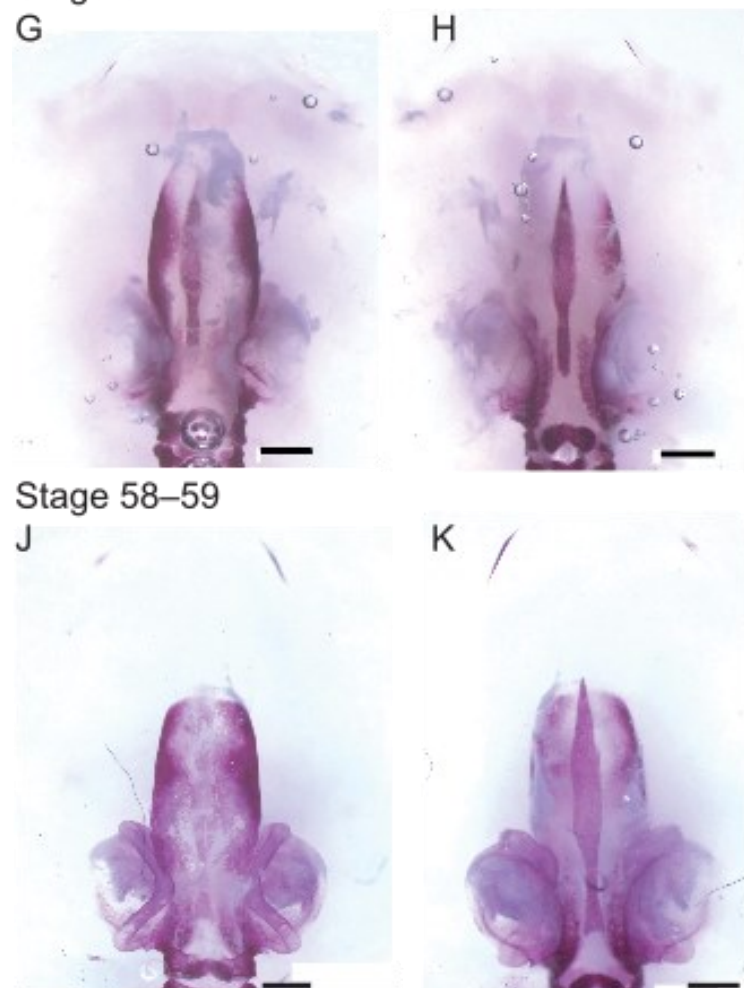

$\mathrm{K}$
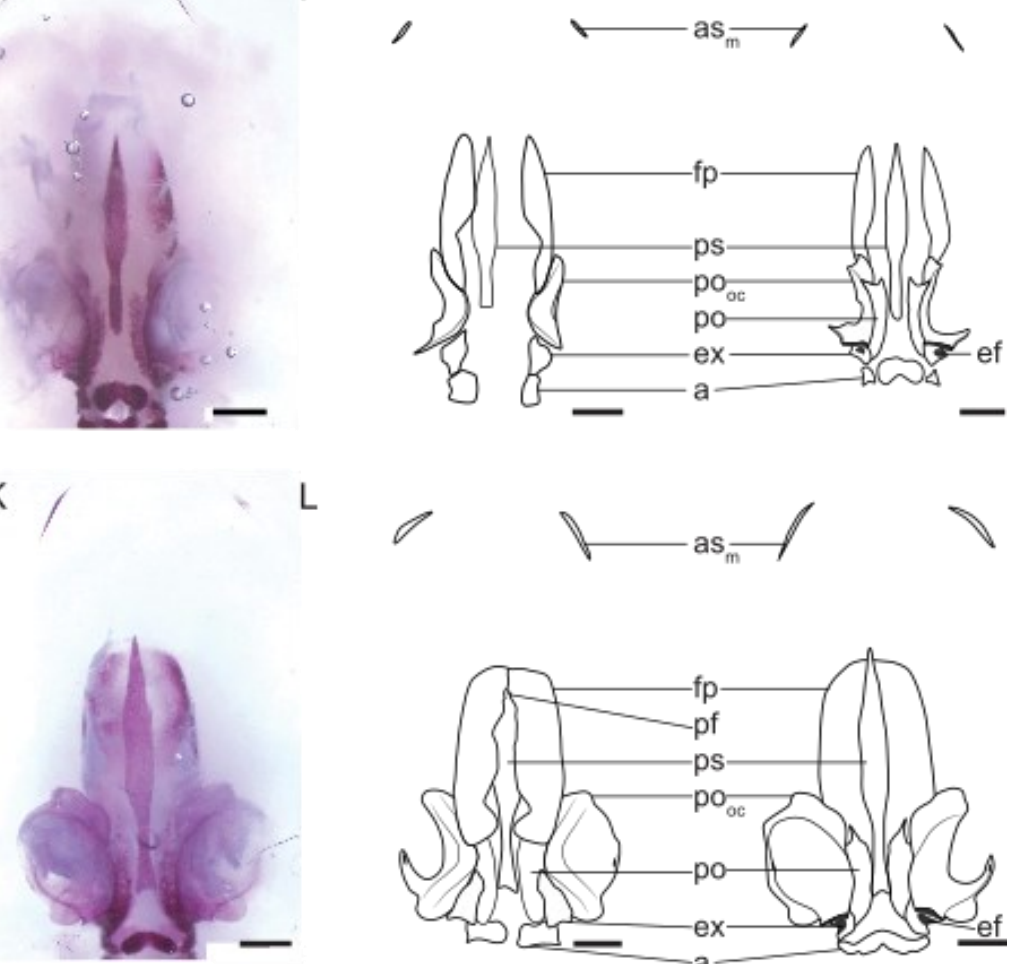

Figure 2.3. NF stages 54 to 59, osseous skull development. From left to right, each row consists of a photograph in dorsal (left) and ventral (right) view, with an illustration in dorsal (left) and ventral (right) view. A-C, NF stage 54-55; D-F, NF stage 56; G-I, NF stage 57; J-L, NF stage 58-59. Scale bars equal $1 \mathrm{~mm}$. Abbreviations: a, atlas; $\mathrm{as}_{\mathrm{m}}$, medial angulosplenial; ef, exoccipital foramen; ex, exoccipital; fp, frontoparietal; oc, otic capsule; po, prootic; ps, parasphenoid. 
ossification centre occurring on each side representing its lateral extent (Fig. 2.3A-C). Anteriorly, the frontoparietal terminates at approximately the same level as the parasphenoid and posteriorly it terminates anterior to the otic capsules (Fig. 2.3A-C).

On the palatal surface of the skull, the parasphenoid is present as a thin sliver of bone along the midline. Anteriorly, the parasphenoid terminates at a level equal to the midpoint of the eyes and posteriorly the parasphenoid terminates between the otic capsules (Fig. 2.3A-C).

\section{NF Stage $56(n=9)$}

As in NF stages 54 and 55, the elements that are present in the skull are the frontoparietal and the parasphenoid. In one specimen, only the parasphenoid is present, whereas in all other specimens examined both the frontoparietal and parasphenoid are present. For most specimens observed, the frontoparietal elements appear the same as in previous stages. However, in two specimens, the frontoparietals have started to ossify towards the midline of the skull. On the palatal surface of the skull, in all specimens observed, the parasphenoid has started to widen and lengthen (Fig. 2.3D-F). The parasphenoid now terminates posteriorly between the otic capsules. In one specimen, the prootic portion of the otic capsule has started to ossify ventromedially. In another specimen, ossification of the medial angulosplenial has just begun (this specimen not figured).

\section{NF Stage $57(n=8)$}

Specimens at NF stage 57 are variable in terms of the extent of ossification of various elements; however, they are less variable in terms of which elements have begun to ossify. All NF stage 57 specimens have a parasphenoid, frontoparietals, prootic ossifications in the otic capsules, exoccipitals, and the medial angulosplenials 
in the lower jaw (Fig. 2.3G-I). The frontoparietal fontanelle is visible at this stage as a grey outline along the midline of the skull (Fig. 2.3G, H). The ossifications of the frontoparietal within the frontoparietal fontanelle indicate that the frontoparietal has not yet fully ossified along its lateral extent (Fig. 2.3G-I). However, in most specimens the two elements have started to ossify medially towards its mate (Fig. 2.3G-I). On the palatal surface, the parasphenoid now better resembles the stereotypical spear shape typical of X. laevis with a pointed anterior end (Fig. 2.3G-I). The middle portion is now noticeably wider than the anterior and posterior portions (Fig. 2.3G-I).

In the posterior region of the skull, the level of ossification is variable among specimens at this stage. The otic capsules have begun to ossify in the medial region, forming a bony surface over the medial portion of the anterior and posterior semicircular canals dorsally and the medial portion of the floor of the otic capsule (Fig. 2.3G-I). This ossification corresponds to what is termed the prootic by Trueb and Hanken (1992). There is no evidence of a discrete opisthotic ossification. A large foramen is present on the posterior surface of the otic capsule. This foramen is figured but not named by Trueb and Hanken (1992). The exoccipitals are visible dorsally and ventrally, just posterior to the otic capsules. The exoccipital foramen is visible on the ventral surface of the exoccipital. This foramen transmits the vagus nerve, cranial nerve X (Naumann and Olsson, 2017; Quinzio and Fabrezi, 2018).

In the lower jaw, one element, the medial angulosplenial is present. It is a thin line of ossification along the medial surface of Meckel's cartilage. 
The frontoparietals have almost ossified to their full anterior and posterior extents along the lateral edges of the element, and continue to be variably ossified in their medial portion (Fig. 2.3J-L). By NF stage 58, the anterior portion of the frontoparietal appears as a square with rounded edges (Fig. 2.3J-L). The frontoparietals of some specimens have met at the midline anteriorly, and have begun to define the margins of the pineal foramen (specimens not figured). In one NF stage 59 specimen, the maxilla has just begun ossification in the anterior region of the skull (not visible in figured specimen).

In the posterior region of the skull, the otic capsules are now the dominant ossified elements due to expansion of the prootic ossification (Fig. 2.3J-L). Some variation exists in the extent of ossification of the otic capsules. Most specimens have at least the anterior and posterior semicircular canals covered in bone, and a smaller number of specimens now have ossification covering the horizontal semicircular canal (Fig. 2.3J-L). Outside of the otic capsule portion of the prootic ossification, the prootic has also changed shape medially to the otic capsules and has begun to ossify towards the midline of the skull and towards the anterior portion of the skull (Fig. 2.3J-L). Additionally, the prootic has ossified posteriorly and has started to articulate with the exoccipitals (Fig. 2.3J-L). When compared to previous stages, the exoccipital has become a wider element (Fig. 2.3).

In the lower jaw, the medial angulosplenial is longer than it was in NF stage 57 (Fig. 2.3J-L). 


\section{NF stage $60(n=2)$}

In one specimen at NF stage 60 , both paired maxillae and premaxillae are present, whereas in a second specimen both premaxillae are present but only the left maxilla is present. When present, the maxilla and premaxilla are the anteriormost elements (Fig. $2.4 \mathrm{~A}-\mathrm{C})$. A row of premaxillary and maxillary teeth is inconsistently present among specimens. When present, they are not yet mineralized at their bases and so appear as floating crowns (Fig. 2.4B). The nasals are variably present at this stage, and when present they are faint, crescent-shaped ossifications that occur just anterior to the frontoparietals (Fig. 2.4A-C). The frontoparietals have now reached their anterior and posterior limit on the lateral edges of the element, but are still variably ossified in the medial portion of these elements (Fig. 2.4A-C). In one specimen, they have nearly met along the midline in the central region of the element (specimen not figured), whereas in the second specimen the frontoparietals have met along the midline in the anterior region of the element (Fig. 2.4A-C). Both specimens have now completed ossification around the pineal foramen (Fig. 2.4B, C). The posterior portion of the lateral frontoparietals overlie the medial otic capsules as well as the anterior portion of the prootic (Fig. 2.4A).

The otic capsule remains variable in its extent of ossification, with one specimen showing complete ossification (specimen not figured), whereas in the other specimen only the area covering the semicircular canals is ossified (Fig. 2.4A-C). The medial portion of the otic capsules is more robustly ossified than observed in previous stages but otherwise has the same morphology as in NF stages 58 and 59, while the exoccipital continues to ossify posteriorly (Fig. 2.4A-C).

In the lower jaw, the medial angulosplenial is now more extensively ossified posteriorly, reaching the midpoint level of the eye (Fig. 2.4A-C). 

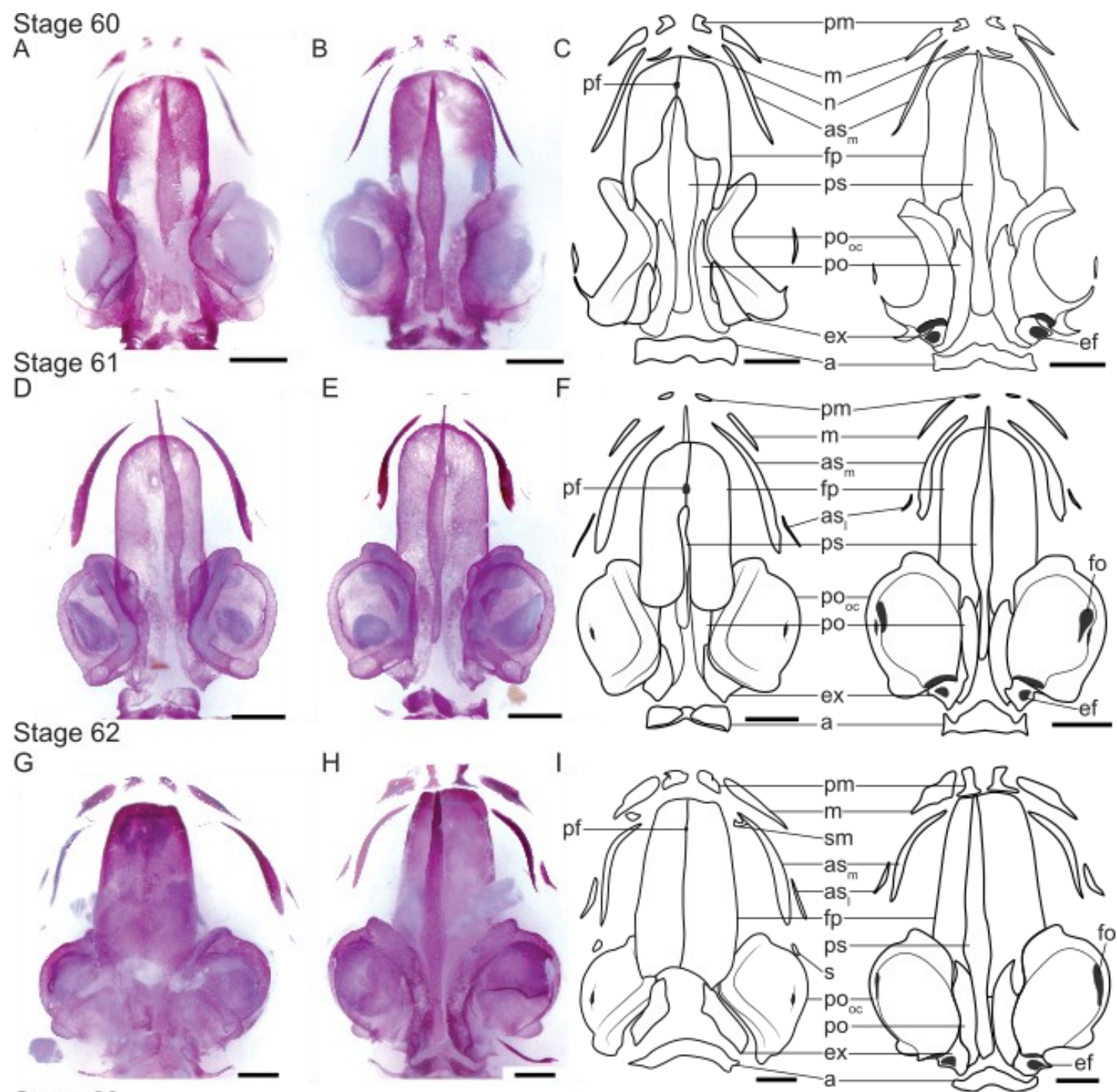

Stage 63
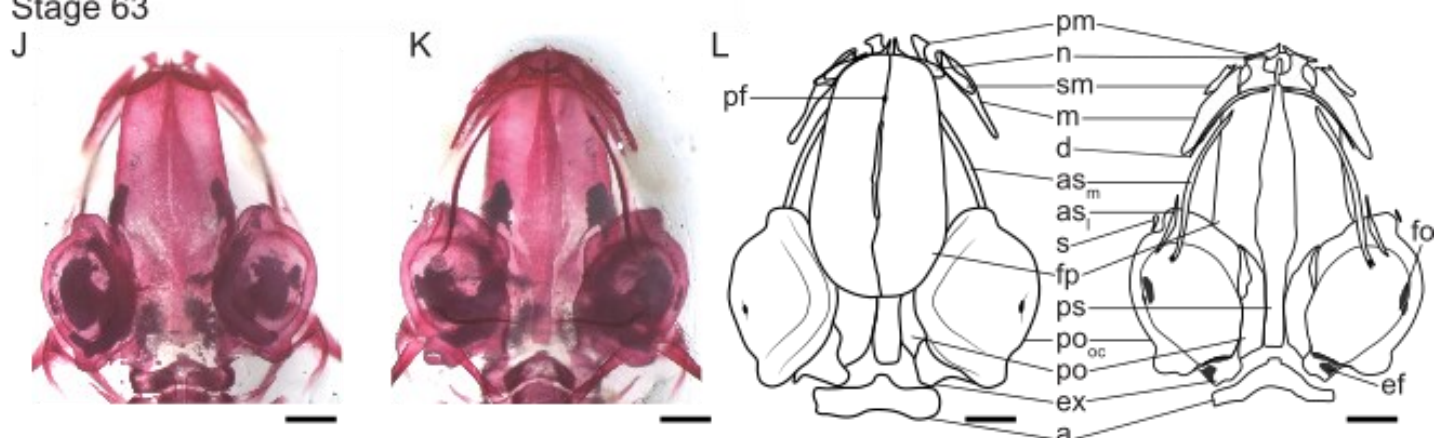

Figure 2.4. NF stages 60 to 63, osseous skull development. From left to right, each row consists of a photograph in dorsal (left) and ventral (right) view, with an illustration in dorsal (left) and ventral (right) view. A-C, NF stage 60; D-F, NF stage 61, upper and lower jaw elements were digitally rearticulated with the skull in panels $\mathrm{D}$ and $\mathrm{E}$, the composite photos were used as the basis of the line drawings in F; G-I, NF stage 62, upper and lower jaw elements were digitally rearticulated with the skull in panels $\mathrm{G}$ and $\mathrm{H}$, the composite photos were used as the basis of the line drawings in I; J-L, NF stage 63. Scale bars equal $1 \mathrm{~mm}$. Abbreviations: a, atlas; as m, medial angulosplenial; ası, lateral angulosplenial; d, dentary; ef, exoccipital foramen; ex, exoccipital; fo, foramen ovalis; fp, frontoparietal; $\mathrm{m}$, maxilla; $\mathrm{n}$, nasal; oc, otic capsule; pf, pineal foramen; pm, premaxilla; po, prootic; ps, parasphenoid; s, squamosal; sm, septomaxilla. 
NF stage $61(n=2)$

The maxilla, premaxilla, and nasal are still variably present elements by NF stage 61 . The nasal is present in one stage 61 specimen (specimen not figured) but not yet present in a second specimen (Fig. 2.4D-F). The row of teeth observed in one NF stage 60 specimen is not present in either of the NF stage 61 specimens, indicating variability in their presence across these two stages. The frontoparietals continue to be variably ossified in the medial portion of these elements, identical to morphologies seen in NF stage 60 (Fig. 2.4A-F). The otic capsule, however, is now consistently fully ossified (Fig. 2.4D-F). Each otic capsule has a large foramen visible on the ventrolateral surface; this is the fenestra ovalis (Fig. 2.4F). The anterior, posterior, and horizontal semicircular canals are now all covered in bone (Fig. 2.4D-F). The midline portion of the prootic has the same morphology on the dorsal and ventral surfaces as was observed in NF stages 58 through 60.

In the lower jaw the medial angulosplenial continues to ossify along the medial surface of Meckel's cartilage. The medial angulosplenial is more robust than that observed in previous stages (Fig. 2.4D-F). A second ossification, the lateral angulosplenial, is now variably present (Fig. 2.4D-F). The lateral angulosplenial, when present, is a very thin line of bone on the lateral surface of the lower jaw in the posterior region (Fig. 2.4D-F).

NF stage $62(n=4)$

By NF stage 62 , both the premaxilla and maxilla are consistently present. The premaxilla are now roughly $\mathrm{T}$-shaped, with the development of the dorsal alary process. The maxilla have ossified medially to the extent that they either nearly articulate (Fig. 2.4G-I) or do articulate with the lateral processes of the premaxillae. 
The tooth row first visible in stage 60 is now consistently present in all specimens, but continues to appear as a row of floating crowns (Fig. 2.4G-I). The nasals are still variably present. Septomaxillae are now also variably present. They appear on both sides in one specimen, on only the left side of a second specimen, and are not present in a third specimen. When present, the septomaxillae are faint lines of ossification that occur just lateral to the anterior portion of the frontoparietal (Fig. 2.4G-I). The frontoparietals now meet very closely along the midline (Fig. 2.4G-I). They may form a suture in the anterior and posterior regions of the bone; however, the middle portion of the suture is variably present, and a small gap may persist (Fig. 2.4G-I). The pineal foramen is consistently defined in all specimens at this stage (Fig. 2.4G-I). Anterior and lateral to the otic capsules are faint ossifications. This is the first appearance of the paired squamosals (Fig. 2.4G-I). The squamosals are present as thin lines of ossification anterolateral to the otic capsules. The medial margins of the prootic have also started to ossify towards the midline on both the dorsal and ventral surfaces (Fig. 2.4G-I).

In the lower jaw, the medial angulosplenial resembles the morphology of NF stage 61 , whereas the lateral angulosplenial is now consistently present but variable in extent of ossification. The lateral angulosplenial is a thin line of ossification on the posterior portion of the lower jaw.

\section{NF stage $63(n=3)$}

The lateral processes of the premaxilla have extended laterally and are wider than the alary process is tall (Fig. 2.4J-L). The premaxilla and maxilla have now ossified to meet and surround the bases of the teeth observed in previous stages (Fig.

2.4J-L). The septomaxilla is now present in all specimens on both sides of the skull, 
where it can be seen to be a rod-shaped bone. At this stage, the medial and lateral extents of the septomaxilla are well ossified, but ossification in the central portion is variable among specimens (Fig. 2.4J-L). In one specimen, there is little ossification in the central portion, whereas in a second specimen the central portion is completely ossified. The nasals are also consistently present and now the posterior portion of the nasals underlies the anterior frontoparietals (Fig. 2.4J-L).

On the palatal surface, the anterior portion of the parasphenoid comes to a point at a level that is variable between specimens. In one specimen, the anterior limit almost reaches the level of the alary process of the premaxilla (Fig. 2.4J-L), whereas in a second specimen the anterior limit is posterior to the premaxilla. One specimen shows signs of early ossification of the sphenethmoids. These ossifications appear as very thin lines running along the lateral edge of the widest point of the parasphenoid (not visible in the figured specimen). At NF stage 63, the prootic and exoccipital have changed shape on their dorsal surface. The medial portion of the otic capsule portion is much more extensively ossified than observed in NF stage 62 (Fig. 2.4J-L) and the posterior end of the exoccipital now has medially directed flange (Fig. 2.4J-L).

In the lower jaw, all three elements (dentary, medial and lateral angulosplenial) are now present and variably ossified (Fig. 2.4J-L). Anteriorly, the two halves of the dentary approach one another closely at the midline (Fig. 2.4J-L). Posteriorly, ossification of the dentary reaches the level of the posterior extent of the maxilla (Fig. 2.4J-L). The largest element, the medial angulosplenial, has an anterior extent that is either approximately level with the midpoint of the dentary or more posterior. Posteriorly, the medial angulosplenial is more extensively ossified and has almost ossified to the level of the posterior extent of the lower jaw (Fig. 2.4K-L). The 
lateral angulosplenial has grown in size along its anterior to posterior axis (Fig. 2.4JL).

NF stage $64(n=3)$

On the ventral surface of the maxilla and premaxilla elements, a second more lingually positioned row of teeth has started to form in the anterior portion of the maxilla in one specimen (Fig. 2.5A-C). This is the second series that occurs posterior and in between teeth present from the first series, as described by Shaw (1979). The labial series of teeth, the tooth row that was first to develop, are larger than the lingual series (Fig. 3.1). The suture between the frontoparietals may be partially obliterated by fusion of the two elements at this stage in the anterior region only (Fig. 2.5A-C). The suture is still visible posterior to the pineal foramen in all specimens observed at this stage (Fig. 2.5A-C).

On the palatal surface, ossification of the sphenethmoid is only present in one specimen and the morphology resembles that of NF stage 63. The pterygoid is now present on the palatal surface (Fig. 2.5A-C). This bone is triangular in shape with one point directed anteriorly, and the other two directed posterolaterally and posteromedially, respectively (Fig. $2.5 \mathrm{~A}-\mathrm{C}$ ). In the posterior region of the skull, anterior and lateral to the otic capsules, the squamosals have slightly changed in shape (Fig. 2.5A-C). The squamosal is now roughly $\mathrm{C}$-shaped, with the concavity opening posteriorly.

Elements of the lower jaw (dentary, medial and lateral angulosplenial) have increased in ossification. 
Stage 64

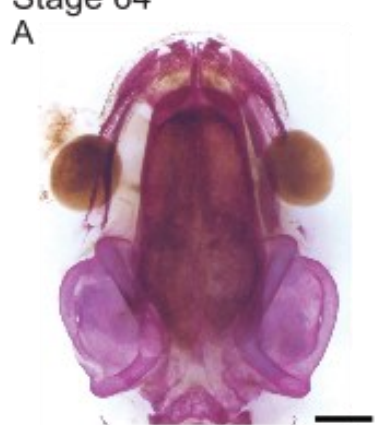

Stage 65

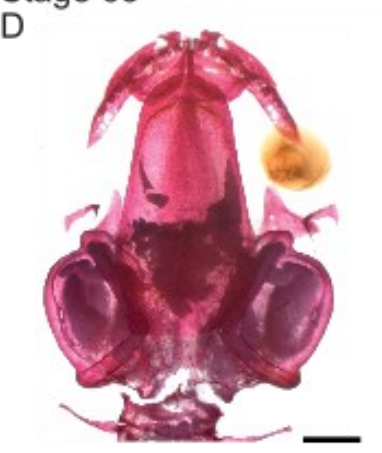

Stage 66

G

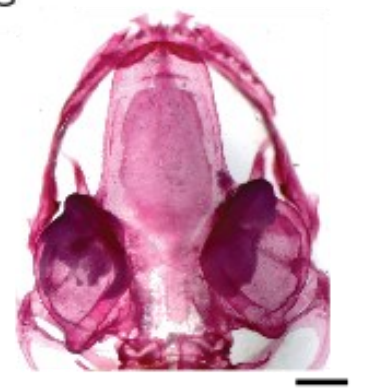

$B$
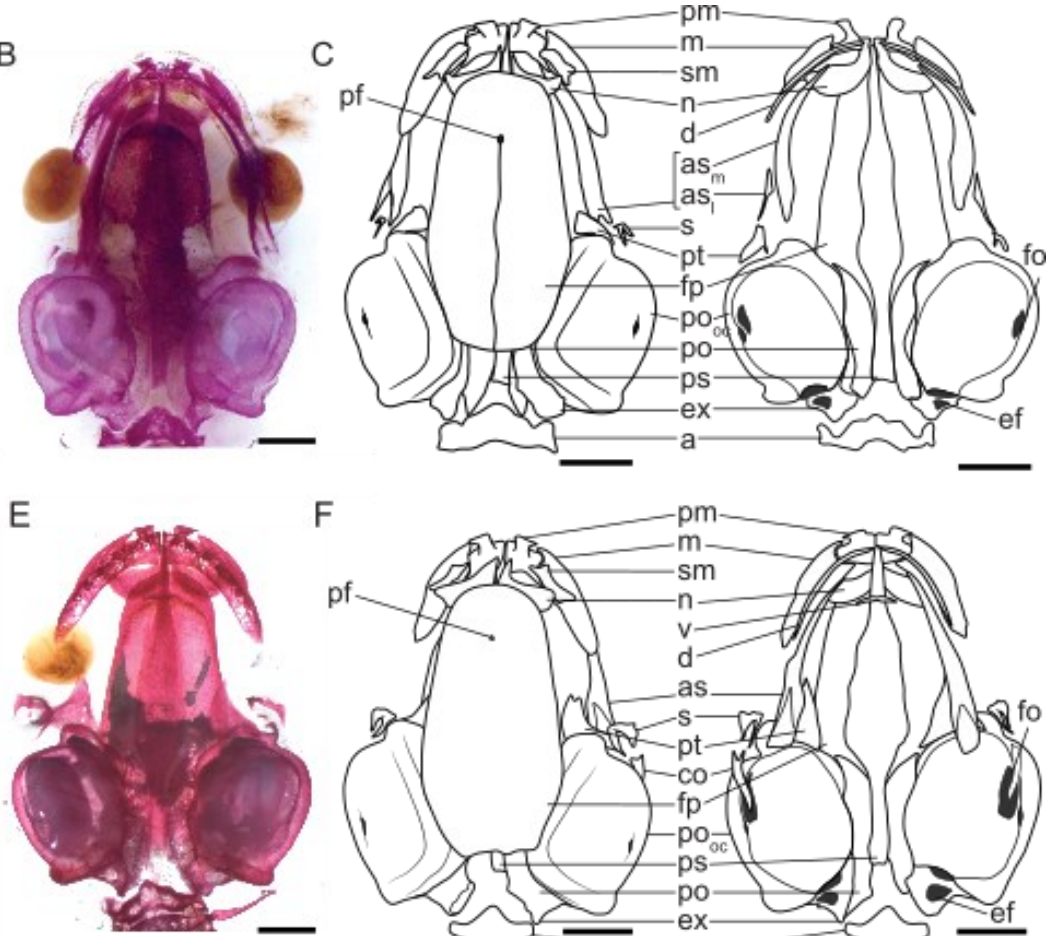

$\mathrm{F}$

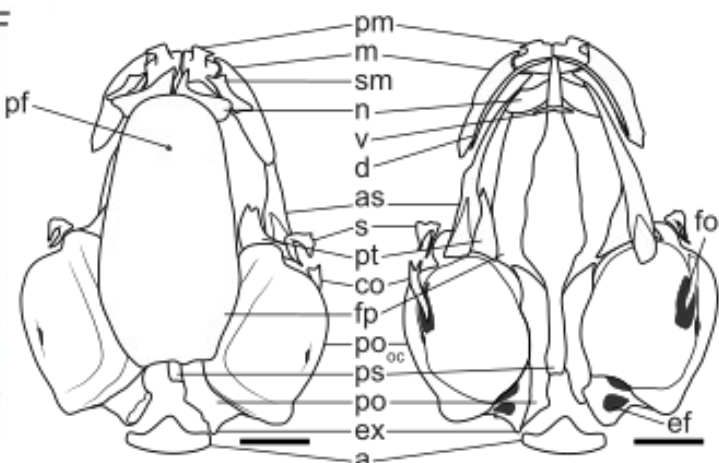

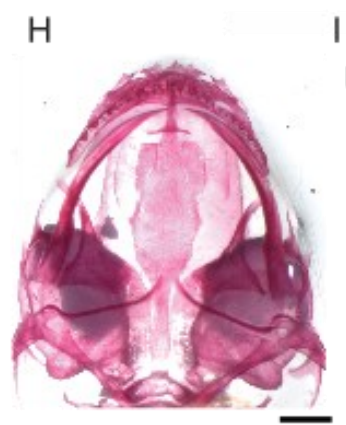

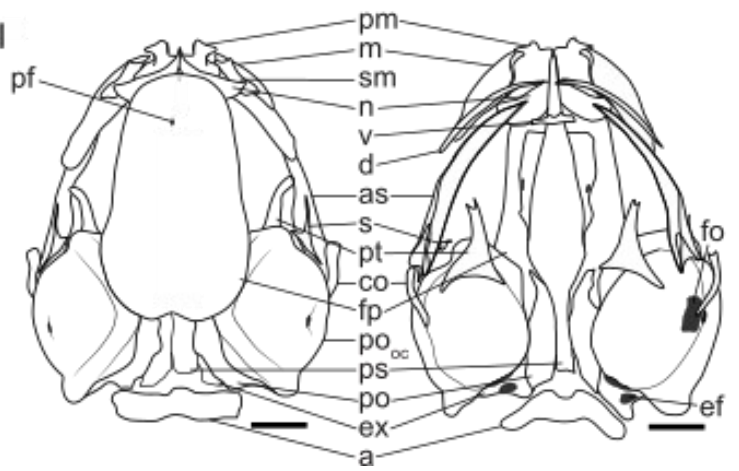

Figure 2.5. Stage 64 to 66 , osseous skull development. From left to right, each row consists of a photograph in dorsal (left) and ventral (right) view, with an illustration in dorsal (left) and ventral (right) view. A-C, stage 64; D-F, stage 65; G-I, stage 66. Scale bars equal $1 \mathrm{~mm}$. Abbreviations: a, atlas; as, angulosplenial with fused medial and lateral ossifications; $\mathrm{as}_{\mathrm{m}}$, medial angulosplenial; as, lateral angulosplenial; co, columella; d, dentary; ef, exoccipital foramen; ex, exoccipital; fo, foramen ovalis; fp, frontoparietal; $\mathrm{m}$, maxilla; $\mathrm{n}$, nasal; oc, otic capsule; pf, pineal foramen; pm, premaxilla; po, prootic; ps, parasphenoid; pt, pterygoid; s, squamosal; sm, septomaxilla; v, vomer. 
NF stage $65(n=4)$

On the ventral surface of the maxilla and premaxilla, two series of teeth are consistently present (Fig. 2.5D-F). The septomaxillae are broader and more robust in one specimen, where they now appear to be dumbbell shaped (Fig. 2.5D-F). The posterior portion of the septomaxilla now underlies the nasals (Fig. 2.5D-F). At the anterior and medial extent of the nasals, the two elements make contact with one another along their midline. The suture between the frontoparietals may be obliterated by this stage (as seen in one specimen), with a pineal foramen remaining located in the anterior half of the element, at a level approximately equal to the anterior extent of the eyes (Fig. 2.5D-F). In the other three specimens, the suture is only obliterated in the anterior portion (specimens not shown).

On the palatal surface, in the anterior region of the skull, the vomers are now present (Fig. 2.5D-F). The vomers appear as a small, singular element located ventral to the parasphenoid that arises from two ossification centres that subsequently fuse at the midline (Trueb and Hanken, 1992). The vomer is longer in its medial region, tapering to points at each lateral extent of the element (Fig. 2.5D-F). By NF stage 65, the anterior portion of the parasphenoid consistently almost reaches the level of between the alary processes of the premaxillae (Fig. 2.5D-F). This is the complete extent of the parasphenoid will grow anteriorly. On either side of the parasphenoid, the very thin lines of ossification that represent the sphenethmoid have thickened in one specimen (not shown) and are not yet visible in the others (Fig. 2.5D-F).

The dorsal end of the squamosal remains pointed, as in previous stages; however, the ventral end has widened to a blunt point (Fig. 2.5D-F). The columella (or stapes) is now present for the first time at NF stage 65 (specimen not shown). Its proximal end rests adjacent to the fenestra ovalis of the otic capsule, and distally 
extends anteriorly. Anterior and ventral to the otic capsules, the pterygoids are now larger, triangular elements than observed in previous stages, especially when viewed from the ventral surface (Fig. 2.5D-F)

In the lower jaw, the three elements (dentary, medial and lateral angulosplenial) have started to articulate with each other in some specimens. In one specimen, all three elements are articulated on the dorsal surface and in the posterior region of the lower jaw only on the left side (specimen not figured), while in a second specimen this morphology is present on both sides of the lower jaw (Fig. 2.5D-F). In two other specimens, only the medial and lateral angulosplenials have started to articulate on the dorsal surface and the dentary is still a separate element (specimens not shown).

\section{NF stage $66(n=4)$}

NF stage 66 is the final stage described by Nieuwkoop and Faber (1994). By this stage, the frog has virtually completed metamorphosis based on external features. The skull, however, has not completely finished development. As this staging series is meant to augment the pre-existing Nieuwkoop and Faber series (1994), which only covers until NF stage 66, this is our final described stage. The majority of postmetamorphic changes occur in the posterior region of the skull (Trueb and Hanken, 1992).

On the dorsal surface, the suture between the frontoparietals is entirely obliterated at this stage (Fig. 2.5G-I). On the palatal surface, on either side of the parasphenoid, the sphenethmoid has ossified laterally and is now visible in all specimens (Fig. 2.5G-I). These paired elements ossify lateral and dorsal to the parasphenoid, forming a cup-shape in cross-section, along with the parasphenoid 
below. The sphenethmoid is more extensively ossified in its anterior region and has a foramen located approximately midway along its anterior-posterior axis, this is for the optic nerve (Fig. 2.5G-I).

Morphological changes in the posterior portion of the skull are extensive. The dorsal portion of the squamosal now has an anteriorly directed process (Fig. 2.5H-I). The posterior apices of the triangular pterygoids have expanded such that the pterygoid now underlies the anterior portions of the otic capsules (Fig. 2.5H-I).

The elements of the lower jaw do not differ when compared to stage 65 , except that in all specimens the medial and lateral angulosplenial have now articulated in their posterior ends, as first observed in stage 65 (Fig. 2.5G-I).

\subsection{Discussion}

The present study represents the first descriptive staging table of normal skull development in the model species $X$. laevis that describes, with figures, the morphology and development of cranial cartilages, and the first appearances of bones from NF stage 41 to 66 according to Nieuwkoop and Faber (1994). While the work of previous researchers on $X$. laevis development described the sequence of ossification and the appearance of some bones, they did not include figures for every stage (Trueb and Hanken, 1992), did not account for temporal variation in ossification (Slater et al., 2009), or they focused on external head features (Nieuwkoop and Faber, 1994; Zahn et al., 2017). With this new, complementary staging table, researchers working on the skull can now confidently know at which stage or stages bones of interest are present, their morphology, and the temporal variation in the first appearance of each element at each stage of the already widely used Nieuwkoop and Faber (1994) staging table. 
We note several key differences between the present study and previous studies of skull development in $X$. laevis. These observations confirm the existence of temporal variation in the first appearances of ossification during X. laevis skull development, as has been noted by several researchers (Ziermann and Olsson, 2007; Lukas and Olsson, 2018; Trueb and Hanken, 1992). Summarized in Table A2, the following are particularly noteworthy in the degree of deviation between studies. In the present study, the parasphenoid was consistently the first element to ossify, whereas previous studies found that the frontoparietal was the first element to ossify (Table A2). The first appearance of the maxilla and premaxilla is found here to be both later and more variable than reported in previous studies (Table A2). For example, in the present study, the maxilla first appeared between NF stages 58 and 62, whereas the premaxilla first appeared between NF stages 60 and 62. In Trueb and Hanken (1992) both elements were consistently present by NF stage 59, whereas in Bernasconi (1951) they were present by NF stage 55. Additionally, the septomaxilla ossifies first at NF stage 62 in the current study, but at NF stage 60 in other studies (Table A2). Finally, in the present study, we note that the collumella is present only by NF stage 65, whereas in previous studies it was present by NF stage 60 (Table A2). Most of the other temporal variation in the first appearances of skull bones is variation by one or two stages. Some of these variations result in minor differences in the ossification sequence observed here with that of previous studies; however, for the most part, the ossification sequence is consistent across studies despite these temporal differences in first appearances of bones.

We found that the cartilaginous stages examined (i.e., NF stages 41 to 53) were less variable in their morphology than the stages where ossification was taking place. Among the most variable cartilaginous structures were the subocular fenestrae. 
The subocular fenestra had inconsistent timing of closure as a result of variability in the timing of the subocular bar fusion to the ascending process of the palatoquadrate. This fenestra was seen closed as early as NF stage 43 and as late as NF stage 53 . In addition to the variation in the timing of first appearances of bones, variation in the extent of ossification of an element, and thus its size, was variable within stages. This means that although a group of specimens had been assigned a given NF stage, they may not all display the same degree of ossification. The otic capsule showed much variability in ossification, even bilaterally within a single specimen (Fig. $2.3 \mathrm{~J}-\mathrm{L}$ ), as well as across stages. They always ossified in the medio-lateral direction but the extent of their lateral reach was inconsistent until full ossification at NF stage 61 . Finally, the frontoparietals ossified along their outer edges before continuing to grow medially. The extent of medial ossification of the frontoparietals is the source of variability between NF stage 60 to 64 . As such, the extent of ossification of the frontoparietal in an NF stage 60 specimen might resemble that of an NF stage 62 specimen and vice versa. Many other structures show slight inconsistencies between specimens of the same stage, such as the size of the basicranial fenestra, the degree of anteroposterior extension of the ventrolateral process of the palatoquadrate, and the time of appearance for the maxillary structures and the nasals; however, these variations only span one to two stages rather than the aforementioned structures which can span up to ten.

Reasons for the existence of temporal variation in the development of skull features, such as those observed here, have been proposed to be the result of environmental variations such as temperature, density, or the amount of light received by the tadpoles (Trueb and Hanken 1992). Notably, the stages where the most variation was observed (NF stages 55 to 62) correspond to both the initial spike in the 
concentration of thyroid hormones at the beginning of metamorphosis (NF stage 55) and to the peak of metamorphosis (NF stages 58 to 62) (Kawahara et al., 1991). It is thus possible that the reason the internal anatomy appears more variable relative to the external anatomy is the result of the rapid development of numerous components of the adult skull, in comparison to less complex changes in the external anatomy (e.g., tail resorption, limb extension) taking place during metamorphosis. We concluded that variation in ossification was not due to staining methods as our materials and methods were nearly identical to that of Trueb and Hanken (1992), which was our main baseline comparison.

For the purpose of this thesis, this staging table establishes a detailed baseline of normal skull development for $X$. laevis, both in terms of timing of developmental events (e.g., first appearance of skull structures) and normal morphology at each stage. The chondrocranium is considered especially important for the process of ossification by setting up a primary scaffolding to guide many dermal bones (Kawasaki and Richtsmeier, 2017; Pitirri et al., 2020). Although the chondrocranium and the dermatocranium evolved separately, with the former being the first to evolve (Cervantes-Diaz et al., 2017), the retention of the chondrocranium in all vertebrates has led to the assumption that it plays an essential role and is required for proper skull development (Hirasawa and Kuratani, 2015). The exact nature of the mechanisms underlying the relationship between the chondrocranium and the dermatocranium is not fully understood, but there is much information available regarding the interactions of specific cranial elements. McBratney-Owen et al. (2008) discuss in detail which chondrocranial elements in mouse act as templates for peri- and endochondral ossification of their respective cranial bones. Once their role as a 
template and dermal bone ossification is complete, several cartilages dissolve (Pitirri et al., 2020).

Chondrocranial-dermatocranial interactions are seen in several regions of the head - one example being Meckel's cartilage and the lower jaw bones (Kawasaki and Richtsmeier, 2017). Trueb and Hanken (1992) state that the angulosplenial, both the medial and lateral ossification centers, invest on the surface of Meckel's cartilage. The dentary, although initially a distinct structure, develops along the fusion point of Meckel's cartilage and the infrarostral. This is a key feature to note as one of the most affected structures from forebrain surgical manipulation is the lower jaw. Notably as a result of the forebrain removal, the posterior region of the skull (i.e., any structure posterior to the ascending process of the palatoquadrate) remained unaffected. Both of these features will be discussed in Chapter 3 .

While Meckel's cartilage is a critical structure in determining bone formation, it is not the only one. The frontoparietal fontanelle outlines the anterior brain cavity and, as its name would suggest, the initial shape the ossifying frontoparietal will take on. Also, the majority of the anterior skull elements require the trabecular cartilages and the suprarostral plate for normal development (Trueb and Hanken, 1992). The trabeculae and suprarostral plate are remodelled during the development of the nasal capsule which then, in suite, guide the ossification of several anterior elements such as the paired nasals and septomaxillae. As the trabecular and suprarostral plates dissolve, cartilaginous bridges remain between bone structures, and as such, interfering with their development may have large scale effects on the skull as a whole (Trueb and Hanken, 1992).

Knowing the role that the aforementioned cartilaginous structures play in guiding certain cranial bones will greatly help understand the implications of 
chondrocranial morphologies arising from surgical manipulation. In this thesis, the oldest of the surgical specimens reached around NF 49 to 51, which is not old enough to show first signs of ossification. Several hypotheses regarding the reason for the interruption of development are discussed in Chapter 3. Even though the specimens did not reach the stages of ossification, there is still valuable information to be gained from the stained chondrocrania of experimental specimens that can allow us to make inferences about the impacts malformed chondrocranial structures resulting from disruptions to brain development could have on the dermatocranium. These are discussed in Chapters 3 and 4. 


\section{Chapter 3: Surgical manipulation of Xenopus laevis embryonic forebrain to study its role in the structural development of the amphibian cranium}

\section{$\underline{\text { 3.1 Introduction }}$}

The coevolution of the brain and skull has been demonstrated in certain vertebrate lineages, namely mammals and birds. Several studies have utilized fatemapping, the fossil record, or the mechanical pressures of encephalization to demonstrate that skull expansion is correlated with cerebral hemisphere expansion (Morris-Kay, 2001; Jiang et al., 2002; Schoch, 2014; Maddin et al., 2016; Blackburn et al., 2019; Lesciotto and Richtsmeier, 2019). However, to fully comprehend evolutionary patterns of the vertebrate skull, it is vital to investigate such relationships in diverse vertebrate groups and to understand the biochemical processes that underlie them.

The craniofacial skeleton is the most conspicuous part of the vertebrate skull, comprising the bones of the face, cranial vault and lower jaw. The great diversity seen in the craniofacial skeleton of vertebrates is the manifestation of multiple factors such as phylogeny, function, and development. However, there remains an incomplete understanding of all potential factors influencing craniofacial morphology and evolution. The bones of the craniofacial skeleton mostly develop from cranial neural crest $(\mathrm{CNC})$ cells that become chondrocytes of the cranial cartilages and osteoblasts of the craniofacial bones that undergo intramembranous ossification. The outgrowth of all CNC cells is controlled by several important morphogens - Sonic hedgehog (Shh), Fibroblast growth factor 8 (Fgf8), Chordin (Chd), and Noggin (Nog), to name a few (Richtsmeier and Flaherty, 2013). These morphogens are produced and released 
from signaling centers in the embryo to orchestrate normal development (Vieira et al., 2010). Previous experiments aimed at understanding the function of these morphogens yielded overlapping and similar results. Double knockdown of $C h d$ and Nog in mouse resulted in an underdeveloped forebrain, olfactory and optic cyclopia, and a complete lack of mandibular structures (i.e., agnathia; Bachiller et al., 2000). Inhibition of Shh expression in mouse and chick both resulted in cyclopia of the optic and olfactory system, as well as severely malformed facial bones (Chiang et al., 1996; Marcucio et al., 2005). Viral vector mediated inhibition of both $S h h$ and $F g f 8$ expression in chick led to subsequent malformations of beak structures (Abzhanov and Tabin, 2004). Nosip (Nitric Oxide Synthase-Interacting Protein), an underexplored gene involved in forebrain and facial development, has also seen similar results. Knockout of Nosip in mouse resulted in optic cyclopia, holoprosencephaly, and palatal disruption (Hoffmeister et al., 2014). While interruption of these forebrain-regulatory genes clearly has effects on optic, olfactory, and craniofacial structural development, each of the studies mentioned were conducted either in chick or mouse. If we can determine whether we would see the same correlations in an amphibian species, we may be able to conclude these patterns are conserved among tetrapods more broadly, and infer the ancestral condition for amphibians, as well as later-diverging vertebrates. Furthermore, establishing such a relationship will implicate the brain as playing a role in shaping the skull in amphibians, which is currently an unexplored aspect of skull evolution in the group.

One of the downfalls of genetic interruption of forebrain genes is that many of these genes have multiple roles in embryonic development, such as the additional role Shh and Fgf8 play in limb development (Richman and Tickle, 1992; Yang and Niswander, 1995; Mariani et al., 2008). This raises the issue of overlapping 
morphologies that may prove difficult to isolate the source of the manipulation. By physically removing the target tissue, specific morphogen sources in regions of interest can be reduced without disrupting its additional roles elsewhere in the embryo. A more specific method of gene interruption, where genes can be interrupted in specific tissue types, is conditional knockout (Tsien et al., 1996); however, this method has still not been thoroughly tested in $X$. laevis. Tissue ablation, and how it can identify and impact co-dependent structures, has regularly been used to study development and regeneration capabilities (Dufton et al., 2012). In one such study, the brains of NF stage $25 X$. laevis embryos were ablated and as a result it was found to be required for normal axial development, specifically for peripheral nervous system and muscle development (Herrera-Rincon et al., 2017).

As the neural tube begins to segment into the fore-, mid-, and hindbrain, three signaling centers begin to form - the midbrain-hindbrain boundary, zona limitans intrathalamica, and the anterior neural ridge (Houart et al., 1998; Millet et al., 1999; Zeltser et al., 2001). The latter of the three is of particular interest because it resides at the anterior-most point of the developing forebrain, which is our region of interest. This region of neuroepithelium produces and secretes fibroblast growth factor 8 (Fgf8), which as previously mentioned has a number of developmental roles in patterning the head (Houart et al., 1998; Vieira et al., 2010). There is also a high concentration of Shh in this region, which is required for medial patterning of the central nervous system (Abzhanov and Tabin, 2004; Vieira et al., 2010). By removing forebrain tissue and the neuroepithelial ANR, we can induce a targeted disruption of local Fgf8 and Shh expression to study how it will influence nearby tissues, specifically the chondrocranial portion of the skull, which acts as an initial guide for ossification patterns in multiple cranial structures by creating a primary scaffolding 
for dermal bones (Trueb and Hanken, 1992; Kawasaki and Richtsmeier, 2017; Pitirri et al., 2020). If this is found to be the case, we gain evidence of a shared developmental interaction between these factors in the brain and the adjacent skull structures.

The goal of this chapter is to study the role of the forebrain in the structural development of the chondrocranium. To ensure targeted disruption of local signals, the forebrain tissue was removed during the embryonic tailbud stage, after the neural tube closes and begins to segment into fore-, mid-, and hindbrain, and the specimens were left to continue to develop. The specimens were then fixed at tadpole stage to examine the resulting physical changes on the chondrocranium. The internal and external resulting morphologies are described here, and the potential role of signalling pathways and the impacts of their disruption is discussed. This study allows us to integrate our results based on an amphibian species with the current knowledge of brain-skull interactions derived from amniote models of mouse and chick to better understand this distribution of similarities and differences across tetrapods.

\section{2: Methods}

\subsection{1: Animal Care and Breeding}

A breeding colony of adult Xenopus laevis is maintained in the Maddin Lab at Carleton University, Ottawa, Canada (original source: Marine Biology Laboratories, Massachusetts). All X. laevis protocols used in this study are in accordance with the Canadian Council on Animal Care policies and Carleton University's Animal Care Committee animal use protocols (AUP \#102952 and \#103203). Spawning was induced by initially injecting a female with $50 \mathrm{IU}$ of human chorionic gonadotropin (HCG; Sigma C1063). Forty-eight hours later, the female received a second HCG 
injection of 500 IU. Concurrently, a male was injected with 50 IU of HCG. Upon completion of injections, both frogs were placed into one nuptial tank with tubing and artificial foliage. Embryos were collected the next morning and dejellied using a $2 \%$ buffered solution of L-cysteine free base (Fisher Scientific BP376100). Embryos were then left to develop in agar-lined petri dishes filled with autoclaved 10\% Holtfreter's solution at room temperature until NF stage 36 (Nieuwkoop and Faber, 1994). At this stage, they were transferred to tanks with $20 \%$ Holtfreter's solution. When tadpoles were able to feed they were fed Tropical Pro Defence Hi-Protein food once daily. All staging was conducted according to Nieuwkoop and Faber (1994).

\subsection{2: Microsurgery}

Xenopus laevis embryos, NF stages 22 to 28, were selected and placed into two groups: controls and forebrain removal $\left(\mathrm{FB}^{-}\right)$(Appendix $\mathrm{B}$ for surgery schematic). These stages were chosen as before NF stage 22, the neural tube has not fully segmented into the three-vesicle regions which makes it more difficult to remove the forebrain only without damaging the midbrain in the process. After NF stage 28, the embryos move quite a lot, making it difficult to remove the forebrain clearly without anesthetizing them. Embryos were placed in the fridge at $4^{\circ} \mathrm{C}$ for 20 minutes prior to surgery to slow movement. Surgery was performed in an agar-lined petri dish filled with autoclaved $10 \%$ Holtfreter's solution. Under a dissecting microscope, forebrain tissue was removed from $\mathrm{FB}^{-}$embryos, cutting from the cement gland to the forebrain-midbrain boundary, with the depth reaching the anterior border of the optic vesicles. This was done using a tungsten needle (Roboz Surgical, RS-6060, RS-6063). Optic vesicles were avoided during this removal to avoid the optic placode that resides within it. The paired olfactory placodes flank the forebrain and their location 
coincides with the location of the target tissue. Following forebrain surgery, embryos were left in the surgical dish to heal for 30 minutes (Fig. 3.1A), after which time they were transferred to a clean agar-lined petri dish filled with autoclaved $10 \%$ Holtfreter's solution. Surviving specimens were imaged at 24 hours post-surgery (Fig. 3.1B). Solutions were changed daily until NF stage 36 when they were then transferred to small tanks with $20 \%$ Holtfreter's solution and cared for according to standard procedures outlined in the above section.

\subsection{3: Staging and Fixation}

The $X$. laevis embryos and tadpoles were observed daily and were staged according to the table of Nieuwkoop and Faber (1994). Tadpoles were left to develop until at least until NF stage 41 . We note that $\mathrm{FB}^{-}$were only viable until approximately NF stage 45-50. As they approached these stages, they were anesthetized using a neutral-buffered, 4\% MS-222 solution (Sigma-Aldrich E10521, St. Louis, MO) and fixed overnight in 10\% neutral-buffered formalin (NBF, Thermo Fisher Scientific SF1004, Waltham, MA). After fixation, tadpoles were washed in distilled water and transferred through an ethanol dehydration series (approximately one hour in each: distilled water, $30 \%$ and $50 \%$ ) before final storage in $70 \%$ ethanol.

\subsection{4: Cartilage Staining}

To study morphological changes incurred by the forebrain surgical resection on $X$. laevis embryos, control and experimental specimens were cleared and stained for skeletal structure visualization. All specimens prior to staining were fixed in $10 \%$ NBF and stored at room temperature in $70 \%$ ethanol. Imaging for all stained 
specimens was conducted using Zeiss SteREO Discovery.V8 stereo microscope and Axio camera.

As survivorship of specimens was only up to stages where cartilage alone is present (i.e., approx. NF stage 51, Chapter 2), only a cartilage stain was performed on specimens here. For visualization of cartilage, specimens were placed in a solution of Alcian blue for up to six hours, time depending on specimen size (8GX, Acros Organics A3157) (20 mL acetic acid, $75 \mathrm{~mL}$ of $95 \%$ ethanol, $0.1 \mathrm{~g}$ alcian blue). Specimens were then rehydrated through an ethanol series (one hour in each: $70 \%$, $50 \%, 30 \%$, and distilled water). Following rehydration, specimens were placed in a solution of trypsin and saturated borate (30\% saturated borate, $1 \%$ trypsin) in a $37^{\circ} \mathrm{C}$ incubator until the specimen's tissue was soft and approximately $50 \%$ cleared. To finish clearing and prepare the specimen for long-term storage, specimens were moved through a $1 \% \mathrm{KOH}$ in glycerol series (approximately one hour in each: $3: 1$, 1:1, and $1: 31 \% \mathrm{KOH}$ to glycerol) for final storage in $100 \%$ glycerol.

\section{$\underline{3.3 \text { Results }}$}

Survival was obtained from specimens that had received surgeries conducted on NF stage 22 to 28 embryos. Earlier stages were attempted (NF stage 20/21); however, no survival was obtained. While NF stage 22 and NF stage 28 are morphologically quite different, the effects of forebrain removal did not vary significantly among specimens resulting from surgery at any of these stages. Regularly affected structures include the suprarostral plate, palatoquadrate and its various processes, Meckel's cartilage, infrarostral, and the structures surrounding the subocular foramina. Additionally, two non-skeletal structures, the olfactory capsules and eyes, were also commonly affected. No changes to the aforementioned structures 
are isolated to any one stage over the others. Three out of $35 \mathrm{FB}^{-}$embryos displayed a normal phenotype and their stages of their surgeries were NF stages 24, 25, and 27.

\subsection{1: 24-hour recovery anatomy}

To ensure the recovery of the forebrain-less $\left(\mathrm{FB}^{-}\right)$embryos, they were observed for 30 minutes immediately afterwards, as well as imaged 24 hours postsurgery (Fig. 3.1). Thirty minutes post-surgery, the $\mathrm{FB}^{-}$embryos showed contraction of surface ectodermal tissue around the resected site (Fig. 3.1A). This indicated that the healing process had begun and once it was mostly covered in surface ectoderm, embryos were left to continue developing. Twenty-four hours later, the site had completely healed over and there was an indent where the forebrain would ordinarily be found (Fig. 3.1B). Most $\mathrm{FB}^{-}$embryos were around NF stage 33-36 during this timeframe. The cement gland and optic vesicle remained unaffected; if the embryos showed signs of damage to either of these structures, they were removed from the $\mathrm{FB}^{-}$ group to avoid introducing additional variables that might affect chondrocranial development. Other physical changes were commonly observed among $\mathrm{FB}^{-}$embryos, such as indentations on the tailfin. Where it is usually straight or slightly curved, some $\mathrm{FB}^{-}$embryos showed bumpy tailfins (Fig. 3.1B). While this thesis does not discuss the specific changes to axial development following forebrain removal, HerreraRincon et al. (2017) provide insights into how the brain is essential for normal development in other parts of the animal, such as axial muscle and peripheral nerve development. 


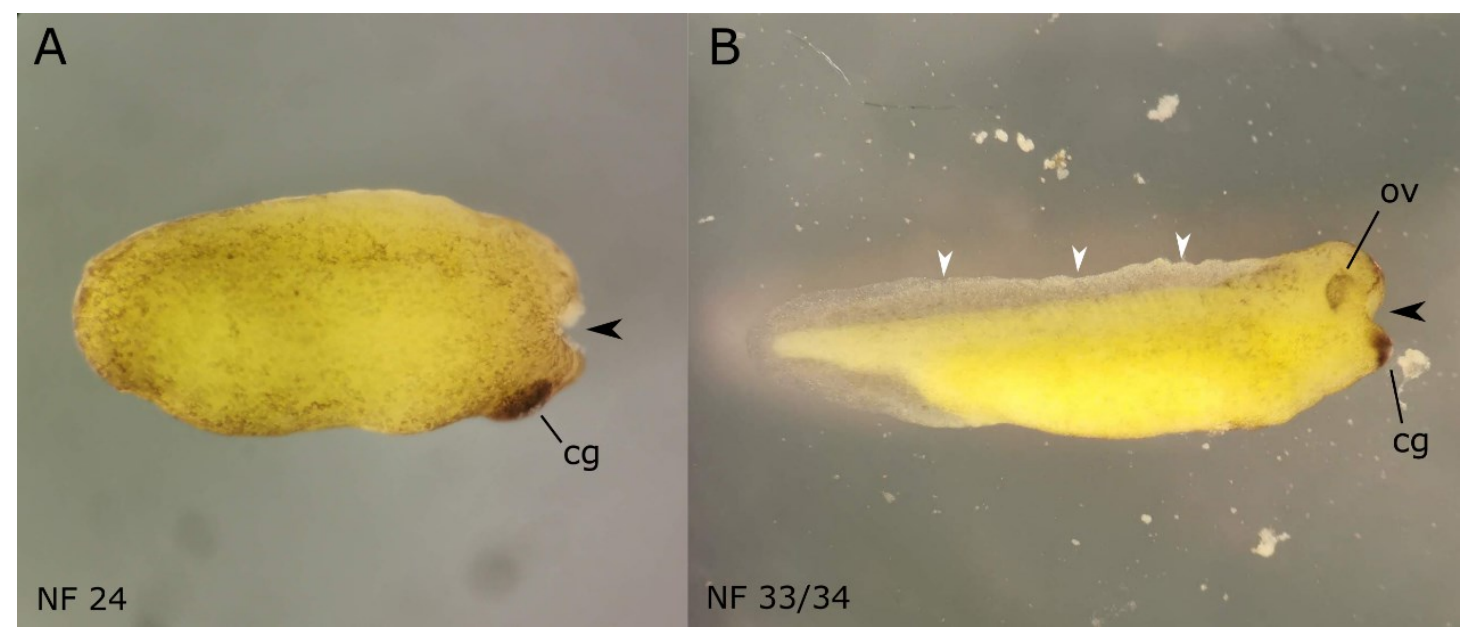

Figure 3.1. Xenopus laevis embryo surgery location and $24 \mathrm{hr}$ recovery. A, NF stage 24 embryo 30 minutes post-surgery, lateral view. Thirty minutes after forebrain microsurgery, the cement gland is still intact and the dorsal skin of the embryo is contracting around the surgery site (indicated by black arrow). B, NF stage 33/34 embryo 24 hours post-surgery, lateral view. The cement gland and optic vesicle are still visible. There is an indentation on the anterior end of the embryo where the forebrain was removed (indicated by black arrow). Developing tailfin has notable ridges that are not observed in normal embryos (white arrows) (Nieuwkoop and Faber, 1994). Abbreviations: cg, cement gland; ov, optic vesicle.

\subsection{2: External features post-surgery}

Before the $\mathrm{FB}^{-}$tadpoles were cleared and stained, they were examined for external changes. Two external anatomical structures were affected the most: the eyes and the nasal pits. The eyes showed variable severity in malformation. Some specimens exhibited normal development; the eyes migrated to the correct region and showed all necessary structures, such as the lens and a normal retinal pigmented epithelium (Fig. 3.2A). Five showed lateral migration of the eyes but with an abnormal conical rather than spherical shape. Eight showed no clear outline of the retinal pigmented epithelium, five of these overlapping with the conical shaped morphology (Fig. 3.2H). The pattern of pigmentation was inconsistent, and the eyes were transparent in certain areas. When the $\mathrm{FB}^{-}$specimens were moved through the Alcian blue staining series, the abnormally developed eyes began to disintegrate or pull away from the rest of the tissue (Fig. 3.2G-I). 
In one specimen, only one eye developed (Fig. 3.2C). The region to where the eye usually would migrate was defined by a protrusion with epithelial pigmentation but on the left side no eye developed and on the right side, the eye was much more anteriorly placed than expected. Where the eye usually sits is immediately lateral to the subocular foramen, anterior to the ventrolateral process of the palatoquadrate. In this specimen, however (Fig. 3.2C), the eye was instead overlying the anterior palatoquadrate region (near the pars articularis). Two specimens also exhibited severe optic cyclopia. In both specimens, the eyes were still connected to the midline by a poorly developed optic tract rather than fully separated and connected to the forebrain via only the optic nerves (Fig. 3.2B). The eyes are very close to the brain rather than laterally separated and the optic tract is covered in pigment. The left eye shows some internal organization, where an underdeveloped lens and a defined outer layer with the retinal pigmented epithelium can be seen. The right eye shows no internal organization, meaning no discernable pigmented retinal epithelium or lens. This also corresponds with the underdeveloped forebrain seen in the cyclopic tadpoles, as the anterior edge of the forebrain tapers and merges with the optic tract, rather than rounded at its anteriormost extent.

As with the eyes, severity of nasal pit malformation varied among $\mathrm{FB}^{-}$ specimens. Four specimens showed normal development of the nasal pits (Fig. 3.2A), whereas others showed mostly normal development on one side but had slightly to severely underdeveloped olfactory epithelium on the other side (Fig. 3.2D, G). In one $\mathrm{FB}^{-}$tadpole, we saw the same normal development on one side with the other side showing no signs of nasal pit development at all (Fig. 3.2F). There was no indication of olfactory epithelium development or pigmentation around the future nasal pit. 


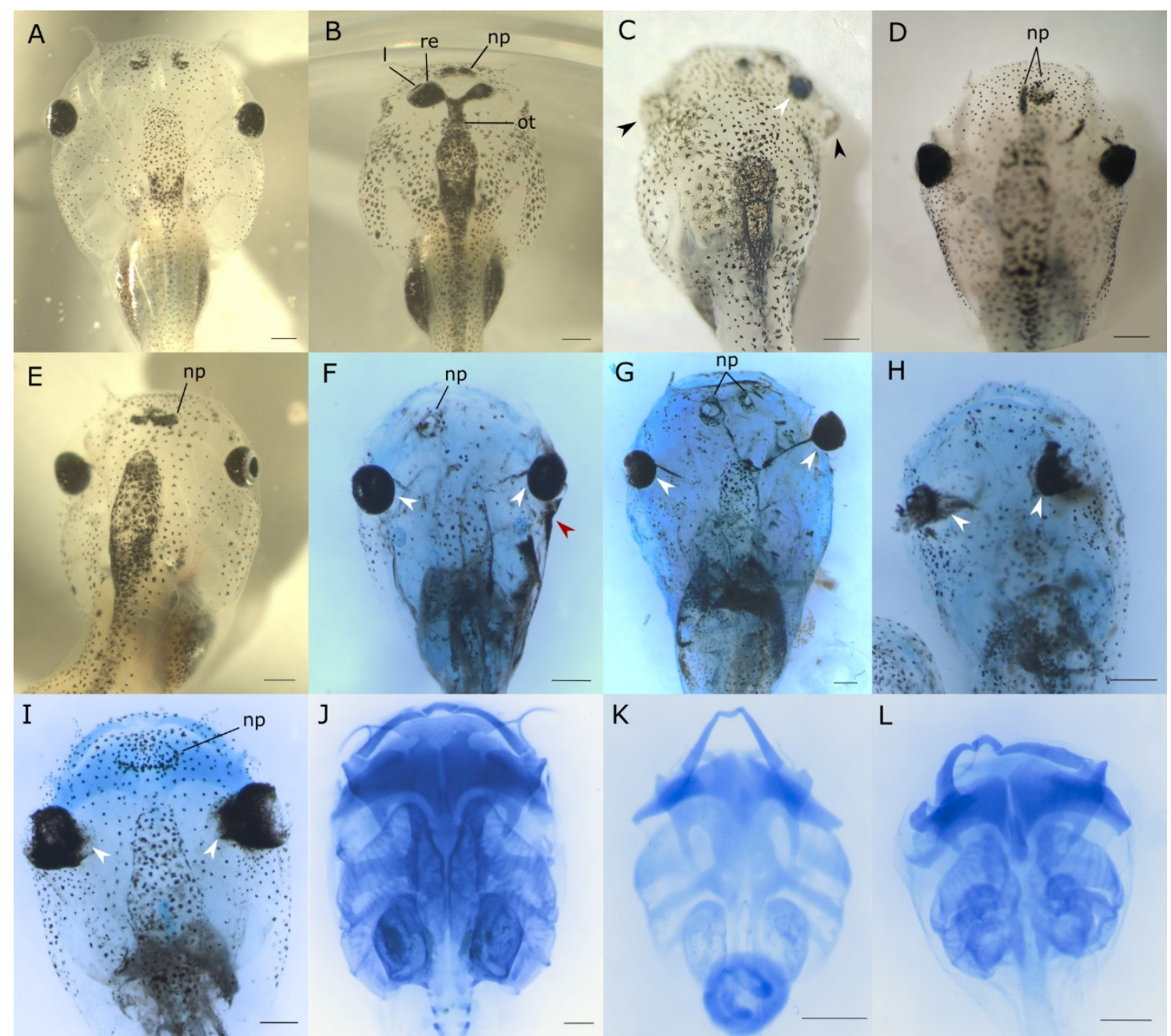

Figure 3.2. External/internal features and gross morphology of $\mathrm{FB}^{-}$specimens.

All specimens are in dorsal view. A-E, external features only. F-I, unbleached Alcian Blue stained tadpoles. J-L, bleached Alcian Blue stained tadpoles. Various malformations observed in the post-surgery tadpoles include olfactory/optic cyclopia, lack of internal eye organization, irregular epithelial pigmentation, and bilateral asymmetry. A, $\mathrm{FB}^{-}$tadpole with normal morphology post-surgery. $\mathrm{B}, \mathrm{FB}^{-}$tadpole with olfactory/optic cyclopia. The left eye shows some internal organization and the optic tract is hyperpigmented. $\mathrm{C}, \mathrm{FB}^{-}$tadpole displaying missing left eye and misplaced right eye (white arrow), protrusions where they are normally positioned are indicated by black arrows. Olfactory cyclopia is also observed. $\mathrm{D}, \mathrm{FB}^{-}$tadpole with underdeveloped left nasal pit and narrowing of maxillary/mandibular region. $\mathrm{E}, \mathrm{FB}^{-}$ tadpole with olfactory cyclopia. $\mathrm{F}, \mathrm{FB}^{-}$tadpole with missing right nasal pit, no internal eye organization (white arrows), and uneven distribution of pigmentation (red arrow). $\mathrm{G}, \mathrm{FB}^{-}$tadpole with underdeveloped right nasal pit and no internal eye organization (white arrows). $\mathrm{H}, \mathrm{FB}^{-}$tadpole with severely underdeveloped, conical shaped eyes that show no internal organization (white arrows). $\mathrm{I} \mathrm{FB}^{-}$tadpole with olfactory cyclopia and eyes with no internal organization. $\mathrm{J} \mathrm{FB}^{-}$tadpole with normal chondrocranial development. $\mathrm{K}-\mathrm{L}, \mathrm{FB}^{-}$tadpoles with malformed anterior chondrocranial elements, posterior region remains unaffected. Abbreviations: 1, lens; $\mathrm{np}$, nasal pit(s); ot, optic tract; re, retinal epithelium. Scale bars equal to $500 \mu \mathrm{m}$. 
In nine specimens (Fig. 3.2B, E, I), the nasal pits failed to separate into two structures and instead formed one large opening at the anterior end of the head. This single pocket contained two olfactory epithelia but failed to separate along the midline. In one specimen (Fig. 3.2H), no signs of nasal development for either the nasal pits or olfactory epithelium were observed. There were also signs of reduced forebrain size in multiple specimens (Fig. 3.2B, C, F, H, I) - this was determined by visual analysis and comparing its position relative to nearby structures.

\subsection{3: Effects of forebrain removal on chondrocranial development}

Without the forebrain, most FB- tadpoles exhibited structural changes to the chondrocranium. A number of changes to the gross morphology of the tadpole chondrocrania were observed, and the remaining changes that were observed can be grouped into four main categories: palatal and rostral, mandibular, hyoid arch, and subocular developmental interference.

\section{Gross morphological changes}

In one $\mathrm{FB}^{-}$tadpole, the anterior half of the chondrocranium was significantly less wide than the posterior half (Fig. 3.2K). The posterior chondrocranium, including the ceratobranchial elements, the parachordal cartilage, and the otic capsules, showed a normal morphology with regards to width; however, the anterior chondrocranium was overall reduced in width, in addition to the specific morphological changes in any given structure. All elements that typically reach quite far laterally, including the muscular process of the palatoquadrate and the ceratohyals, were shortened along this axis. This same $\mathrm{FB}^{-}$tadpole also shows an interesting mandibular morphology where even with the dorsal chondrocranium width reduction, Meckel's cartilage still reached 
a relatively normal length between its articulation points with the infrarostral and palatoquadrate. Meckel's cartilage and the infrarostral now appear to be V-shaped, however, rather than the gently curved shape they normally exhibit. Certain elements were also observed to be shifted in their position relative to the surrounding structures, such as the suprarostral plate being more anterior than the infrarostral. The shape of the structures was not always altered.

Another result to note is that several structures showed no morphological changes following forebrain removal surgery. Consistently, unaffected structures were all posterior to the ascending process of the palatoquadrate: the otic capsules, parachordal cartilage, occipital arch, plus the ceratobranchial elements. All affected structures were restricted to those located anterior to this region.

\section{Palatal and rostral development}

Differences in the morphology of the palatal and rostral structures were observed between the $\mathrm{FB}^{-}$embryos and control embryos; specifically in the suprarostral plate, palatoquadrate (muscular process and larval pars articularis), tentacular cartilage, and the anterior portion of the frontoparietal fontanelle. The shape of the nasal foramina is dependent on the surrounding structural elements, bordered medially by the suprarostral plate, anterolaterally by the tentacular cartilage, and posteriorly by the palatoquadrate. Any changes to any of these structures may alter the shape of the nasal foramina. In one specimen, the nasal foramina were bilaterally uneven, with the left foramen reaching further inward medially than the right (Fig. 3.3A). This was caused by a misshapen suprarostral plate, where the left side was more deeply notched than the right. Another specimen showed a suprarostral plate divided along the midline, with lateral edges curving posteriorly (Fig. 3.3B). 


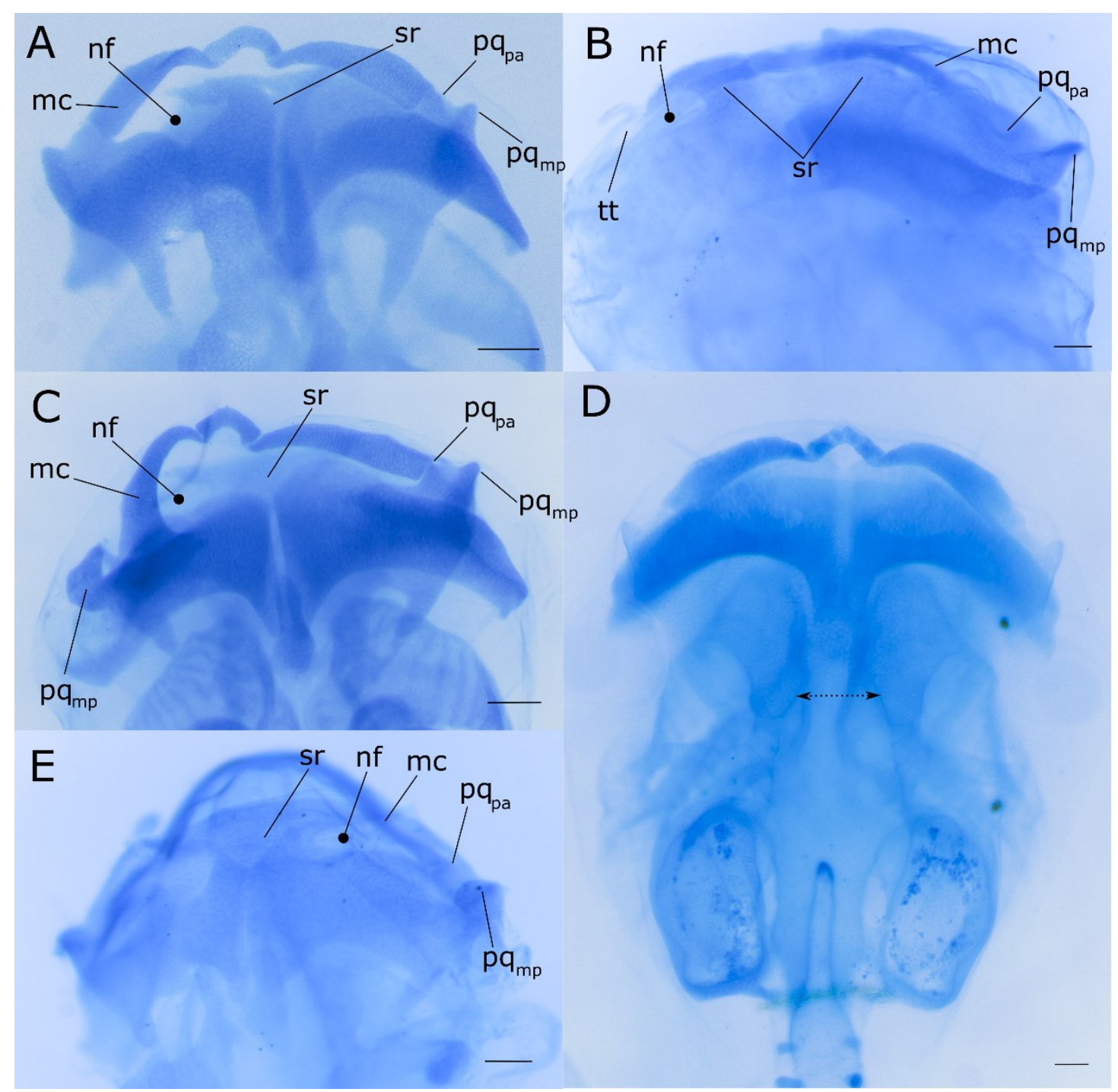

Figure 3.3. Palatal and rostral development of $\mathbf{F B}^{-}$specimens. Various malformations observed in the palatal/rostral regions are seen in the suprarostral plate, nasal foramina, and anterior palatoquadrate structures. All specimens are in dorsal view. $\mathrm{A}, \mathrm{FB}^{-}$tadpole with malformed suprarostral plate resulting in uneven nasal foramina. $\mathrm{B}, \mathrm{FB}^{-}$tadpole with suprarostral plate divided along the midline, separating it into two structures and resulting in uneven nasal foramina, underdeveloped tentacular cartilage, and a more laterally-oriented muscular process of the palatoquadrate. $\mathrm{C}, \mathrm{FB}^{-}$tadpole with underdeveloped left anterior palatoquadrate structures, resulting in poor articulation with Meckel's cartilage. $\mathrm{D}, \mathrm{FB}^{-}$tadpole with narrowing of the anterior frontoparietal fontanelle in response to reduced forebrain size. $\mathrm{E}, \mathrm{FB}^{-}$tadpole with underdeveloped suprarostral plate and a smaller right nasal foramen. Muscular process of the palatoquadrate is curling inward medially. Abbreviations: mc, Meckel's cartilage; nf, nasal foramen; $\mathrm{pq}_{\mathrm{mp}}$, muscular process of the palatoquadrate; $\mathrm{pq}_{\mathrm{pa}}$, larval pars articularis of the palatoquadrate; sr, suprarostral plate; tt, tentacular cartilage. Scale bars equal to $200 \mu \mathrm{m}$. 
This same specimen also had an underdeveloped tentacular cartilage. Misshapen, uneven nasal foramina were seen in about one third of the $\mathrm{FB}^{-}$specimens; however, all specimens retained paired nasal foramina.

The anterior palatoquadrate exhibited considerable changes in morphology in some specimens. In one specimen (Fig. 3.3C), the right anterior palatoquadrate exhibited a normal shape but the left side did not resemble its normal shape. It did not articulate with Meckel's cartilage, and it lacked both the muscular process and the pars articularis of the palatoquadrate. Other specimens showed mild

underdevelopment with less prominent muscular processes or the lateral most part of the palatoquadrate curled in on itself medially (Fig. 3.3E).

The anterior margin of the frontoparietal fontanelle showed minor changes. In one specimen (Fig. 3.3D), rather than having a rounded anterior margin (as seen in Fig. 2.2 D-F), the fontanelle margin was tapered.

\section{Mandibular development}

Effects on mandibular morphology are concentrated in two structures - the infrarostral and Meckel's cartilage - and how they articulate with each other and the surrounding structures. The mandibular structures were the most consistently affected structures following forebrain removal, showing alterations in 25 of $38 \mathrm{FB}^{-}$tadpoles. The infrarostral, the anterior most element, is a small, median, unpaired structure that articulates with Meckel's cartilage on either side laterally. It is slightly curved posteriorly, which is a feature we saw the most variation in. The infrarostral cartilage tends to become less curved as development proceeds and it eventually fuses laterally with Meckel's cartilage on either side (Trueb and Hanken, 1992). However, ten specimens did not show the expected curvature of this element in comparison to that 


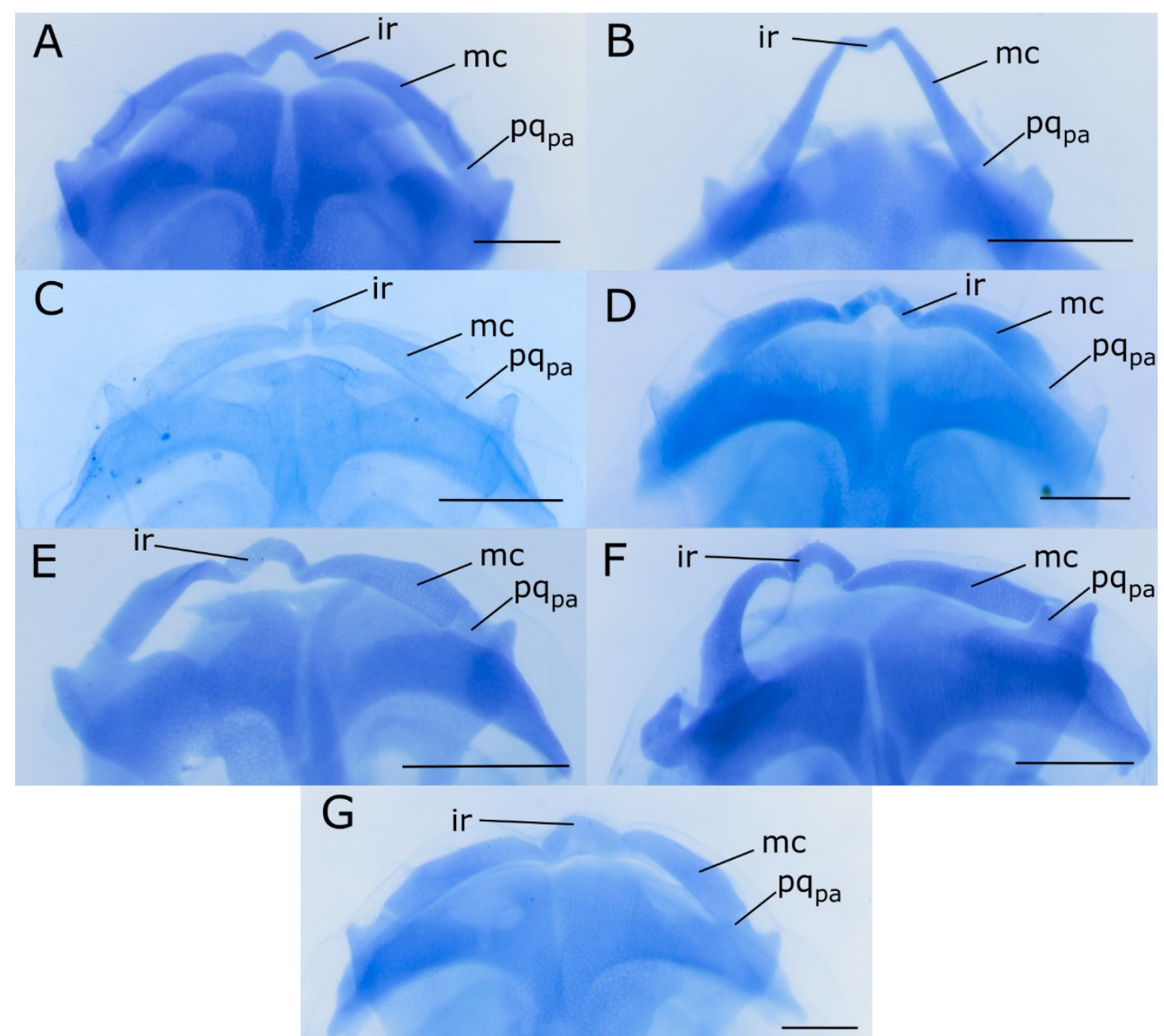

Figure 3.4. Mandibular development of $\mathrm{FB}^{-}$specimens. Various malformations of the mandibular region observed in the infrarostral, Meckel's cartilage, and the larval pars articularis of the palatoquadrate. All specimens are in dorsal view. A, $\mathrm{FB}^{-}$ tadpole with increased bending in the infrarostral. $\mathrm{B}, \mathrm{FB}^{-}$tadpole with an inverted infrarostral and an anterior shift of all mandibular elements. $\mathrm{C}, \mathrm{FB}^{-}$tadpole with thickening of Meckel's cartilage and a large increase in infrarostral curvature. D, $\mathrm{FB}^{-}$ tadpole with thickening of Meckel's cartilage. E, $\mathrm{FB}^{-}$tadpole with bilateral asymmetry in mandibular elements. $\mathrm{F}, \mathrm{FB}^{-}$tadpole with an underdeveloped left half of Meckel's cartilage, resulting in poor articulation with the larval pars articularis of the palatoquadrate. $\mathrm{G}, \mathrm{FB}^{-}$tadpole with bilateral asymmetry in the mandibular elements. Abbreviations: ir, infrarostral; mc, Meckel's cartilage, $\mathrm{pq}_{\mathrm{pa}}$, larval pars articularis of the palatoquadrate. Scale bars equal to $500 \mu \mathrm{m}$. 
of control specimens at the respective stages. In those specimens, the infrarostral was more curved than that of a normal specimen of the same stage (Fig. 3.4A). One $\mathrm{FB}^{-}$ tadpole displayed an inverted curvature, with the concavity of the curve oriented anteriorly (Fig. 3.4B). Another showed the infrarostral highly bent into a horseshoe shape instead of the slight curve of a control specimen (Fig. 3.4C).

Meckel's cartilage also showed changes in morphology and displayed asymmetrical alterations. Increased curvature of Meckel's cartilage was seen in twelve $\mathrm{FB}^{-}$specimens, bilaterally, and five $\mathrm{FB}^{-}$specimens, unilaterally. In some specimens, Meckel's cartilage had thickened slightly and was more curved on the medial side, although it articulated normally with the palatoquadrate and the infrarostral (Fig. 3.4D, E). As for asymmetrical changes, some showed more severe asymmetry (Fig. 3.4F, G) with Meckel's cartilage no longer articulated well with the palatoquadrate (Fig. 3.4F). In one specimen (Fig. 3.4B), Meckel's cartilage was oriented more anteroposteriorly, and the entire mandible showed a pronounced anterior shift.

\section{Hyoid arch development}

Of the hyobranchial cartilages, the ceratobranchial elements remained unaffected by forebrain removal. The only changes seen were in the paired ceratohyals and the basihyobranchial. The changes seen in these elements were slight compared to other morphological changes. In one specimen, the ceratohyals were much closer together along the midline, which displaced the basihyobranchial element into a further posterior position than in a control specimen (Fig. 3.5A). The ceratohyals of the same specimen were also pointed more laterally rather than posteriorly, creating much more space between the ceratohyals and the planum 


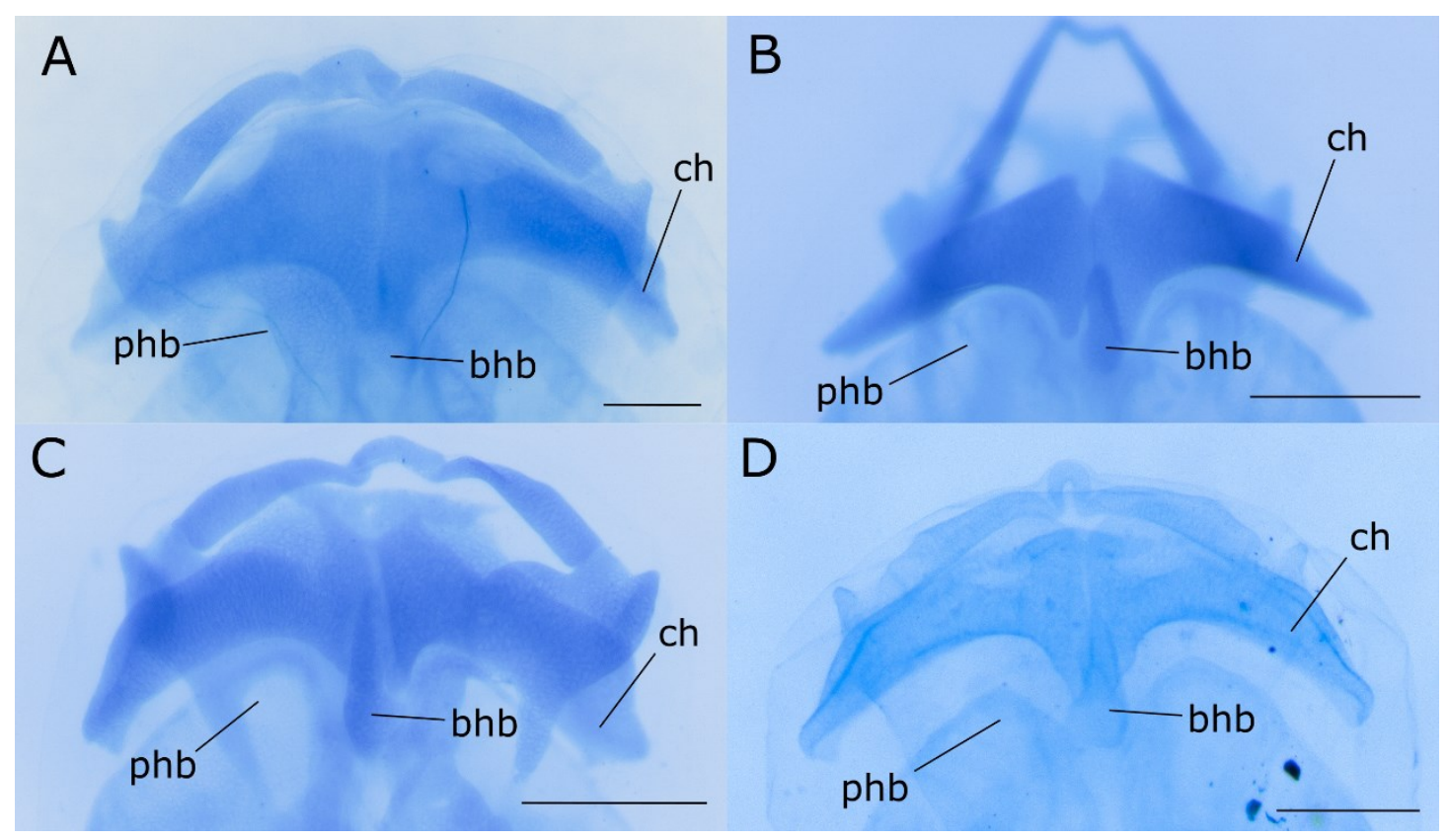

Figure 3.5. Development of the hyoid arch in $\mathbf{F B}^{-}$specimens. Various malformations of the hyoid arch were observed in the basihyobranchial and the ceratohyals. All specimens are in ventral view. $\mathrm{A}, \mathrm{FB}^{-}$tadpole with more medially shifted ceratohyals, displacing the basihyobranchial more posteriorly. $\mathrm{B}, \mathrm{FB}^{-}$tadpole with more medially shifted ceratohyals. Left ceratohyal is more anteriorly oriented, increasing space between the ceratohyal and the planum hyobranchial. $\mathrm{C}$ and $\mathrm{D}, \mathrm{FB}^{-}$ tadpoles with more angular lateral edge of ceratohyals, increasing space between the ceratohyal and the planum hyobranchial. Abbreviations: bhb, basihyobranchial; ch, ceratohyal; phb, planum hyobranchial. Scale bars equal to $500 \mu \mathrm{m}$.

hyobranchial. This morphology was seen in several other specimens (Fig. 3.5B-D). The ceratohyals, which usually appear as posteriorly oriented, broad plates, also appeared to be pointed on the lateral edges in some $\mathrm{FB}^{-}$tadpoles (Fig. 3.5B-D).

\section{$\underline{\text { Subocular cartilage/foramina development }}$}

The subocular foramen is bordered medially by the trabecular cartilage, anteriorly by the palatoquadrate, laterally by the subocular cartilage, the latter of which eventually meets the ascending process of the palatoquadrate bordering the foramen posteriorly. The shape of the foramen in $\mathrm{FB}^{-}$specimens changed depending on the nature of the shape of development in these surrounding structures. In one 
specimen (Fig. 3.6A), the subocular cartilage on both sides of the chondrocranium was curled medially at the fusion point with the ascending process of the palatoquadrate. The anterior protrusion of the ascending process was also curved medially, rather than growing directly to meet the subocular cartilage. I also observed that some foramina are unilaterally malformed. In another specimen (Fig. 3.6B), the left subocular foramen was notably more posteriorly located than the right. In a specimen of normal development at this stage, the middle of the subocular foramen is typically aligned with the anteriormost margin of the frontoparietal fontanelle; instead, in the $\mathrm{FB}^{-}$specimen observed here, the anterior edge of the foramen was in line with the anterior most margin of the frontoparietal fontanelle. In another $\mathrm{FB}^{-}$ specimen, the edges of the left subocular foramen were not smooth and rounded (Fig. 3.6C), as in a control specimen. The more jagged edges of the foramen observed in this tadpole were seen on only the left side.

The ventrolateral and ascending processes of the palatoquadrate were elements that also exhibited great variability and morphological changes as a result of forebrain removal. The ascending process usually has a gentle ventrolateral slope. We can see how the morphology changes in two $\mathrm{FB}^{-}$tadpoles (e.g., Fig. 3.6C and Fig. 3.7B), where the ascending process on the left half of the chondrocranium stopped abruptly and grew ventrally before growing back out laterally. Another feature seen in three $\mathrm{FB}^{-}$tadpoles was the lack of complete lateral extension of the ventrolateral process of the palatoquadrate. Under normal circumstances, this process of the palatoquadrate would grow laterally and then develop an anteroposterior protrusion. Instead, the cartilage formed a ball-like structure and curled medially back in on itself (Fig. 3.7A). 


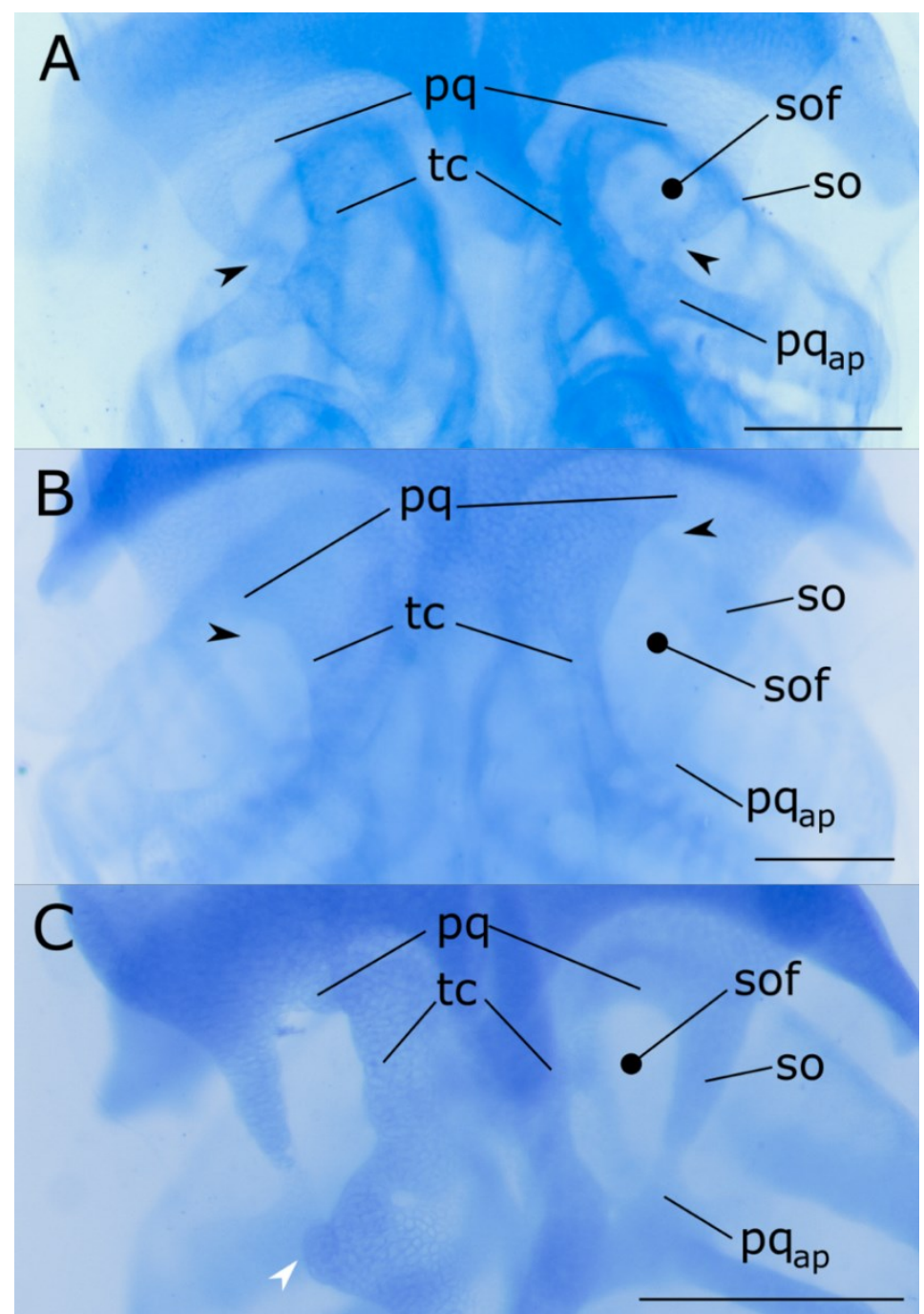

Figure 3.6. Development of the subocular structures in $\mathbf{F B}^{-}$specimens. Various malformations of the subocular structures observed in the subocular cartilage, tentacular cartilage, and the ascending process of the palatoquadrate resulting in malformed subocular foramina. All specimens are in dorsal view. A, Subocular cartilage and ascending process of the palatoquadrate curl inward medially as they join (black arrows), changing the shape and reducing the size of the subocular foramina. B, Subocular foramina are bilaterally uneven with the right foramen occurring farther anteriorly than the left foramen (black arrows). Their posterior most points are not uneven. The left subocular foramen is smaller than the right subocular foramen. C, Border of left subocular foramen has jagged edges rather than the smooth borders seen on the right. The left ascending process of the palatoquadrate is misshapen and abruptly extends ventrally before further extending laterally (white arrow). Abbreviations: $\mathrm{pq}$, palatoquadrate; $\mathrm{pq}_{\mathrm{ap}}$, ascending process of the palatoquadrate; so, subocular cartilage; sof, subocular foramen; tc, trabecular cartilage. Scale bars equal to $500 \mu \mathrm{m}$. 


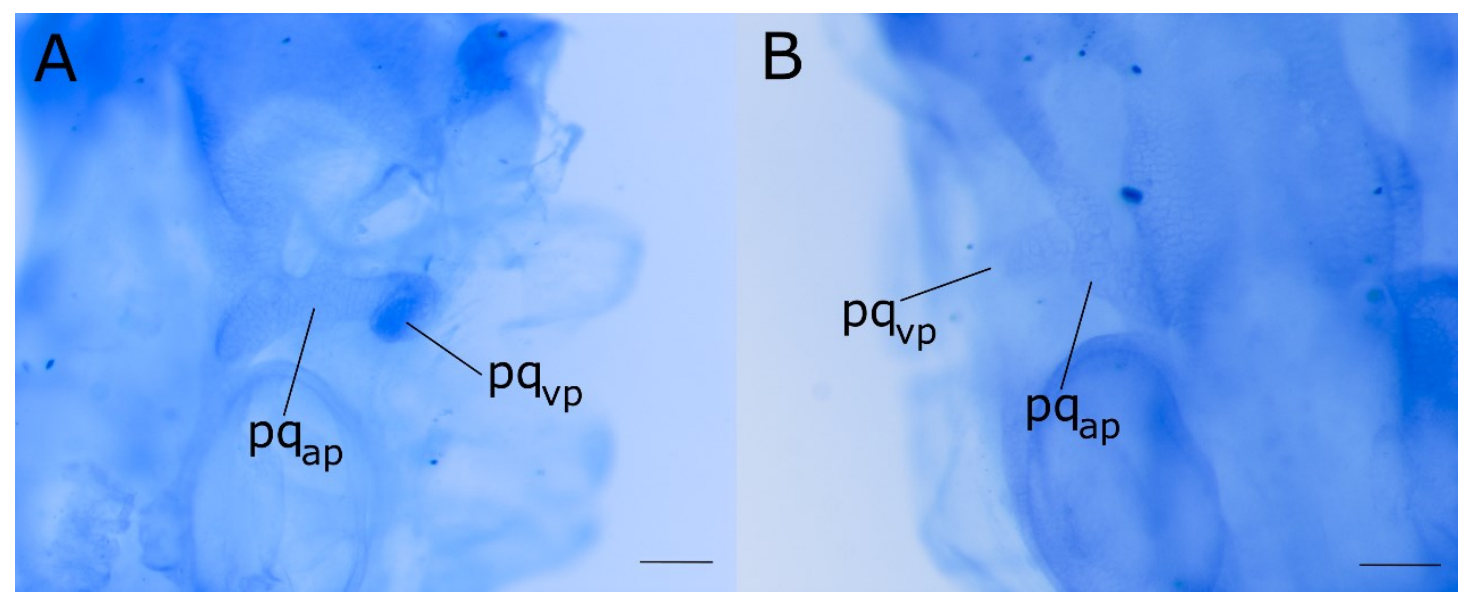

Figure 3.7. Development of the ventrolateral and ascending processes of the palatoquadrate in $\mathbf{F B}^{-}$specimens. Both specimens in dorsal view. A, Ventrolateral process of the palatoquadrate curls in on itself medially, forming a ball-like structure. $\mathrm{B}$, Ascending process of the palatoquadrate is misshapen and abruptly extends ventrally before further extending laterally. The ventrolateral process of the palatoquadrate is underdeveloped. Abbreviations: $\mathrm{pq}_{\mathrm{ap}}$, ascending process of the palatoquadrate; $\mathrm{pq}_{\mathrm{vp}}$, ventrolateral process of the palatoquadrate. Scale bars equal to $200 \mu \mathrm{m}$.

\section{4: Discussion}

With the removal of the forebrain during the embryonic tailbud stage, various anterior head structures were affected - specifically, the craniofacial elements. The observed results allow us to gain insight into the role in which the forebrain, and its surrounding structures, influence the development of major structural elements of the cranium. An interesting outcome observed here were impacts forebrain removal had on sensory structure development. This result reveals additional downstream effects of forebrain removal, i.e. effects on neural and sensory structures.

\subsection{1: Cyclopia and Shh expression}

Eye and nasal pit development were most consistently affected in specimens that had the forebrain removed. In many specimens, the eyes did not fully migrate to 
their lateral positions or were severely underdeveloped. In one specimen, the eyes were positioned between the anteriormost point of the brain and the nasal pits, unable to migrate laterally and were mostly attached to the optic tract along the midline. The nasal pits were even more variable in morphology. Missing and poorly migrated nasal pits as well as cyclopic features, where one single nasal pit contains both olfactory epithelia, were observed. These results suggest forebrain removal induced a form of holoprosencephaly that exhibits cyclopic characteristics (Heavner and Pevny, 2012).

Such cyclopia can be induced in several ways. One of the most well-known pathways that regulates telencephalic division is the sonic hedgehog signaling pathway. During knock-out experiments with targeted disruption of Shh in mouse, the cephalic neural tube did not have a distinct midline and displayed a single optic vesicle on the ventral midline (Chiang et al., 1996). Shh is a gene that is also present in $X$. laevis and has similar functions as in other models, such as in limb patterning (Endo et al., 1997) and dorsal midline development (Peyrot et al., 2011). Given the cyclopic features observed here, such as incomplete separation of the optic nerve and combined olfactory organs, a potential candidate source of such features in our $\mathrm{FB}^{-}$ specimens could be Shh disruption. Shh is secreted from the notochord and creates a ventral gradient throughout the neural tube, which counters BMP signaling (Roelink et al., 1994; Ekker et al., 1995; Liem et al., 2000). In removing the forebrain segment of the neural tube, it is possible that there was a minor decrease in $S h h$ expression in this anterior region. Since Shh expression wouldn't be completely eliminated due to its expression in other local tissues, such as the notochord and prechordal plate (Kiecker and Lumsden, 2005), we would not necessarily see a complete lack of midline formation but rather incomplete separation of bilateral sensory organs, such as we saw in two specimens (Fig. 3.2B). The anterior boundary of the prechordal 
plate and notochord in Xenopus reaches anteriorly to the forebrain-midbrain boundary (Illes et al., 2009). Since $S h h$ is expressed in the notochord as the morphogenetic signal can travel to the ANR, removing the forebrain does not eliminate all Shh signal in that region as the signal from the notochord may compensate (Kiecker and Lumsden, 2005). A similar phenotype is seen when $X$. laevis embryos were exposed to glyphosate-based herbicides and found that it caused craniofacial and optic alterations (Paganelli et al. 2010; Bonfanti et al., 2018). Paganelli et al. (2010) hypothesized that glyphosate increased endogenous retinoic acid (RA) concentrations, which subsequently caused a decrease in Shh signaling, further supporting the link between Shh and hemisphere division.

Not only does $S h h$ play a role in the creation of a sensory midline, but it is also needed in establishing internal eye structure. As $S h h$ is a ventralizing agent, it prevents the eye from becoming dorsalized by BMP4 expression in the dorsal region of the optic cup (Adler and Canto-Soler, 2007). Interestingly, in addition to the specimens that displayed a lack of a midline, we also saw those that do not have properly formed eyes. As it is known that coordinated cross-regulation of $\operatorname{Sh} h, F g f 8$, and Bmp4 expression is required to achieve normal eye development (Ohkubo et al., 2002), the observed phenotypes of the eyes further support a disruption to Shh signalling in $\mathrm{FB}^{-}$specimens. The observed changes to eye structure and development are discussed further below.

\subsection{2: Fgf8 expression and the removal of the ANR}

During certain developmental stages, at the anteriormost point of the developing brain, one can find the anterior neural ridge (ANR). The ANR is a local organizer responsible for patterning the telencephalic region of the forebrain in 
vertebrates and was first described in zebrafish (Houart et al., 1998). A characteristic morphogen of this region is $F g f 8$, a critical gene in the regulation of embryological development, as it in turn controls the expression of many other genes (Vieira et al., 2010; Pownall and Isaacs, 2010). Removal of the ANR region during early forebrain patterning is suggested to have a cascade of effects, such as the expansion of posterior forebrain regions, due to the downregulation of $F g f 8$ (Houart et al., 1998). The ANR, while critical for regionalization of the brain, also has a role in establishing the cartilaginous scaffolding for the development of the vertebrate head - i.e., the chondrocranium. The ANR controls this through regulation of $F g f 8$ expression, which in turn plays a role in chondrocyte differentiation from cranial neural crest (CNC) cells, also known as the epithelial to mesenchymal transition (Abzhanov and Tabin, 2004; Kalluri and Weinberg, 2009).

Epithelial-mesenchymal interactions are an integral part of the vertebrate skull development (Richman and Tickle, 1992). Reciprocal signaling involving Fgf8 expression between the dorsal neural tube and the facial primordia plays a crucial role in craniofacial development (Couly and Le Douarin, 1988; Anderson, 1997; Creuzet et al., 2004). CNC cells delaminate from the dorsal neural tube and migrate to the craniofacial primordia, as well as to the pharyngeal arches. Although it is CNC cells that give rise to the cartilaginous craniofacial structures, the forebrain provides important information to conduct the development of these structural elements (Anderson, 1997). This information comes in the form of fibroblast growth factors, notably $F g f 8$, from the ANR. In our study, removal of the forebrain during the embryonic tailbud stage in $X$. laevis likely resulted in the removal of the ANR, ultimately leading to reduced $F g f 8$ expression and potentially interfering with chondrification. 
As noted above, many studies have shown that holoprosencephaly may be induced through Shh inhibition (Chiang et al., 1996; Roessler et al., 1997; Cordero et al., 2004); however, it is also known that $S h h$, paired with $F g f 8$ expression, plays an important role in cartilage outgrowth (Eberhart et al., 2006; Hu and Marcucio, 2009). Trumpp et al. (1999) studied the role of Fgf8 signaling in CNC development. It was found that Fgf8 signaling from the ANR, in conjunction with Fgf2, is required to promote CNC survival and differentiation (Trumpp et al., 1999). For CNC cells to become cartilage, they must undergo chondrogenic differentiation. Abzhanov and Tabin (2004) studied the role of $S h h$ and $F g f 8$ expression on CNC migration and chondrogenesis in chick cranial development. They found that at zones where Fgf8 and Shh signals overlap is where CNC chondrogenic differentiation occurs. Shh signaling alone could not induce chondrogenesis but when combined with Fgf8 signals from the ANR was able to increase rates of chondrogenic differentiation (Abzhanov and Tabin, 2004). This establishes that Fgf8 signaling is needed for CNC survival, but Shh and Fgf8 are needed in combination to conduct CNC migration and chondrogenic differentiation. If $F g f 8$ expression is inhibited, cell death in the first branchial arch can occur, leading to missing chondrocranial and dermatocranial elements of the jaws (Trumpp et al., 1999). This may provide an explanation for the abnormal mandible and palatal structures observed here (Figs. 8 and 9).

With the removal of the forebrain, and thus the ANR, Fgf8 signaling is likely to be greatly reduced, which could have impacted the ability for the cartilage to grow to its final position. This can be seen in some specimens (e.g., Fig. 3.7) where the ventrolateral process of the palatoquadrate, a structure that usually has an anteroposterior process, instead shows the lateral most point of the ventrolateral process has curled in on itself. The inability for the cartilage to complete growth to its 
final destination is possibly due to reduced $F g f 8$ signaling (Anderson, 1997; Eagleson and Dempewolf, 2002). Although the exact role of $S h h$ and $F g f 8$ expression in chondrogenesis has not yet been determined in Xenopus, it is known that both Shh and $F g f 8$ are expressed in the anterior domain of the head and interruption of their expression can lead to craniofacial abnormalities (Chen et al., 2017). As Fgf8 and Shh signaling has been thoroughly studied in chick and mouse, it will be important to determine what similarities are shared by amphibians.

In the post-surgical specimens, we can observe the morphological changes in the chondrocranium caused by forebrain manipulation. All required cartilage elements are present in the head; however, they do not always display the normal morphology. One concern for the surgical manipulation was interfering with migrating $\mathrm{CNC}$ cells, destined to become the craniofacial elements. If this were the case, there would be potential for missing or greatly malformed cartilage structures. Since all elements are present and mostly resemble their control counterparts, we assume that if any damage were done to migrating $\mathrm{CNC}$ cells, it did not reach a threshold where one could rule out the impact of ANR removal. The changes seen in the chondrocranium are thus likely caused by migration interference, and potentially through morphogen downregulation.

\subsection{3: Optic development interrupted with the removal of the forebrain}

Some commonly recurring features seen in $\mathrm{FB}^{-}$specimens are malformed eyes and optic nerves. Some of the observed phenotypes are incomplete lateral eye migration, lack of eye pigment, variable eye shapes, or poorly formed optic nerves. For vertebrate eye development to proceed, an extension of the forebrain from the diencephalic region must interact with the head ectoderm in order to form the optic 
placode and the presumptive neural retina (Lewis, 1904; Gilbert, 2000; Wilson and Houart, 2004). The optic placode then invaginates into the early optic cup, which forms the pigmented and neurosensory layers of the retina (Adler and Canto-Soler, 2007; Eiraku et al., 2011). Thus, the coordinated development of one of the most complex vertebrate sensory structures begins with the forebrain.

During the stages at which we removed the forebrain in $X$. laevis embryos, the optic placode was already present (i.e., NF stages 22 to 28). As such, the surgery did not directly interrupt optic placode formation. However, the placode still requires coordinated signals for the successful continued development of the eye. There are several genes that encode eye-specific transcription factors, including Six 3 and Otx2, but one that emerges as being particularly relevant here is Pax6 (Streit, 2007). Initially expressed in the anterior neural plate during late gastrula stage, the Pax6 transcription factor eventually becomes one of the dominant homeobox genes concentrated in the anterior portion of the vertebrate cortex (Muzio and Mallamaci, 2003; Moreno et al., 2008). Pax6 is necessary for the initial induction of the optic placode, and furthermore, the maintenance of Pax6 expression is required for the subsequent development of the eye (Schlosser and Ahrens, 2004; Kha et al., 2018; Kha et al., 2019). Removing the forebrain can reduce the levels of Pax6 expression in the anterior region of the head, as this is where it is highly concentrated, thus hindering or interrupting completely the developmental trajectory of the eyes.

As in some specimens (Fig. 3.2), we can see that with forebrain removal the eyes are not always present or are incorrectly positioned. The absent eyes could be caused by a disruption in Pax6 expression, leaving the process halted in the placode or early optic cup stage. This could be further investigated with 24-hour post-surgery parasagittal sections paired with an in situ hybridization for Pax6 expression. To 
investigate the late stage changes in eye morphology, we could conduct a histological analysis on $\mathrm{FB}^{-} X$. laevis tadpoles to determine at what point their eye development was arrested. Conducting these tests will also help us conclude that the defects seen in the optic system were caused by reduced Pax6 expression and not physical deformations from the ablation.

\subsection{4: Olfactory development interrupted with the removal of the forebrain}

At NF stage 21, three neurogenic placodes are distinct - the olfactory placode, profundal/trigeminal placode, and dorsolateral placode (Schlosser and Northcutt, 2000). The olfactory placode is of particular interest because it is located at the rostral pole of the developing embryo and it overlays the forebrain, which was the target structure of our surgeries. The olfactory placode, which arises from the lateral neural folds, remains distinct from NF stage 21 to NF stage 30 when the first neurons begin migrating towards the olfactory bulb (Klein and Graziadei, 1983). This means that in the temporal window during which the forebrain was removed, the olfactory placode was a discrete structure and had potential to be ablated along with the forebrain. In removing the olfactory placode before it had the chance to develop into proliferating neurons, I could have disrupted all further functions of the olfactory system including the development of the olfactory epithelium.

The olfactory epithelium is the most commonly affected structure seen in the $\mathrm{FB}^{-}$tadpoles. As discussed above, the nasal organs were affected when cyclopia was induced due to an inability of sensory structures to separate bilaterally. While disruption to the Shh pathway is our main hypothesis for the malformed nasal pits, there is still potential for olfactory placode ablation to be a factor in disrupted development. Since the placode doesn't begin its neuron outgrowth until NF stage 30, 
removing it prior to this would interrupt any further development of the olfactory system, including olfactory ensheathing glia and gonadotropin-releasing hormone neurons that migrate to the pituitary gland (Schwanzel-Fukuda and Pfaff, 1989; Wray et al., 1989). If the olfactory placode is ablated completely then the olfactory epithelium will not be able to develop at all, leaving empty or absent nasal pits. This was not observed in most of the $\mathrm{FB}^{-}$tadpoles; however, one specimen did have a missing right nasal pit (Fig. 3.2F) and another showed little signs of olfactory development (Fig. 3.2H). In these cases, the morphology seen is interpreted to be due to olfactory placode ablation rather than signaling pathway interruption.

This interpretation is supported by observation of specimens with normal optic development but abnormal nasal development (Fig. 3.2D), indicating the cause for this combination may be a lack of neurogenesis due to an absent olfactory placode. During the stages at which surgery was performed, the paired olfactory placodes are flanking the forebrain anteriolaterally so it is possible that in removing the forebrain, and the surface ectoderm, the olfactory placode was removed in the process. However, most specimens we observed that had absent or stunted nasal pits typically also had similar malformations in optic development. While placode ablation is a potential factor in the abnormal development of the olfactory epithelium in some specimens, the co-occurence of these two morphologies suggests that this combined phenotype likely more often arose due to induced cyclopia via interruption of the Shh pathway.

\subsection{5: Bilateral asymmetry}

One characteristic seen both internally and externally on the post-surgery tadpoles is bilateral asymmetry. For example in one specimen (Fig. 3.2F), only one 
normally developed nasal pit was present, the other being absent entirely. This asymmetry was also seen when observing several other cranial structures such as the developing mandible or palatoquadrate. One hypothesis for this result is that the development of left and right sides are occurring simultaneously but are regulated separately. In classical development studies, the left-right body axis was studied closely through surgical manipulations (Blum et al., 2009). Germ layer manipulation experiments were conducted whereby all three germ layers were removed from the mid-dorsal region of neurula embryos of several amphibian species. They rotated the piece of tissue by $180^{\circ}$ before placing it back in the original site and allowing it to heal over. The results showed that the tadpoles had developed complete organ placement inversion, showing that the left and right sides maintained their lateral identity (Spemann, 1906; Pressler, 1911; Meyer, 1913). This bilateral determined fate is also seen in other vertebrates. Lateral manipulation experiments were conducted using chick embryos where presomitic mesoderm from the right half of the thoracic region was transplanted to the cervical region of the recipient. This transplanted tissue maintained not only its thoracic fate but also its bilateral fate, remaining entirely of right-side identity (Kieny et al., 1972). The left side did not mimic that of the right so each axis was entirely independent. This provides some insight into our forebrain removal specimens as they also did not display left-right mimicry.

It is known that the left-right axis is established very early on in development, with some data supporting the idea that this occurs as early as the 4-cell stage (Blum et al., 2009). However, this means that alterations made to one side of the embryo may not impact the other, due to unilateral patterning. Considering this, lateral identity is already well-established by our targeted surgery stages. It is also known that $X$. laevis retains a varying degree of regenerative capabilities throughout different 
stages of development, possibly allowing for post-surgical specimens to recover neural tissue to some extent (Lee et al., 2013; Lee-Liu et al., 2017). During the forebrain removal surgeries, while the goal was to remove equal tissue amounts from both halves, the actual amount of tissue removed may have varied between specimens. If less neural tissue was removed on one side compared to the other, it may have a greater chance at developing more normally in comparison to the other side, leading to unilateral changes to structural elements. This then raises the question - is the severity of structural changes cell population dependent? In Shh inhibition experiments in chicks, the more Shh signaling was inhibited, the more severe the craniofacial malformation was (Marcucio et al., 2005). If more ectodermal cells are left behind, it provides a potential advantage for normal development, allowing for that half to display a normal morphology, whereas the other half would exhibit an abnormal morphology. This can be seen in one specimen (Fig. 3.3C), where the larval pars articularis of the palatoquadrate is articulating normally with Meckel's cartilage on the right side but on the left side it is a nearly unrecognizable structure. This morphology supports the hypothesis that the left and right halves of the developing embryo are organized individually in $X$. laevis, as in other vertebrates (Kieny et al., 1972; Arias et al., 2017).

With respect to cell population dependent effects, an interesting observation noted here was that some specimens displayed a completely normal phenotype following forebrain removal (Fig. 3.2A, J). While every post-surgery specimen that was left to continue development had their forebrain removed, as mentioned above, we cannot confirm that every cell in the forebrain region was removed. We thus hypothesize that there is a threshold of forebrain ectodermal cells that can be left behind in order to display normal development. In other words, if a certain amount of 
ectodermal cells are left behind, then forebrain-specific genes can continue to be expressed at adequate levels, maintaining all signaling pathways that are essential for anterior development. If only one half of the embryo has met this threshold, then only that half will display a less severe or normal morphology. If both halves of the embryo have retained enough ectodermal cells, then both halves will display a less severe or normal morphology. This hypothesis could be investigated by more carefully comparing individual specimens immediately post-surgery (whole mount or histologically) to determine if comparable amounts of tissue were removed. It can be noted that in addition to severity of malformation, degree of recovery is also not attributed to surgeries performed at any one stage.

Variation in the defects observed can also be caused by the difference in surgical precision between specimens. While certain measures were taken to minimize variation in tissue ablation methods, such as screening embryos 24-hours post-surgery for any unwanted morphologies, the tissue is still being removed by hand with a blunt instrument, introducing technical error as a factor that must be considered. As such, much of the hypothesized sources for morphological alterations (i.e. interruption of $S h h$ expression leading to optic cyclopia) require further investigation to rule out technical errors by surgery.

\subsection{6: Forebrain removal does not disrupt the posterior elements of the skull}

Evolutionarily, there is evidence to suggest that the development of the forebrain is related to that of the frontal bone, and the midbrain is to the parietal bone. In one study, the forebrain-midbrain boundary of the three-vesicle stage embryo was found to reside in the same position as the frontal-parietal suture in Reptilia (Fabbri et al., 2017). In anurans, the frontoparietal bone has been an interesting point of study as 
it does not develop from two ossification centers, which would suggest an ossification center for the frontal and another for the parietal, followed by their fusion into one structure (Roček, 1988). Instead, it develops from one ossification center and is entirely CNC-derived, the latter point being inconsistent with the frontal-parietal fatemap seen in the salamander Ambystoma mexicanum, more commonly known as the axolotl (Piekarski et al., 2014). If the frontoparietal of anurans follows the same forebrain-midbrain developmental relationship hypothesized by Fabbri et al. (2017), then we would expect to see a disruption to the development of the frontal portion of the frontoparietal when the forebrain is removed, leaving the parietal portion undisturbed. This is indeed what was observed in $\mathrm{FB}^{-}$tadpoles.

The frontoparietal fontanelle extends from the middle of the subocular fenestra to the middle of the otic capsule (Fig. 2.2D-F; see also Trueb and Hanken, 1992). While we cannot say with great precision at this time where the boundary between the frontal and parietal portions of the frontoparietal bone would reside, the middle of fontanelle occurs just posterior to the ascending process of the palatoquadrate. Interestingly, it is at this point and anterior that we see morphological changes in our post-surgery specimens. For example, I observed an abnormally shaped anterior margin of the fontanelle, suggesting the anterior, frontal portion of the frontoparietal would also have been abnormally shaped had the specimen lived to ossification. Thus, the frontal bone may indeed rely on information from the developing forebrain to achieve a normal morphology.

On the other hand, in all the specimens, structures posterior to this point - i.e., the parachordal cartilage, the occipital arch, the ceratobranchial elements, and the otic capsules - remained completely unaffected (Fig. 3.2). Even in the most severely malformed specimens (Fig. 3.2K), where the entire head had been shortened and the 
tail was no longer straight, the otic capsules and posterior ceratobranchial elements remained normal. It should be noted that it is difficult to say whether the slight alterations in some non-craniofacial elements, such as the anterior ceratobranchial elements, are due to forebrain removal or simply that the structures around it (i.e., palatoquadrate) are greatly affected and subsequently have spatial influence on their development. It can also be noted that the development of individual elements may have been disrupted, but their positioning relative to each other has remained mostly unchanged. This is similar to results seen in Shh inhibition experiments in chick where the skeletal elements display physical changes but the nature of their articulations are unchanged (Marcucio et al., 2005).

Combined with data from previous studies, our data supports the hypothesis of a developmental relationship between the forebrain and midbrain with the frontal and parietals portions of the frontoparietal, respectively, regardless of whether the two elements are united as in the case of the anuran frontoparietal. Our observations also suggest that further than just the frontoparietal, the forebrain-midbrain boundary may play a larger role in anterior-posterior patterning of the skull as a whole, with the forebrain governing development of anterior elements (i.e., palatal, rostral, maxillary, and mandibular structures) and the midbrain relating to the development of the posterior elements (i.e., otic capsules, occipital arch, posterior hyoid arch). The regions on either side of this border appear to develop somewhat independently from each other using spatial information from the regions of the central nervous system developing adjacently. Continued studies on the forebrain-midbrain boundary and its part in patterning the cranial elements can help elucidate the direct role of the forebrain in guiding anterior skull development in tetrapods. 


\subsection{7: Pigmentation}

Our $\mathrm{FB}^{-}$specimens display very irregular melanocyte patterns. Some specimens showed a severe reduction in melanocytes, while others displayed uneven bilateral pigmentation (Fig. 3.2F). Melanocytes are derived from cranial neural crest cells early in development and migrate to various parts of the body (Gilbert, 2000; Silver et al., 2006). While the degree of pigmentation (i.e., the amount of melanin) varies slightly among normal $X$. laevis tadpoles, the overall patterns or pigmentation and the shapes of individual melanocytes typically remain the same. The observed reduction in melanocytes present in the head could be another result of decreased Fgf2/8 expression, as knockdown experiments of these genes have been shown to impede melanocyte development (Abzhanov and Tabin, 2004). The observed bilateral asymmetry may arise the same way as the asymmetry we saw in sensory and cartilage structure development, i.e., that left pigmentation processes are independent of the right. We also observed that some melanocytes congregated and produced melanin as a shaded area rather than the normal individual specks seen on the dorsal surface. Although we are likely disrupting the ability for melanocytes to migrate to their target destination, we may also be disrupting the internal system of melanin production and distribution. Future experiments would be needed to distinguish between these alternatives.

\subsection{8: Removal of forebrain reduced viability of experimental specimens}

One setback for our results was the inability of our experimental animals to reach ossification stages, and thus prevented the direct observation of the consequences of forebrain removal on skull bone development and resulting morphology. While we can make inferences on how the removal of the forebrain 
could affect bone formation through studying the disrupted development of the cartilaginous structures (see Chapter 4), direct observation of the bony skull in $\mathrm{FB}^{-}$ specimens would have been ideal for revealing the role the brain plays in shaping the skull.

There are two potential reasons why the tadpoles did not survive. The first could be an interrupted feeding mechanism. Since one of the main structures affected in the chondrocranium was the mandible, as well as the ceratohyals, the tadpoles may have lacked the ability to feed properly. Since X. laevis tadpoles feed via suction, disruption to these elements can have a detrimental effect on this complex feeding system. The tadpoles may not have been able to obtain nutrients necessary for survival, thus leading to their early death. Next, tadpole survivability could be attributed to the importance of the forebrain for key developmental processes. The forebrain likely plays a critical role in conducting numerous developmental events; therefore, the lack of a fully functioning forebrain could be causing many downstream effects that led to the early death of tadpoles. In other brain removal experiments, the tadpoles did not develop past NF stage 48 , suggesting that there may be a maximum age that the tadpoles are capable of developing to in the absence of certain brain tissues (Herrera-Rincon et al., 2017). When nosip, a critical factor in forebrain neurogenesis and neural stem-cell renewal, was knocked down in X. laevis, it resulted in holoprosencephaly and the oldest specimen only reached NF stage 42 (Hoffmeister et al., 2017). Considering that many neural manipulation experiments do not produce fully viable animals, it is unlikely that the $\mathrm{FB}^{-}$tadpoles would survive to full ossification. 


\section{Chapter 4: Discussion and Conclusions}

The vertebrate skull has undergone many modifications throughout its evolutionary history, and although these changes reflect the effects of several contributing factors, one factor that remains incompletely understood is the role the brain has played in groups of vertebrates outside of certain well-studied groups, such as primates and birds. Encephalization has influenced overall skull shape in these groups through modifications of individual bones, primarily the frontal and parietal bones (Morriss-Kay, 2001; Jiang et al., 2002; Maddin et al., 2016); however, other elements are also impacted - i.e., craniofacial elements such as the nasal, maxillary, and mandibular structures - but remain less well understood in terms of their response to changes in the brain. To understand the role the forebrain may have played in the evolution of the skull, it must be studied from physical, genetic, and biochemical perspectives. Here, I focused on the physical changes incurred following forebrain removal and discuss the biochemical implications on early developmental signaling.

To further our understanding of the brain-bone relationships in evolution, it is important to establish which developmental trends are seen across vertebrate species. Amphibians remain the most understudied group of vertebrates with respect to investigating how the brain interacts with and shapes the skull. In this thesis, I attempted to remedy this issue by first generating a detailed description of normal skull development in the model species Xenopus laevis (Chapter 2) and then by performing forebrain removal experiments to observe the consequences on skull development (Chapter 3). I then interpret the results as they relate to potential biochemical causes, such as which signaling pathways are disrupted when 
the forebrain is removed. Here, I summarize the findings of this research and discuss how they contribute to the complex topic of vertebrate skull evolution.

\section{1: Changes to the chondrocranium via forebrain removal can interfere with chondro-dermatocranial interactions}

As vertebrates evolved and skull elements were modified, one must consider the relationship between the bone and the neighbouring soft tissue to understand the mechanistic bases of such modifications. Here, the goal was to explore the role of one such soft tissue structure - the brain - and to gain the first insights into the nature of its influence on shaping the skull in an amphibian species. Even though the postsurgery specimens of $X$. laevis were not viable to ossification stages, I first demonstrated the brain does impact skull development and then used the integrated development of the chondrocranium with the dermatocranium to make inferences on the brain-bone relationship.

Following forebrain removal, the cartilaginous structures affected can be grouped into four main categories: palatal and rostral, mandibular, hyoid arch, and subocular developmental interference. In the palatal and rostral region, it was the suprarostral plate and anterior palatoquadrate that were the most impacted structures, resulting in malformed nasal foramina and poor articulation with Meckel's cartilage (Fig. 3.3). Malformations of the mandibular region included bilateral asymmetry between the two halves of Meckel's cartilage and changes to the infrarostral, including increased curvature or complete inversion (Fig. 3.4). The ceratohyals were the only element of the hyoid arch that showed alteration due to forebrain removal; however, it did influence nearby structures such as the basihyobranchial (Fig. 3.5). Finally, the subocular region has malformations in several structures including the subocular cartilage, the ventrolateral process of the palatoquadrate, and the ascending 
process of the palatoquadrate, resulting in misshapen subocular foramina (Fig. 3.6). Additionally, there were some changes to the anterior region of the frontoparietal fontanelle (Fig. 3.3).

As discussed in Chapter 2, we can use the chondrocranium to study the dermatocranium as the cartilage acts as a primary scaffolding to guide dermal bone ossification (Kawasaki and Richtesmeier, 2017; Pitirri et al., 2020). When the forebrain was removed in $X$. laevis embryos, a series of physical changes on the tadpole chondrocranium were induced. Since the dermatocranium uses cartilaginous structures as scaffolding during development, I can hypothesize that the interfering with forebrain development can have a direct impact on the dermatocranium in $X$. laevis. Examples of chondrocranial structures that were inferred here that play a critical role in the development of their bony counterpart include the frontoparietal fontanelle and the frontoparietal bone, and the mandibular cartilages and the mandibular bones (Trueb and Hanken, 1992; Kawasaki and Richtsmeier, 2017). Since the fontanelle acts as a guide for the initial ossification centres of the frontoparietal bone (Trueb and Hanken, 1992; Senevirathne et al., 2017), an abnormally shaped fontanelle will likely cause the frontoparietal bone to be misshapen as well. For example, in one specimen (Fig. 3.3D), we saw a narrowing of the anterior region of the frontoparietal fontanelle. It is likely the frontoparietal would therefore be more narrow anteriorly as well. When observing changes in Meckel's cartilage induced by forebrain removal, we can theorize what the resulting mandibular bones might look like. We may see asymmetrical dentary bones and angulosplenials as well as a much thicker angulosplenial due to the thickening of Meckel's cartilage observed in some specimens. The abnormally shaped infrarostral may cause the paired dentary bones to poorly articulate along the midline if they articulate at all. This would greatly impact 
the tadpole's ability to feed and survive, as discussed in Chapter 3 with regards to viability.

My findings support the hypothesis that removal of the forebrain can have structural implications on the ossification of the dermatocranium, as many of the resulting chondrocranial changes were fairly substantial in degree. In removing the forebrain, we are disrupting pathways required for the normal development of key cartilaginous structures, and in doing so, we are modifying the structural template needed to establish many important bony elements of the head.

\section{2: The forebrain is required for normal optic and olfactory sensory development}

Two of the most consistent outcomes observed when noting features of $\mathrm{FB}^{-}$ tadpoles were olfactory and optic cyclopia, often with both occurring simultaneously. Two $\mathrm{FB}^{-}$tadpoles displayed severe optic cyclopia and many others displayed paired, but mildly to moderately, malformed eyes. Features of these tadpoles included poorly developed optic nerves and disintegrating pigmented retinal epithelium (Fig. 3.2F-I). Severe olfactory cyclopia was seen in four $\mathrm{FB}^{-}$tadpoles whereas paired, but malformed, nasal pits were more commonly observed (Fig. 3.2). Features of these tadpoles included fused nasal pits containing paired olfactory epithelia and small or uneven nasal pits. The co-occurring nature of these resulting features suggests a developmental connection between them, and similarities with features observed in holoprosencephalic mice implicate a potentially shared biochemical cause (Heavner and Pevny, 2012).

The olfactory and optic systems develop from the olfactory and optic placode, respectively. The optic vesicles were formed before the forebrain was removed, as the diencephalic protrusion had already interacted with surface ectoderm to form the optic 
placode. The optic vesicles were avoided during forebrain removal and were not physically ablated. Conversely, the olfactory placodes did pose a risk of being ablated during forebrain removal as they overlay and flank the forebrain during our targeted surgery stages (NF stage 22-28) (Schlosser and Northcutt, 2000). In the specimens where olfactory development was completely inhibited, the cause can likely be attributed to olfactory placode ablation since removal of this structure will lead to its failure to develop (Akutsu et al., 1992). In the specimens where both olfactory and optic development are altered, these morphologies are likely correlated with disruptions to signaling patterns resulting from forebrain removal, as both can be linked to decreased levels of Shh and Fgf8 signalling.

When removing forebrain tissue in the $X$. laevis embryos, we also removed the overlying surface ectoderm, which will become dorsal skin, not essential for structural development (Vieira et al., 2010). We also removed the neuroepithelium overlying the anterior most region of the forebrain, which is the ANR (Abzhanov and Tabin, 2004). Since the function and development of the ANR and forebrain are so closely related, these were removed together. In addition to the surface ectoderm and the neuroepithelium, there are other nearby tissues that are potentially disrupted during forebrain removal surgery. One of these is migrating cranial neural crest (CNC), specifically the mandibular stream neural crest, as opposed to the hyoid and branchial streams (Hanken and Gross, 2005; Gross and Hanken, 2008). If the mandibular stream of $\mathrm{CNC}$ was severely disrupted as a byproduct of forebrain removal, then there is a possibility that major structural defects could occur, such as abnormal development of the structures it gives rise to but primarily, one would expect those structures to be missing entirely. In several other neural crest manipulation experiments, removal of the cranial neural crest caused certain structures to fail to 
form, such as the thymus gland (Bockman and Kirby, 1984) or the structures would take on the fate of the grafted tissue (Noden, 1983). If during our forebrain removal surgeries we removed mandibular $\mathrm{CNC}$ or the olfactory placodes, then we'd expect the resulting tadpoles to be missing chondrocranial elements and olfactory organs. This was not the case in our experimental tadpoles; we observe that all the normal structures were present but some were malformed. I therefore interpret this to indicate the source of these developmental anomalies was likely not by disruption of $\mathrm{CNC}$ or sensory placodes but instead by the lack of forebrain tissue and adequate levels of the signals it releases. This supports the contention that it is the forebrain impacting the development of chondrocranial structures and not disruption of nearby tissues.

It is important to establish the difference between the role that the forebrain plays in sensory development rather than the placodal role. The placodes typically give rise to their designated structures (i.e., the olfactory bulb and olfactory epithelium for the olfactory placode, and the lens and retinal pigmented epithelium for the optic placode) whereas the forebrain and surrounding neuroepithelium acts as a signal origin for $F g f 8$ and $S h h$, which ensure the subsequent development of said structures (Abzhanov and Tabin, 2004; Vieira et al., 2010). This is demonstrated in our $\mathrm{FB}^{-}$tadpoles because the sensory structures in question are still present, with a few exceptions; however, their development has been sufficiently interrupted. These are notable results because much of forebrain development is studied in mouse and chick, less so in amphibians (Morriss-Kay, 2001; Hu and Marcucio, 2009). Since we can observe a similar morphology in a model anuran when the forebrain is removed, as we can with mouse and chick, we can further our understanding of vertebrate neural development of all species that phylogenetically come after anurans. 
The morphologies seen in this thesis can also be related to similar craniofacial disorders seen in humans. By removing the forebrain of $X$. laevis embryos, we induced craniofacial deformities as well as sensory organ disruption. Craniofacial defects caused by a malformed forebrain are also observable in humans and are regularly modeled in mouse and chick (Fitz, 1994; Bolande, 1997; Cohen, 2006; Raam et al., 2010; Vega-Lopez et al., 2018). By removing the forebrain, I induced cyclopic-like characteristics, which resemble those of human congenital cyclopia (Chiang et al., 1996). I also caused palatal malformations, which are also observed in several human craniofacial disorders (Dubey and Saint-Jeannet, 2017).

Since I observed that interrupting forebrain development in X. laevis induced several craniofacial anomalies, my results support the notion that amphibians are a good model for human craniofacial and sensory pathologies. Not only do they show similar morphologies when manipulating the forebrain, suggesting similar biochemical pathways are at play, but they also produce significantly more offspring than mouse and chicken in a shorter period of time, are much easier to manipulate during early development, and possess many genes that are homologous with humans (Tandon et al., 2017). Further study of fundamental aspects of amphibian skull development may thus contribute to clarifying outstanding questions of human pathology in a more amenable system.

\section{3: Development of the chondrocranium is anteroposteriorly regionalized by instructions from the brain}

A developmental relationship between the frontal and parietal bones and the fore- and midbrain, respectively, has been discussed in reptilians and birds (Fabbri et al., 2017). Whether such regionalization exists in amphibians has not been previously explored. Investigating this in anurans is a challenge, since, as previously mentioned, 
the frontal and parietal are indistinguishably fused in anurans. The frontoparietal evolved exclusively in anurans as early as the Jurassic, with signs of near fusion between the frontal and parietal bones dating to the Triassic (Roček, 1988). Therefore, no living anuran, not even the most primitive members, can shed light on the existence of such a relationship. Interestingly, I found that any structural modifications that were made to the frontoparietal fontanelle as a result of removing the forebrain were only seen in the anterior region. This would be the region of the frontoparietal that corresponds to the frontal bone of other tetrapods. Therefore, in removing the forebrain, I appear to have been only interfering with the frontal portion of the frontoparietal. This finding suggests that anurans are similar to the amniotes studied thus far in that forebrain development discretely correlates with frontal bone development.

In addition to the anteroposterior frontoparietal fontanelle division, there is also an anteroposterior division of the chondrocranium as a whole. In reviewing the modifications made to the chondrocranium as a result of forebrain manipulation, only structures anterior to the otic capsules were affected, not including the ceratobranchial elements. If a suture did exist within the anuran frontoparietal, it has been hypothesized to reside near the mid-length of the bone. This is thought to be the case because in other amphibians with a separate frontal and parietal, the two bones are equal in length (Romer and Parsons, 1977). This hypothesized frontal-parietal border in anurans lines up with the region between the otic capsules and the ascending process of the palatoquadrate.

The forebrain-midbrain boundary that typically correlates with the frontoparietal suture may have further influence than just the frontal and parietal bones (Fabbri et al., 2017). If we observe that only anterior structures are affected 
after forebrain removal, then we can infer that the forebrain plays an important role in the development of the anterior region of the head, extending beyond the forebrainfrontal bone relationship. This may be due to how these cranial structures respond to signals from the forebrain but this is difficult to discuss as most $X$. laevis signaling center research focuses on the blastula stage rather than the three-vesicle stage of the developing brain (Kuroda et al., 2004). If signaling in the $X$. laevis three-vesicle stage brain is similar to that in chick and mouse, then we can make a few assumptions about the role the forebrain plays in anterior cranial patterning. For example, the midbrain has a higher concentration of Wnt signalling (Millet et al., 1999) compared to the concentrated $F g f 8$ expression from the anterior neural ridge (ANR) (Eagleson et al., 1995; Vieira et al., 2010). The variation in signals released from the forebrain and midbrain regions could be not only impacting the frontal and parietal bones, but also the anterior and posterior cranial elements of the head, respectively. These expression domains also may line up with $\mathrm{CNC} /$ mesoderm boundaries. The cartilaginous structures affected in our $\mathrm{FB}^{-}$tadpoles seem to be associated with neural crest derived bones in mouse models (D'Souza et al., 2010). We also see congruency when reviewing the lack of disruption in otic capsule development, with the otic capsule being mesodermally derived (Thompson et al., 2012).

\section{4: Future Directions}

In this thesis, I removed the forebrain in $X$. laevis embryos to study the physical changes, if any, that were incurred on the skull. Based on the results of previous research performed on amniote model species and the similarities seen in my experimental animals, I formulated several hypotheses about the biochemical causes of my results: 1) forebrain removal leads to reduced Shh expression, 2) forebrain removal leads to reduced $F g f 8$ expression via interference with Shh signalling, 3) 
forebrain removal leads to reduced Pax6 expression as it is expressed in the anterior neural region and 4) combined, these factors influence the development of the skull in $X$. laevis. To test these hypotheses, one could conduct in situ hybridization protocols or immunohistochemistry for these three genes/proteins in question. This would allow us to visualize their gene expression to confirm whether removal of the forebrain is indeed affecting their expression, and thus interfering with several downstream functions. While immunohistochemistry and in situ hybridization cannot give us information about the expression levels, it can tell us if expression remains in that region. If our hypotheses are correct, then removing the forebrain would reduce spatiotemporal expression of each gene mentioned, and further implicate their role in olfactory, optic, and chondrocranial structural development.

To corroborate our results, knockdown experiments could also be conducted to study whether the same morphologies arise. However, several of the genes discussed here have multiple functions (e.g., Shh and limb patterning; Yang and Niswander, 1995) so knockdown may have unwanted effects in other domains, making it difficult to conclude the proper source of the malformations. To combat this, more targeted knockdown approaches must be conducted. Such targeted approaches have been conducted in chick, specifically with Shh interruption to study forebrain-facial interactions, by injecting hybridoma cells expressing anti-Shh antibodies (Marcucio et al., 2005). To conduct an experiment more targeted, forebrain-regulatory genes must be knocked down. A prime candidate is Nosip - a gene that has recently been found to be critical for normal forebrain development and when deleted in mouse and $X$. laevis causes holoprosencephaly and reduction in brain size, respectively (Hoffmeister et al., 2017). It has previously been studied with respect to its function in neural development, but it could very well be examined for 
chondrocranial defects, as the $X$. laevis specimens in Hoffmeister et al. (2017) lived at least until NF stage 42. Therefore, it is possible that Nosip knockdown tadpoles would be capable of living until ossification stages, which would be beneficial in confirming the impact of forebrain interference on dermatocranium ossification patterns.

\section{5: Conclusions}

The goal of this MSc thesis was to further our understanding of the role that the forebrain plays in cranial development of tetrapods by exploring brain-skull interactions in the amphibian species Xenopus laevis. Here, I have shown that the forebrain is necessary for normal skull development, and additionally for normal sensory structure development. Removal of the forebrain impacts structural elements of the chondrocranium in the sense that while all required elements are present, they display morphological changes from mild to severe developmental interruptions. Secondly, we have shown that removal of the forebrain severely interferes with sensory development of olfactory and optic structures in the form of induced cyclopia. This research is essential in furthering our understanding of how the brain has shaped cranial evolution, as well as sensory development, across vertebrate species by filling a critical gap in knowledge, i.e., the role of the brain in the development of the skull in an amphibian species. This embryological approach to tetrapod evolution can continue to provide vital insight into studying and interpreting evolutionary patterns in both extinct and extant species. 


\section{$\underline{\text { Appendices }}$}

Appendix A: Xenopus laevis staging series specimen numbers and sequence of ossification

Table A1: Number of Xenopus laevis specimens included in the staging series, cartilage and bone stain

\begin{tabular}{|l|l|l|l|}
\hline $\begin{array}{l}\text { NF } \\
\text { Stage }\end{array}$ & Total number of specimens & $\begin{array}{l}\text { NF } \\
\text { Stage }\end{array}$ & Total number of specimens \\
\hline $\mathbf{4 1}$ & 2 & $\mathbf{5 4}$ & 8 \\
\hline $\mathbf{4 2}$ & 2 & $\mathbf{5 5}$ & 11 \\
\hline $\mathbf{4 3}$ & 3 & $\mathbf{5 6}$ & 9 \\
\hline $\mathbf{4 4}$ & 4 & $\mathbf{5 7}$ & 8 \\
\hline $\mathbf{4 5}$ & 6 & $\mathbf{5 8}$ & 9 \\
\hline $\mathbf{4 6}$ & 4 & $\mathbf{5 9}$ & 3 \\
\hline $\mathbf{4 7}$ & 7 & $\mathbf{6 0}$ & 2 \\
\hline $\mathbf{4 8}$ & 3 & $\mathbf{6 1}$ & 2 \\
\hline $\mathbf{4 9}$ & 8 & $\mathbf{6 2}$ & 4 \\
\hline $\mathbf{5 0}$ & 2 & $\mathbf{6 3}$ & 3 \\
\hline $\mathbf{5 1}$ & 5 & $\mathbf{6 4}$ & 3 \\
\hline $\mathbf{5 2}$ & 2 & $\mathbf{6 5}$ & 4 \\
\hline $\mathbf{5 3}$ & 7 & 4 & \\
\hline
\end{tabular}


Table A2: Cranial ossification sequence in Xenopus laevis

Table 1. Cranial ossification sequence in Xenopus laevis

\begin{tabular}{|c|c|c|c|c|c|}
\hline Stage & This Study & $\begin{array}{l}\text { Trueb \& Hanken } \\
\text { (1992) }\end{array}$ & $\begin{array}{l}\text { Bernasconi } \\
\quad(1951)\end{array}$ & $\begin{array}{l}\text { Sedra \& Michael } \\
\text { (1957) }\end{array}$ & $\begin{array}{l}\text { Brown } \\
(1980)\end{array}$ \\
\hline 54 & $\begin{array}{l}\text { Parasphenoid (-56) } \\
\text { Frontoparietal }(-57)\end{array}$ & Frontoparietal & Frontoparietal & - & - \\
\hline 55 & & $\begin{array}{l}\text { Parasphenoid } \\
\text { Exoccipital }(-56) \\
\text { Prootic }(-57)\end{array}$ & $\begin{array}{l}\text { Parasphenoid } \\
\text { Exoccipital } \\
\text { m. Angulosplenial } \\
\text { Maxilla } \\
\text { Teeth } \\
\text { Premaxilla }\end{array}$ & $\begin{array}{l}\text { Frontoparietal } \\
\text { Parasphenoid }\end{array}$ & $\begin{array}{l}\text { Frontoparietal } \\
\text { Parasphenoid } \\
\text { Exoccipital } \\
\text { Prootic } \\
\text { m. Angulosplenial }\end{array}$ \\
\hline 56 & $\begin{array}{l}\text { ant. Vertebrae } \\
\text { m. Angulosplenial }(-57) \\
\text { Otic capsule }(-58)\end{array}$ & m. Angulosplenial & Prootic & - & - \\
\hline 57 & $\begin{array}{l}\text { Prootic } \\
\text { Exoccipitals }\end{array}$ & Maxilla & - & $\longrightarrow$ & $\begin{array}{l}\text { Maxilla } \\
\text { Premaxilla } \\
\text { Teeth }\end{array}$ \\
\hline 58 & $\begin{array}{l}\text { Maxilla }(-62) \\
\text { Epiotic }(-59)\end{array}$ & $\begin{array}{l}\text { Premaxilla }(-59) \\
\text { Nasal }(-60)\end{array}$ & Nasal & $\begin{array}{l}\text { Prootic } \\
\text { Exoccipital } \\
\text { Maxilla } \\
\text { Nasal }\end{array}$ & - \\
\hline 59 & & - & Dentary & $\begin{array}{l}\text { Pars interna plectri } \\
\text { Operculum }\end{array}$ & Nasal \\
\hline 60 & $\begin{array}{l}\text { Premaxilla }(-62) \\
\text { Nasals }(-63) \\
\text { Maxillary Teeth }(-62)\end{array}$ & $\begin{array}{l}\text { Septomaxilla } \\
\text { Teeth } \\
\text { I. Angulosplenial (-61) } \\
\text { Dentary (-62) }\end{array}$ & $\begin{array}{l}\text { I. Angulosplenial } \\
\text { Septomaxilla } \\
\text { Squamosal } \\
\text { Pterygoid }\end{array}$ & $\begin{array}{l}\text { Septomaxilla } \\
\text { Premaxilla } \\
\text { Teeth } \\
\text { Angulosplenial } \\
\text { Dentary }\end{array}$ & - \\
\hline 61 & $\begin{array}{l}\text { Dentary (-63) } \\
\text { I. Angulosplenial (-62) }\end{array}$ & $\begin{array}{l}\text { Pterygoid }(-62) \\
\text { Tympanic annulus }(-62) \\
\text { Pars externa plectri }(-62)\end{array}$ & $\begin{array}{l}\text { Collumella } \\
\text { ) }\end{array}$ & $\begin{array}{l}\text { Pars media plectri } \\
\text { Pars externa plectri } \\
\text { Tympanic Annulus }\end{array}$ & - \\
\hline 62 & Septomaxilla (-63) & $\begin{array}{l}\text { Pars media plectri }(-63) \\
\text { Squamosal }(-63)\end{array}$ & Vomer & - & $\longrightarrow$ \\
\hline 63 & $\begin{array}{l}\text { Sphenethmoid } \\
\text { Squamosal }\end{array}$ & Vomer $(-64)$ & - & Pterygoid & - \\
\hline 64 & Pterygoid & Sphenethmoid (-66) & 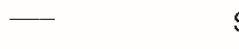 & Squamosal & - \\
\hline 65 & $\begin{array}{l}\text { Vomer } \\
\text { Columella }\end{array}$ & Pars articularis & - & & - \\
\hline 66 & - & - & - & Sphenethmoid & \\
\hline $66+$ & - & Operculum & $\begin{array}{l}\text { Sphenethmoid } \\
\text { Pars articularis } \\
\text { Alary cartilage } \\
\text { Planum antorbital }\end{array}$ & $\overline{l e}$ & - \\
\hline
\end{tabular}


Appendix B: Xenopus laevis $\mathrm{FB}^{-}$specimen numbers, stage, and methods schematic

Table B1: Xenopus laevis $\mathrm{FB}^{-}$specimen numbers and stage of surgery

\begin{tabular}{|l|l|}
\hline $\begin{array}{l}\text { NF Stage at point of } \\
\text { surgery }\end{array}$ & Total number of specimens $(\mathbf{n}=\mathbf{3 5})$ \\
\hline $\mathbf{2 2}$ & 2 \\
\hline 23 & 4 \\
\hline 24 & 7 \\
\hline 25 & 5 \\
\hline 26 & 4 \\
\hline 27 & 5 \\
\hline 28 & 8 \\
\hline
\end{tabular}

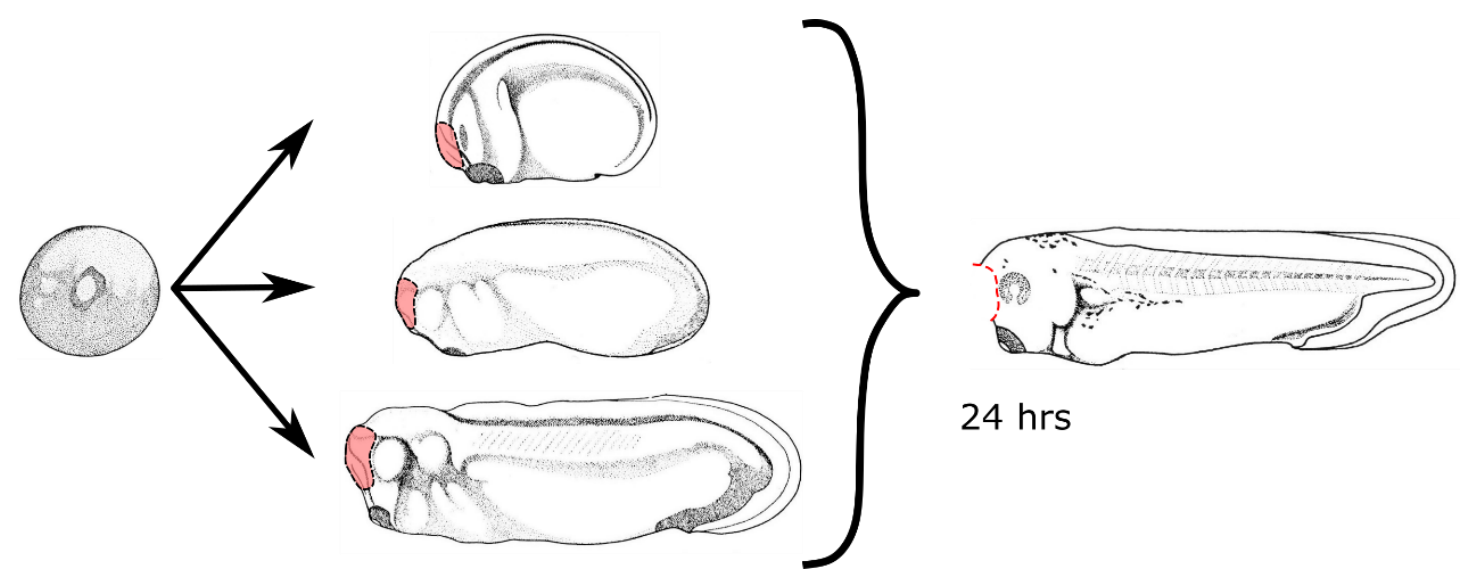

Figure B1: Xenopus laevis forebrain removal surgery schematic. Forebrain removal conducted on $X$. laevis embryos from NF stage 22 to 28. Forebrain tissue, and overlying dorsal skin, was removed with a tungsten needle ( $1 \mu \mathrm{m}$ thickness), avoiding the optic vesicle. 24 hours later, recovered embryos showed an indentation in forebrain 


\section{$\underline{\text { References }}$}

Abzhanov, A., \& Tabin, C. J. (2004). Shh and Fgf8 act synergistically to drive cartilage outgrowth during cranial development. Developmental Biology, 273(1), 134-148. https://doi.org/10.1016/j.ydbio.2004.05.028

Adler, R., \& Canto-Soler, M. V. (2007, May 1). Molecular mechanisms of optic vesicle development: Complexities, ambiguities and controversies.

Developmental Biology. Academic Press Inc. https://doi.org/10.1016/j.ydbio.2007.01.045

Akutsu, S., Takada, M., Ohki-Hamazaki, H., Murakami, S., \& Arai, Y. (1992). Origin of luteinizing hormone-releasing hormone (LHRH) neurons in the chick embryo: Effect of the olfactory placode ablation. Neuroscience Letters, 142(2), 241-244.

Anderson, D. J. (1997). Cellular and molecular biology of neural crest cell lineage determination. Trends in Genetics, 13(7), 276-280.

Arias, C. F., Herrero, M. A., Stern, C. D., \& Bertocchini, F. (2017). A molecular mechanism of symmetry breaking in the early chick embryo. Scientific Reports 2017 7:1, 7(1), 1-6. https://doi.org/10.1038/s41598-017-15883-8

Bachiller, D., Klingensmith, J., Kemp, C., Belo, J. A., Anderson, R. M., May, S. R., ... De Robertis, E. M. (2000). The organizer factors Chordin and Noggin are required for mouse forebrain development. Nature, 403(6770), 658-661. https://doi.org/10.1038/35001072

Balanoff, A. M., Bever, G. S., Colbert, M. W., Clarke, J. A., Field, D. J., Gignac, P. M., ... Witmer, L. M. (2016). Best practices for digitally constructing endocranial casts: examples from birds and their dinosaurian relatives. Journal of Anatomy, 229(2), 173-190. https://doi.org/10.1111/joa.12378

Bernasconi, A. F. (1951). Über den Ossifikation Modus bei Xenopus laevis Daud. Mem. Soc. Helvet. Sci. Nat., 79, 191-252.

Blackburn, D. C., Paluh, D. J., Krone, I., Roberts, E. M., Stanley, E. L., \& Stevens, N. J. (2019). The Earliest Fossil of the African Clawed Frog (Genus Xenopus) from Sub-Saharan Africa. Journal of Herpetology, 53(2), 125. https://doi.org/10.1670/18-139

Blum, M., Beyer, T., Weber, T., Vick, P., Andre, P., Bitzer, E., \& Schweickert, A. (2009, June 1). Xenopus, an ideal model system to study vertebrate left-right asymmetry. Developmental Dynamics. John Wiley \& Sons, Ltd. https://doi.org/10.1002/dvdy.21855

Bockman, D. E., \& Kirby, M. L. (1984). Dependence of thymus development on derivatives of the neural crest. Science, 223(4635), 498-500. https://doi.org/10.1126/SCIENCE.6606851 
Bolande, R. P. (1997). Neurocristopathy: Its Growth and Development in 20 Years. Pediatric Pathology \& Laboratory Medicine, 17(1), 1-25.

https://doi.org/10.1080/15513819709168343

Bonfanti, P., Saibene, M., Bacchetta, R., Mantecca, P., \& Colombo, A. (2018). A glyphosate micro-emulsion formulation displays teratogenicity in Xenopus laevis. Aquatic Toxicology, 195, 103-113. https://doi.org/10.1016/j.aquatox.2017.12.007

Brown, S. M. (1980). Comparative ossification in tadpoles of the Genus Xenopus (Anura, Pipidae). Master's thesis. San Diego, CA: San Diego State University.

Cervantes-Diaz, F., Contreras, P., \& Marcellini, S. (2017). Evolutionary origin of endochondral ossification: the transdifferentiation hypothesis. Development Genes and Evolution, 227(2), 121-127. https://doi.org/10.1007/s00427-0160567-y

Chen, J., Jacox, L. A., Saldanha, F., \& Sive, H. (2017). Mouth development. Wiley Interdisciplinary Reviews: Developmental Biology, 6(5). https://doi.org/10.1002/wdev.275

Chiang, C., Litingtung, Y., Lee, E., Young, K. E., Corden, J. L., Westphal, H., \& Beachy, P. A. (1996). Cyclopia and defective axial patterning in mice lacking Sonic hedgehog gene function. Nature, 383(6599), 407-413. https://doi.org/10.1038/383407a0

Clack, J. A. (2002). Patterns and processes in the early evolution of the tetrapod ear. Journal of Neurobiology, 53(2), 251-264. https://doi.org/10.1002/NEU.10129

Cohen, M. M. (2006). Holoprosencephaly: Clinical, anatomic, and molecular dimensions. Birth Defects Research Part A: Clinical and Molecular Teratology, 76(9), 658-673. https://doi.org/10.1002/bdra.20295

Cordero, D., Marcucio, R., Hu, D., Gaffield, W., Tapadia, M., \& Helms, J. A. (2004). Temporal perturbations in sonic hedgehog signaling elicit the spectrum of holoprosencephaly phenotypes. Journal of Clinical Investigation, 114(4), 485494. https://doi.org/10.1172/jci19596

Cordero, D. R., Brugmann, S., Chu, Y., Bajpai, R., Jame, M., \& Helms, J. A. (2011). Cranial neural crest cells on the move: Their roles in craniofacial development. American Journal of Medical Genetics, Part A, 155(2), 270-279. https://doi.org/10.1002/ajmg.a.33702

Couly, G., \& Le Douarin, N. M. (1988). The fate map of the cephalic neural primordium at the presomitic to the 3 -somite stage in the avian embryo. Development, 103, 101-113.

Creuzet, S., Schuler, B., Couly, G., \& Le Douarin, N. M. (2004). Reciprocal 
relationships between Fgf8 and neural crest cells in facial and forebrain development. Proceedings of the National Academy of Sciences of the United States of America, 101(14), 4843-4847.

https://doi.org/10.1073/pnas.0400869101

D’Souza, R. N., Ruest, L.-B., Hinton, R. J., \& Svoboda, K. K. H. (2010). Development of the Craniofacial Complex. In Bone and Development. Topics in Bone Biology (Vol. 6, pp. 153-181). Springer, London. https://doi.org/10.1007/978-1-84882-822-3_10

De Sá, R. O., \& Swart, C. C. (1999). Development of the suprarostral plate of Pipoid Frogs. Journal of Morphology (1931), 240(2), 143-153.

Dubey, A., \& Saint-Jeannet, J. P. (2017, March 1). Modeling Human Craniofacial Disorders in Xenopus. Current Pathobiology Reports. Springer. https://doi.org/10.1007/s40139-017-0128-8

Dufton, M., Hall, B. K., \& Franz-Odendaal, T. A. (2012). Early Lens Ablation Causes Dramatic Long-Term Effects on the Shape of Bones in the Craniofacial Skeleton of Astyanax mexicanus. PLoS ONE, 7(11). https://doi.org/10.1371/journal.pone.0050308

Dumont, J. N. (1972). Oogenesis in Xenopus laevis (Daudin). I. Stages of oocyte development in laboratory maintained animals. Journal of Morphology, 136(2), 153-179. https://doi.org/10.1002/jmor.1051360203

Eagleson, G., Ferreiro, B., \& Harris, W. A. (1995). Fate of the anterior neural ridge and the morphogenesis of the xenopus forebrain. Journal of Neurobiology, 28(2), 146-158. https://doi.org/10.1002/neu.480280203

Eagleson, G. W., \& Dempewolf, R. D. (2002). The role of the anterior neural ridge and Fgf-8 in early forebrain patterning and regionalization in Xenopus laevis. Comparative Biochemistry and Physiology Part B, 132(1), 179-189.

Eberhart, J. K., Swartz, M. E., Crump, J. G., \& Kimmel, C. B. (2006). Early Hedgehog signaling from neural to oral epithelium organizes anterior craniofacial development. Development, 133(6), 1069-1077. https://doi.org/10.1242/dev.02281

Eiraku, M., Takata, N., Ishibashi, H., Kawada, M., Sakakura, E., Okuda, S., ... Sasai, Y. (2011). Self-organizing optic-cup morphogenesis in three-dimensional culture. Nature, 472, 51-56. https://doi.org/10.1038/nature09941

Ekker, S. C., Ungar, A. R., Greenstein, P., von Kessler, D. P., Porter, J. A., Moon, R. T., \& Beachy, P. A. (1995). Patterning activities of vertebrate hedgehog proteins in the developing eye and brain. Current Biology, 5(8), 944-955. https://doi.org/10.1016/S0960-9822(95)00185-0 
Elliott, K. L., Houston, D. W., \& Fritzsch, B. (2013). Transplantation of Xenopus laevis Tissues to Determine the Ability of Motor Neurons to Acquire a Novel Target. PLoS ONE, 8(2), e55541. https://doi.org/10.1371/journal.pone.0055541

Endo, T., Yokoyama, H., Tamura, K., \& Ide, H. (1997). Shh expression in developing and regenerating limb buds of Xenopus laevis. Developmental Dynamics, 209(2), $227-232$.

Fabbri, M., Mongiardino Koch, N., Pritchard, A. C., Hanson, M., Hoffman, E., Bever, G. S., ... Bhullar, B. A. S. (2017). The skull roof tracks the brain during the evolution and development of reptiles including birds. Nature Ecology and Evolution, 1(10), 1543-1550. https://doi.org/10.1038/s41559-017-0288-2

Falk, D. (1987). Hominid Paleoneurology. Annual Review of Anthropology, 16(1), 13-28. https://doi.org/10.1146/annurev.an.16.100187.000305

Fitz, C. R. (1994). Holoprosencephaly and septo-optic dysplasia. Neuroimaging Clinics of North America, 4(2), 263-281. Retrieved from https://europepmc.org/article/med/8081628

Gilbert, S. F. (2000). Development of the Vertebrate Eye. In Developmental Biology (6th ed.). Sunderland, MA: Sinauer Associates. Retrieved from https://www.ncbi.nlm.nih.gov/books/NBK10024/

Grenier, J., Teillet, M.-A., Grifone, R., Kelly, R. G., \& Duprez, D. (2009). Relationship between Neural Crest Cells and Cranial Mesoderm during Head Muscle Development. PLOS ONE, 4(2), e4381. https://doi.org/10.1371/JOURNAL.PONE.0004381

Gross, J. B., \& Hanken, J. (2008). Segmentation of the vertebrate skull: Neural-crest derivation of adult cartilages in the clawed frog, Xenopus laevis. Integrative and Comparative Biology, 48(5), 681-696. https://doi.org/10.1093/icb/icn077

Hanken, J., \& Gross, J. B. (2005). Evolution of cranial development and the role of neural crest: Insights from amphibians. Journal of Anatomy, 207(5), 437-446. https://doi.org/10.1111/j.1469-7580.2005.00481.x

Harland, R. M., \& Grainger, R. M. (2011). Xenopus research: Metamorphosed by genetics and genomics. Trends in Genetics, 27(12), 507-515. https://doi.org/10.1016/j.tig.2011.08.003

Heasman, J., Demel, T., Xanthos, J., Lohr, J., Sun, B., Sive, H., ... Heasman, J. (2006). Patterning the early Xenopus embryo. Development (Cambridge, England), 133(7), 1205-1217. https://doi.org/10.1242/dev.02304

Heavner, W., \& Pevny, L. (2012). Eye development and retinogenesis. Cold Spring Harbor Perspectives in Biology, 4(12). https://doi.org/10.1101/cshperspect.a008391 
Helms, J. A., Cordero, D., \& Tapadia, M. D. (2005, March 1). New insights into craniofacial morphogenesis. Development. The Company of Biologists. https://doi.org/10.1242/dev.01705

Herrera-Rincon, C., Pai, V. P., Moran, K. M., Lemire, J. M., \& Levin, M. (2017). The brain is required for normal muscle and nerve patterning during early Xenopus development. Nature Communications, 8(1), 1-18. https://doi.org/10.1038/s41467-017-00597-2

Hirasawa, T., \& Kuratani, S. (2015). Evolution of the vertebrate skeleton: morphology, embryology, and development. Zoological Letters, 1(1). https://doi.org/10.1186/s40851-014-0007-7

Hoffmeister, M., Krieg, J., Ehrke, A., Seigfried, F. A., Wischmann, L., Dietmann, P., ... Oess, S. (2017). Developmental neurogenesis in mouse and Xenopus is impaired in the absence of Nosip. Developmental Biology, 429(1), 200-212. https://doi.org/10.1016/J.YDBIO.2017.06.026

Hoffmeister, M., Prelle, C., Küchler, P., Kovacevic, I., Moser, M., Müller-Esterl, W., \& Oess, S. (2014). The Ubiquitin E3 Ligase NOSIP Modulates Protein Phosphatase 2A Activity in Craniofacial Development. PLOS ONE, 9(12), e116150. https://doi.org/10.1371/JOURNAL.PONE.0116150

Hofman, M. A. (2014). Evolution of the human brain: when bigger is better. Frontiers in Neuroanatomy, O(MAR), 15. https://doi.org/10.3389/FNANA.2014.00015

Houart, C., Westerfield, M., \& Wilson, S. W. (1998). A small population of anterior cells patterns the forebrain during zebrafish gastrulation. Nature, 391(6669), 788-792. https://doi.org/10.1038/35853

Hu, D., \& Marcucio, R. S. (2009). Unique organization of the frontonasal ectodermal zone in birds and mammals. Developmental Biology, 325(1), 200-210. https://doi.org/10.1016/j.ydbio.2008.10.026

Illes, J., Winterbottom, E., \& Isaacs, H. (2009). Cloning and expression analysis of the anterior parahox genes, Gsh1 and Gsh2 from Xenopus tropicalis.

Developmental Dynamics, 238(1), 194-203. https://doi.org/10.1002/DVDY.21816

Jiang, X., Iseki, S., Maxson, R. E., Sucov, H. M., \& Morriss-Kay, G. M. (2002). Tissue origins and interactions in the mammalian skull vault. Developmental Biology, 241(1), 106-116. https://doi.org/10.1006/dbio.2001.0487

Kalluri, R., \& Weinberg, R. A. (2009). The basics of epithelial-mesenchymal transition. The Journal of Clinical Investigation, 119(6), 1428. https://doi.org/10.1172/JCI39104

Kawahara, A., Baker, B. S., \& Tata, J. R. (1991). Developmental and regional 
expression of thyroid hormone receptor genes during Xenopus metamorphosis. Development, 112(4).

Kawasaki, K., \& Richtsmeier, J. T. (2017). Associations of the chondrocranium and dermatocranium. In C. J. Percival \& J. T. Richtsmeier (Eds.), Building Bones: bone development and formation in anthropology (pp. 52-78). Cambridge: Cambridge University Press.

Kha, C. X., Guerin, D. J., \& Ai-Sun Tseng, K. (2019). Using the Xenopus developmental eye regrowth system to distinguish the role of developmental versus regenerative mechanisms. Frontiers in Physiology, 10(MAY), 502. https://doi.org/10.3389/fphys.2019.00502

Kha, C. X., Son, P. H., Lauper, J., \& Tseng, K. A. S. (2018). A model for investigating developmental eye repair in Xenopus laevis. Experimental Eye Research, 169, 38-47. https://doi.org/10.1016/j.exer.2018.01.007

Kiecker, C., \& Lumsden, A. (2005). Compartments and their boundaries in vertebrate brain development. Nature Reviews Neuroscience, 6, 553-564. https://doi.org/10.1038/NRN1702

Kieny, M., Mauger, A., \& Sengel, P. (1972). Early regionalization of the somitic mesoderm as studied by the development of the axial skeleton of the chick embryo. Developmental Biology, 28, 142-161.

Klein, S. L., \& Graziadei, P. P. C. (1983). The differentiation of the olfactory placode in Xenopus laevis: A light and electron microscope study. Journal of Comparative Neurology, 217(1), 17-30.

Kobayashi, D., Kobayashi, M., Matsumoto, K., Ogura, T., Nakafuku, M., \& Shimamura, K. (2002). Early subdivisions in the neural plate define distinct competence for inductive signals. Development, 129(1), 83-93. https://doi.org/10.1242/DEV.129.1.83

Kuratani, S., Oisi, Y., \& Ota, K. G. (2016). Evolution of the Vertebrate Cranium: Viewed from Hagfish Developmental Studies. Https://Doi.Org/10.2108/Zs150187, 33(3), 229-238. https://doi.org/10.2108/ZS150187

Kuroda, H., Wessely, O., \& De Robertis, E. M. (2004). Neural induction in Xenopus: Requirement for ectodermal and endomesodermal signals via Chordin, Noggin, $\beta$-Catenin, and Cerberus. PLoS Biology, 2(5). https://doi.org/10.1371/journal.pbio.0020092

Lee-Liu, D., Méndez-Olivos, E. E., Muñoz, R., \& Larraín, J. (2017). The African clawed frog Xenopus laevis: A model organism to study regeneration of the central nervous system. Neuroscience Letters, 652, 82-93. https://doi.org/10.1016/J.NEULET.2016.09.054 
Lee, D. C., Hamm, L. M., \& Moritz, O. L. (2013). Xenopus laevis tadpoles can regenerate neural retina lost after physical excision but cannot regenerate photoreceptors lost through targeted ablation. Investigative Ophthalmology and Visual Science, 54(3), 1859-1867. https://doi.org/10.1167/iovs.12-10953

Lesciotto, K. M., \& Richtsmeier, J. T. (2019). Craniofacial skeletal response to encephalization: How do we know what we think we know? Am J Phys Anthropol, 168(67), 27-46. https://doi.org/10.1002/ajpa.23766

Lewis, W. H. (1904). Experimental studies on the development of the eye in amphibia. I. On the origin of the lens. Rana palustris. American Journal of Anatomy, 3(4), 505-536. https://doi.org/10.1002/aja.1000030405

Liem, J., Jessell, T. M., \& Briscoe, J. (2000). Regulation of the neural patterning activity of sonic hedgehog by secreted BMP inhibitors expressed by notochord and somites. Development, 127(22), 4855-4866.

https://doi.org/10.1242/dev.127.22.4855

Lukas, P., \& Olsson, L. (2018). Sequence and timing of early cranial skeletal development in Xenopus laevis. Journal of Morphology, 279(1), 62-74. https://doi.org/10.1002/jmor.20754

Maddin, H. C., Piekarski, N., Sefton, E. M., \& Hanken, J. (2016). Homology of the cranial vault in birds: New insights based on embryonic fate-mapping and character analysis. Royal Society Open Science, 3(8).

https://doi.org/10.1098/rsos.160356

Marcucio, R. S., Cordero, D. R., Hu, D., \& Helms, J. A. (2005). Molecular interactions coordinating the development of the forebrain and face.

Developmental Biology, 284(1), 48-61. https://doi.org/10.1016/J.YDBIO.2005.04.030

Mariani, F. V, Ahn, C. P., \& Martin, G. R. (2008). Genetic evidence that FGFs have an instructive role in limb proximal-distal patterning. Nature, 453(7193), 401405. https://doi.org/10.1038/nature06876

Markey, M. J., \& Marshall, C. R. (2007). Terrestrial-style feeding in a very early aquatic tetrapod is supported by evidence from experimental analysis of suture morphology. PNAS, 104(17), 7134-7138. https://doi.org/10.1073/pnas.0701706104

McBratney-Owen, B., Iseki, S., Bamforth, S. D., Olsen, B. R., \& Morriss-Kay, G. M. (2008). Development and tissue origins of the mammalian cranial base.

Developmental Biology, 322(1), 121-132. https://doi.org/10.1016/j.ydbio.2008.07.016

Meyer, R. (1913). Die ursächlichen Beziehungen zwischen dem Situs viscerum und 
Situs cordis. Arch. Entw. Mech., 37, 85-107.

Millet, S., Campbell, K., Epstein, D. J., Losos, K., Harris, E., \& Joyner, A. L. (1999). A role for $\mathrm{Gbx} 2$ in repression of Otx 2 and positioning the mid/hindbrain organizer. Nature, 401(6749), 161-164. https://doi.org/10.1038/43664

Moreno, N., Rétaux, S., \& González, A. (2008). Spatio-temporal expression of Pax6 in Xenopus forebrain. Brain Research, 1239, 92-99.

https://doi.org/10.1016/J.BRAINRES.2008.08.052

Morriss-Kay, G. M. (2001). Derivation of the mammalian skull vault. J. Anat, 199, $143-151$.

Müller, F., \& O’Rahilly, R. (1991). Development of anencephaly and its variants. American Journal of Anatomy, 190(3), 193-218.

https://doi.org/10.1002/aja.1001900302

Muzio, L., \& Mallamaci, A. (2003). Emx1, Emx2 and Pax6 in specification, regionalization and arealization of the cerebral cortex. Cerebral Cortex, 13(6), 641-647. https://doi.org/10.1093/cercor/13.6.641

Naumann, B., \& Olsson, L. (2018). Three-dimensional reconstruction of the cranial and anterior spinal nerves in early tadpoles of Xenopus laevis (Pipidae, Anura). Journal of Comparative Neurology, 526(5), 836-857.

https://doi.org/10.1002/CNE.24370

Nieuwkoop, P. D., \& Faber, J. (1994). Normal Table of Xenopus Laevis (Daudin). New York: Garland Publishing Inc.

Noden, D. M. (1983). The role of the neural crest in patterning of avian cranial skeletal, connective, and muscle tissues. Developmental Biology, 96(1), 144165 .

Noden, D. M., \& Trainor, P. A. (2005). Relations and interactions between cranial mesoderm and neural crest populations. Journal of Anatomy. Blackwell Publishing Ltd. https://doi.org/10.1111/j.1469-7580.2005.00473.x

Ohkubo, Y., Chiang, C., \& Rubenstein, J. L. R. (2002). Coordinate regulation and synergistic actions of BMP4, SHH and FGF8 in the rostral prosencephalon regulate morphogenesis of the telencephalic and optic vesicles. Neuroscience, 111(1), 1-17. https://doi.org/10.1016/S0306-4522(01)00616-9

Paganelli, A., Gnazzo, V., Acosta, H., López, S. L., \& Carrasco, A. E. (2010). Glyphosate-based herbicides produce teratogenic effects on vertebrates by impairing retinoic acid signaling. Chemical Research in Toxicology, 23(10), 1586-1595. https://doi.org/10.1021/tx1001749

Peyrot, S. M., Wallingford, J. B., \& Harland, R. M. (2011). A revised model of Xenopus dorsal midline development: Differential and separable requirements 
for Notch and Shh signaling. Developmental Biology, 352(2), 254-266. https://doi.org/10.1016/J.YDBIO.2011.01.021

Piekarski, N., Gross, J. B., \& Hanken, J. (2014). Evolutionary innovation and conservation in the embryonic derivation of the vertebrate skull. Nature Communications, 5(1), 1-9. https://doi.org/10.1038/ncomms6661

Pitirri, M. K., Kawasaki, K., \& Richtsmeier, J. T. (2020). It takes two: Building the vertebrate skull from chondrocranium and dermatocranium. Vertebrate Zoology, 70(4), 600. Retrieved from /pmc/articles/PMC7644101/

Pownall, M. E., \& Isaacs, H. V. (2010). FGF Signalling in Vertebrate Development. FGF Signalling in Vertebrate Development. San Rafael: Morgan \& Claypool Life Sciences.

Pressler, K. (1911). Beobachtungen und Versuche über den normalen und inversen Situs viscerum und cordis bei Anuranlarven. Arch. Entw. Mech., 32.

Quinzio, S. I., \& Fabrezi, M. (2019). The peripheral nerves of Lepidobatrachus tadpoles (Anura, Ceratophryidae). Journal of Morphology, 280(1), 4-19. https://doi.org/10.1002/jmor.20849

Raam, M. S., Solomon, B. D., Shalev, S. A., \& Muenke, M. (2010, February 15). Holoprosencephaly and craniosynostosis: A report of two siblings and review of the literature. American Journal of Medical Genetics, Part C: Seminars in Medical Genetics. NIH Public Access. https://doi.org/10.1002/ajmg.c.30234

Richman, J. M., \& Tickle, C. (1992). Epithelial-mesenchymal interactions in the outgrowth of limb buds and facial primordia in chick embryos. Developmental Biology, 154(2), 299-308.

Richtsmeier, J. T., \& Flaherty, K. (2013, April 23). Hand in glove: Brain and skull in development and dysmorphogenesis. Acta Neuropathologica. Springer. https://doi.org/10.1007/s00401-013-1104-y

Roček, Z. (1988). Origin and evolution of the frontoparietal complex in anurans. Amphibia-Reptilia, 9, 385-403. Retrieved from http://rocek.gli.cas.cz/reprints/frpcomplex.pdf

Roelink, H., Augsburger, A., Heemskerk, J., Korzh, V., Norlin, S., Altaba, A. R. i, ... Dodd, J. (1994). Floor plate and motor neuron induction by vhh-1, a vertebrate homolog of hedgehog expressed by the notochord. Cell, 76(4), 761-775.

Roessler, E., Belloni, E., Gaudenz, K., Vargas, F., Scherer, S. W., Tsui, L. C., \& Muenke, M. (1997). Mutations in the C-terminal domain of Sonic Hedgehog cause holoprosencephaly. Human Molecular Genetics, 6(11), 1847-1853. https://doi.org/10.1093/hmg/6.11.1847 
Romer, A. S., \& Edinger, T. (1942). Endocranial casts and brains of living and fossil amphibia. The Journal of Comparative Neurology, 77(2), 355-389.

https://doi.org/10.1002/cne.900770203

Romer, A. S., \& Parsons, T. S. (1977). The Vertebrate Body (5th ed.). Philadelphia: W. B. Saunders Co.

Schlosser, G., \& Ahrens, K. (2004). Molecular anatomy of placode development in Xenopus laevis. Developmental Biology, 271(2), 439-466.

https://doi.org/10.1016/j.ydbio.2004.04.013

Schlosser, G., \& Glenn Northcutt, R. (2000). Development of Neurogenic Placodes in Xenopus laevis. J. Comp. Neurol, 418(2), 121-146.

Schmidt, L., Taiyab, A., Melvin, V. S., Jones, K. L., \& Williams, T. (2018). Increased FGF8 signaling promotes chondrogenic rather than osteogenic development in the embryonic skull. Disease Models \& Mechanisms, 11(6). https://doi.org/10.1242/DMM.031526

Schoch, R. R. (2014). Amphibian skull evolution: The developmental and functional context of simplification, bone loss and heterotopy. Journal of Experimental Zoology Part B: Molecular and Developmental Evolution, 322(8), 619-630. https://doi.org/10.1002/jez.b.22599

Schwanzel-Fukuda, M., \& Pfaff, D. W. (1989). Origin of luteinizing hormonereleasing hormone neurons. Nature, 338(6211), 161-164.

https://doi.org/10.1038/338161a0

Sedra, S. N., \& Michael, M. I. (1957). The development of the skull, visceral arches, larynx and visceral muscles of the South African clawed toad, Xenopus laevis during the process of metamorphosis (from Stage 55 to stage 66). Amsterdam: N.V. Noord-Hollandsche Uitgevers Maatschappij.

Senevirathne, G., Kerney, R., \& Meegaskumbura, M. (2017). Comparative Postembryonic Skeletal Ontogeny in Two Sister Lineages of Old World Tree Frogs (Rhacophoridae: Taruga, Polypedates). PLOS ONE, 12(1), e0167939. https://doi.org/10.1371/JOURNAL.PONE.0167939

Session, A. M., Uno, Y., Kwon, T., Chapman, J. A., Toyoda, A., Takahashi, S., ... Rokhsar, D. S. (2016). Genome evolution in the allotetraploid frog Xenopus laevis. Nature, 538(7625), 336-343. https://doi.org/10.1038/nature19840

Shim, K. W., Park, E. K., Kim, J. S., Kim, Y. O., \& Kim, D. S. (2016).

Neurodevelopmental problems in non-syndromic craniosynostosis. Journal of Korean Neurosurgical Society, 59(3), 242-246. https://doi.org/10.3340/jkns.2016.59.3.242

Silver, D. L., Hou, L., \& Pavan, W. J. (2006). The genetic regulation of pigment cell 
development. Advances in Experimental Medicine and Biology. Springer New York. https://doi.org/10.1007/978-0-387-46954-6_9

Slater, B. J., Liu, K. J., Kwan, M. D., Quarto, N., \& Longaker, M. T. (2009). Cranial Osteogenesis and Suture Morphology in Xenopus laevis: A Unique Model System for Studying Craniofacial Development. PLoS ONE, 4(1), e3914. https://doi.org/10.1371/journal.pone.0003914

Spemann, H. (1906). Über embryonale Transplantation. Verh. Dtsch. Zool. Ges., 16, 195-202.

Streit, A. (2007). The preplacodal region: An ectodermal domain with multipotential progenitors that contribute to sense organs and cranial sensory ganglia. International Journal of Developmental Biology, 51(6-7), 447-461. https://doi.org/10.1387/ijdb.072327as

Tandon, P., Conlon, F., Furlow, J. D., \& Horb, M. E. (2017). Expanding the genetic toolkit in Xenopus: Approaches and Opportunities for Human Disease Modeling. Developmental Biology, 426(2), 325. https://doi.org/10.1016/J.YDBIO.2016.04.009

Thompson, H., Ohazama, A., Sharpe, P. T., \& Tucker, A. S. (2012). The origin of the stapes and relationship to the otic capsule and oval window. Developmental Dynamics, 241(9), 1396-1404. https://doi.org/10.1002/DVDY.23831

Trueb, L., \& Hanken, J. (1992). Skeletal development in Xenopus laevis (Anura: Pipidae). Journal of Morphology, 214(1), 1-41. https://doi.org/10.1002/jmor.1052140102

Trumpp, A., Depew, M. J., Rubenstein, J. L. R., Bishop, J. M., \& Martin, G. R. (1999). Cre-mediated gene inactivation demonstrates that FGF8 is required for cell survival and patterning of the first branchial arch. Genes and Development, 13(23), 3136-3148. https://doi.org/10.1101/gad.13.23.3136

Tsien, J., Chen, D., Gerber, D., Tom, C., Mercer, E., Anderson, D., Mayford, M., Kandel, E., \& Tonegawa, S. (1996). Subregion- and cell type-restricted gene knockout in mouse brain. Cell, 87(7), 1317-1326. https://doi.org/10.1016/S00928674(00)81826-7

van Ginneken, V., van Meerveld, A., Wijgerde, T., Verheij, E., de Vries, E., \& van der Greef, J. (2017). Hunter-prey correlation between migration routes of African buffaloes and early hominids: Evidence for the "Out of Africa" hypothesis. Integrative Molecular Medicine, 4(3), 1-5. https://doi.org/10.15761/IMM.1000287

Vega-Lopez, G. A., Cerrizuela, S., Tribulo, C., Aybar, M. J., \& Barbieri, F. D. (2018). Neurocristopathies: New insights 150 years after the neural crest discovery. Developmental Biology, 444, S110-S143. 
https://doi.org/10.1016/j.ydbio.2018.05.013

Vieira, C., Pombero, A., García-Lopez, R., Gimeno, L., Echevarria, D., \& Martínez, S. (2010). Molecular mechanisms controlling brain development: An overview of neuroepithelial secondary organizers. International Journal of Developmental Biology, 54(1), 7-20. https://doi.org/10.1387/ijdb.092853cv

Werneburg, I. (2019). Morphofunctional Categories and Ontogenetic Origin of Temporal Skull Openings in Amniotes. Frontiers in Earth Science, 7, 13. https://doi.org/10.3389/feart.2019.00013

Wilson, S. W., \& Houart, C. (2004). Early steps in the development of the forebrain. Developmental Cell, 6(2), 167-181. https://doi.org/10.1016/S15345807(04)00027-9

Wray, S., Grant, P., \& Gainer, H. (1989). Evidence that cells expressing luteinizing hormone-releasing hormone mRNA in the mouse are derived from progenitor cells in the olfactory placode (prenatal development/in situ hybridization/histochenustry/immunocytochemistry/[3H]thymidine autoradiography). Proceedings of the National Academy of Sciences of the United States of America, 86(20), 8132-8136.

Yang, J., Aguero, T., \& King, M. Lou. (2015). The Xenopus Maternal-to-Zygotic Transition from the Perspective of the Germline. Current Topics in Developmental Biology, 113, 271-303. https://doi.org/10.1016/bs.ctdb.2015.07.021

Yang, Y., \& Niswander, L. (1995). Interaction between the signaling molecules WNT7a and SHH during vertebrate limb development: dorsal signals regulate anteroposterior patterning. Cell, 80(6), 939-947. https://doi.org/10.1016/00928674(95)90297-X

Zahn, N., Levin, M., \& Adams, D. S. (2017). The Zahn drawings: New illustrations of Xenopus embryo and tadpole stages for studies of craniofacial development.

Development (Cambridge), 144(15), 2708-2713. https://doi.org/10.1242/dev.151308

Zeltser, L. M., Larsen, C. W., \& Lumsden, A. (2001). A new developmental compartment in the forebrain regulated by Lunatic fringe. Nature Neuroscience, 4(7), 683-684. https://doi.org/10.1038/89455

Ziermann, J. M., \& Olsson, L. (2007). Patterns of spatial and temporal cranial muscle development in the African clawed frog, Xenopus laevis (Anura: Pipidae). Journal of Morphology, 268(9), 791-804. https://doi.org/10.1002/jmor.10552 